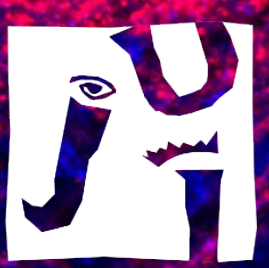

UNIVERSITAT

JAUME-1

Unravelling the role of the cerebellum in drug addiction. Cerebellum-prefrontal networks in drug-induced preference memory

Author:

Isis Gil Miravet

Directed by:

Dra. Marta Miquel Salgado-Araujo 



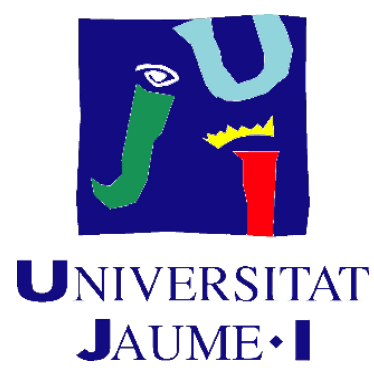

Programa de Doctorat en Psicologia

Escola de Doctorat de la Universitat Jaume I

Unravelling the role of the cerebellum in drug addiction. Cerebellum-prefrontal networks in drug-induced preference memory

Memòria presentada per Isis Gil Miravet per a optar al grau de doctora per la Universitat Jaume I

Isis Gil Miravet

Marta Miquel Salgado-Araujo 



\section{Finançament rebut}

Aquesta recerca ha comptat amb el suport de les següents beques i subvencions publiques:

- Universitat Jaume I (PREDOC2014/11)

- Universitat Jaume I (E-2016-44)

- Universitat Jaume I (14I307.01/1)

- Ministerio de Educación Cultura y Deporte (FPU12/04059)

- Ministerio de Economía y Competitividad (MINECO) (PSI2015-68600-P)

- Plan Nacional de Drogas 2017 (PND-132400) 

A la meua família 



\section{TABLE OF CONTENTS}

PREFACE

GENERAL INTRODUCTION

Addiction and drug reward

The addiction circuitry

Cerebellum: "the little brain" 9

Cerebellum anatomy 9

Cerebellum-cerebro reciprocal loops

The cerebellum in addiction

Drug-induced plasticity changes in the cerebellum

Chapter 1: The role of the cerebellum in drug-cue associative memory: functional interactions with the medial prefrontal cortex

Chapter 2: Changes in neural activity and perineuronal net expression in the cerebellum after deactivation of the medial prefrontal cortex

Chapter 3: From back to front: A functional model for the cerebellar modulation in the establishment of conditioned preferences for cocaine-related cues

GENERAL DISCUSSION

STRENGTHS AND PITFALLS

Strengths

Pitfalls, weaknesses, and future directions 

PREFACE 



\section{PREFACE}

The cerebellum has been "the long forgotten" in the addiction field for many years. Its study was limited to motor functions and underestimated in the rest of the brain functions. Fortunately, it has changed in the last decades, and the study of the cerebellum has been included in research on language, memory, emotions, decisionmaking, social behaviour, and drug addiction (Adamaszek et al, 2017; Blackwood et al, 2004; Broche-Pérez et al, 2016; Carbo-Gas et al, 2014a, 2014b, 2017; Carta et al, 2019; Courchesne and Allen, 1997; Mariën et al, 2014; Miquel et al, 2016; Moers-Hornikx et al, 2009; Moulton et al, 2014; Sacchetti et al, 2002a, 2004; Strata et al, 2011; Turner et al, 2007; Vazquez-Sanroman et al, 2015b).

Research on the cerebellum's role in addiction is the core of this doctoral thesis. Previous studies in our laboratory involved this structure in drug-induced preference conditioning (Carbo-Gas et al, 2014a, 2014b, 2017). This research demonstrated two cerebellar hallmarks of preference for cues linked to the cocaine experience. Both an increased cFos expression and stronger fully condensed perineuronal nets (PNNs) in the apical region of the granule cell layer (the dorsal region of the cerebellar cortex) were observed only when animals expressed a preference towards an odour associated with the drug. These distinctive features were not seen if the animals did not exhibit cocainerelated memory. These findings supported and extended the results of neuroimaging studies on cue-reactivity in addicted cohorts, which found increased greater activation in the cerebellum after the presentation of drug-related cues (Bonson et al, 2002; Grant et al, 1996; Schneider et al, 2001).

Overall, these earlier results suggested that the establishment or expression of cocaineinduced conditioned memories somehow entails cerebellar activation. Moreover, they indicated that cocaine-induced memory encourages, at the cerebellar level, one of the mechanisms for synaptic stabilization, the expression of PNNs. In that way, the cerebellum might be part of the functional networks that represent long-lasting drugrelated memories (Sorg et al, 2016). Therefore, this doctoral thesis intends to be the first attempt to propose a causative working model for the cerebellum's role in druginduced memories. 
In the following pages, we managed to explore the role of the cerebellum in cocaineinduced conditioned preference and its functional and anatomical relationships with the medial prefrontal cortex. We addressed this goal using temporal or permanent brain deactivations.

The present doctoral dissertation begins with a small theoretical introduction about addiction, followed by a description of the cerebellar anatomy, and a summary of the state of the art in the field.

The first chapter entitled "The role of the cerebellum in drug-cue associative memory: functional interactions with the medial prefrontal cortex" is an article already published in the European Journal of Neuroscience in which we investigated the role that specific regions of the cerebellum and medial prefrontal cortex (mPFC) play in the acquisition of cocaine-induced preference conditioning. To the best of our knowledge, this is the first study focused on the causative role of the cerebellum in drug addiction. Importantly, the results indicated that the deactivation of these two distal regions generates similar effects on cocaine-related behaviour. Nevertheless, the effects would depend on the specific prefrontal and cerebellar regions deactivated. This first study revealed a close interaction, probably compensatory, between the dorsal cerebellum and the infralimbic cortex in the establishment of cocaine-related memory.

To ascertain the nature of cerebellar-prefrontal relationships and to propose a working functional model for cerebellar-prefrontal interactions, we investigated the consequences of impairing the function in one region for activity and plasticity in the other region. Thus, in the second chapter, "Changes in neural activity and perineuronal net expression in the cerebellum after deactivation of the medial prefrontal cortex" we addressed an exhaustive analysis of cFos and PNN expression in the cerebellum and other cerebral regions after mPFC deactivations. Importantly, cerebellar activity and PNN expression increased only after infralimbic deactivation. Based on a more detailed analysis, we discussed whether the observed functional interaction could be seen or not as compensatory.

In the third chapter, "From back to front: A functional model for the cerebellar modulation in the establishment of conditioned preferences for cocaine-related cues", 
we explored the activity and plasticity in the striatum and mPFC after the cerebellar impairment. Additionally, we accomplished a tracing study using anterograde and retrograde tracers in order to build a working neuroanatomical model to explain the facilitative effect of the cerebellar lesion on cocaine-induced conditioned memory. Our findings hinted at an inhibitory control of the posterior vermis over the striatum and MPFC through the VTA.

Finally, the doctoral thesis concludes with a general discussion, which helps to explain the results of the three chapters, and presents a hypothetical model to encourage future research. We also discuss the strengths and pitfalls of our findings. References can be found at the end of each chapter and on the last pages, after the strengths and pitfalls section. 


$$
4
$$


GENERAL INTRODUCTION 



\section{GENERAL INTRODUCTION}

\section{Addiction and drug reward}

Drug addiction is a disorder of the Central Nervous System (CNS) whose main symptoms have been characterized by escalating drug use, loss of control over limiting drug intake, the emergence of chronic compulsive drug-seeking, and a marked reduction of interest in other objectives and rewards (Robinson and Berridge, 2003). Initially, drug reward is the main premise for drug consumption in both humans and animals (Wise, 2009). Thus, addictive drugs compete with other natural stimuli in terms of their ability to initiate goal-directed behaviours, and trigger adaptation and neuroplasticity processes. However, drugs of abuse progressively reduce the ability of individuals to initiate and sustain actions towards natural stimuli (Hyman et al, 2006; Kalivas and Volkow, 2005). Although much of the initial studies of drug addiction focused on the critical impact of addictive substances on the CNS, now attention is being directed towards the effects of chronic drug-intake and long-term neuronal brain changes that end in a relapse. A major factor for relapse is the persistence of maladaptive drug-associated memories, which can preserve drug-seeking and taking behaviour. In this respect, there is increasing evidence to support the ability of addictive drugs to promote stable changes in synaptic connections of brain circuits responsible for memories and behaviours established by Pavlovian and instrumental conditioning (Everitt and Robbins, 2005). Indeed, drug addiction results from an aberrant learning that induces the formation of strong instrumental memories, linking actions to drug-seeking and taking outcomes that are finally expressed as persistent stimulus-response habits (Milton and Everitt, 2012). Initially, neutral environmental stimuli become associated with drug highs through Pavlovian conditioning, driving the subsequent interactions between Pavlovian and instrumental memories to influence relapse. Therefore, long-lasting drug-induced modifications in Pavlovian and instrumental learning appear to be the most explanatory mechanism for the establishment of drug addiction.

Everitt et al. (2001) showed that the aberrant engagement of Pavlovian and instrumental learning mechanisms leads to enhanced learning about the actions and environmental drug-associated cues or conditioned stimuli (CSs) that predict 
opportunities for drug self-administration. They argued that these processes could be produced by the ability of drugs of abuse to increase the release of dopamine in several regions of the striatum-cortico-limbic system. Consequently, repeated drug-taking indeed these CSs to acquire an increasing role in controlling drug-seeking behaviour. As the state of addiction develops, the previously drug-paired CSs induced drug-seeking, independently of goal-directed actions. In consequence, drug consumption becomes controlled by stimulus-response mechanisms that are habitual and automatic, via CSinduced activation of drug-seeking motor programs in the dorsal striatum (Belin et al, 2009; Belin and Everitt, 2008). In some individuals, drug-seeking becomes compulsive and persistent and generates an insensitivity to devaluation or punishment (Ahmed, 2012; Belin and Everitt, 2008; Pelloux et al, 2007). In this way, the repeated association of a conditioned stimulus with drug effects seems to promote a shift from goal-directed instrumental behaviour to compulsive habits.

\section{The addiction circuitry}

Drug-induced memories are mainly stored through molecular interactions between dopaminergic and glutamatergic systems in the prefrontal-limbic-striatal networks (Everitt and Robbins, 2005).

The medial prefrontal cortex (MPFC) is responsible for executive control, which includes the representation of contingencies, representation of outcomes and their value, and

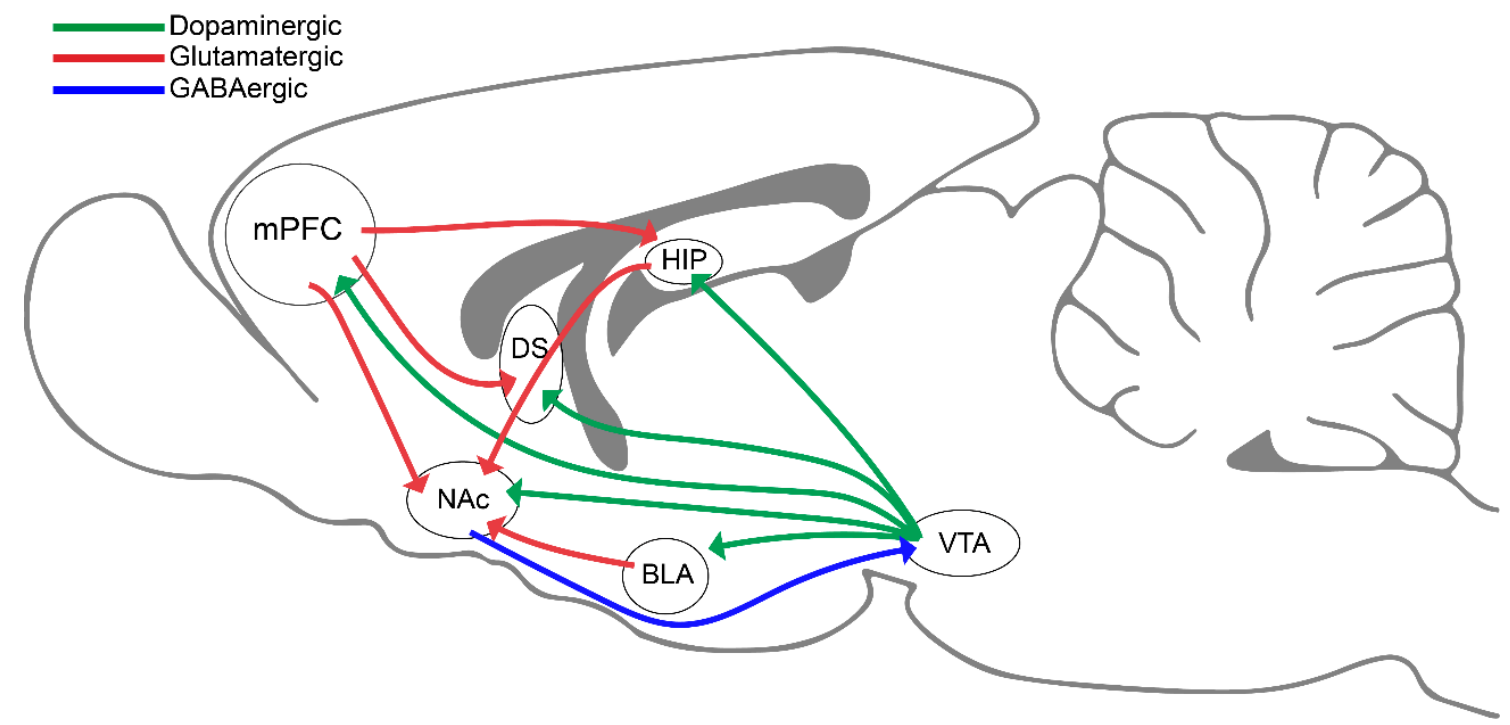

Figure 1. The addiction circuitry. Medial prefrontal cortex (mPFC), dorsolateral straitum (DL), nucleus accumbens (NAC), hippocampus (HIP), basolateral amygdala (BLA), ventral tegmental area (VTA). 
subjective states associated with drugs (Everitt and Robbins, 2005). The prefrontal cortex, along with the nucleus accumbens (NAc) and the dorsomedial striatum (DMS), orchestrate goal-directed actions, whereas the interactions between some regions in the MPFC and the dorsolateral striatum (DLS) underpin the establishment of habits (Smith and Graybiel, 2013). The reestablishment of drug-seeking after abstinence depends on the MPFC release of glutamate and dopamine in the nucleus accumbens core (NACC) and the integrity of the ventral pallidum (McFarland et al, 2003). The NAC exhibits neuroadaptations following drug experience, particularly changes in glutamatergic signalling (Kalivas and McFarland, 2003) that are hypothesized to increase the salience of drug-associated CSs and thereby their impact on behaviour (Kalivas, 2004). Additionally, long-lasting plasticity changes in the orbitofrontal cortex (OFC), basolateral amygdala (BLA) and $\mathrm{NACC}$, regions richly interconnected, underlying the capacity of conditioned reinforcers to encourage drug-seeking after long periods of abstinence (Kalivas and McFarland, 2003; Schoenbaum et al, 2003; Shaham et al, 2003). Dopamine and glutamate interactions in the BLA and NACC have also been observed in relapse (McFarland et al, 2003; See et al, 2001). Moreover, the BLA has been shown to be required for sensory-specific conditioned reinforcement and Pavlovian-instrumental transfer (Cardinal et al, 2002; Pelloux et al, 2013; Stefanik and Kalivas, 2013). The sequential phases of Pavlovian and instrumental learning may be especially relevant for the transition from initial drug use to drug abuse, and finally to compulsive drug-seeking. Pavlovian-instrumental transfer resulting in drug-seeking depends on afferents from specific regions of the mPFC to the DMS (Ostlund and Balleine, 2005; Yin et al, 2005). Likewise, the output from the NAcSh can influence the functioning of ascending dopamine projections to the $\mathrm{NACC}$, and from the $\mathrm{NACC}$ via the substantia nigra to other domains of the DMS (Haber et al, 2000). Thus, the potentiation of conditioned reinforcement by stimulant drugs and Pavlovian-instrumental transfer could result from drug's impact on the NAcSh, influencing processing of CSs in the NAcC and DMS.

The amygdala and hippocampus are both key components for mediating the ability of drug-related contexts to trigger drug-seeking and relapse (Bonson et al, 2002; Grant et al, 1996). Context-induced reinstatement of drug-seeking requires the hippocampus (Carballo-Márquez et al, 2009; Sierra-Mercado et al, 2011), similar to its role in 
contextual fear conditioning (Phillips and LeDoux, 1992). It has been observed that contexts previously associated with drug use can promote relapse in animals with an extensive history of drug self-administration (Bossert et al, 2011; Crombag and Shaham, 2002; Fuchs et al, 2005). In this way, the hippocampus would represent context as a CS, with the amygdala associating the hippocampal-encoded context with the affective value of the unconditioned stimulus. This is the case for contextual memories associated with aversive outcomes, as well as appetitive conditioning for natural and drug reinforcers (Hitchcott and Phillips, 1997; Matus-Amat et al, 2007).

In summary, plasticity mechanisms within the hippocampus, amygdala, and mPFC may all influence drug-seeking through their convergent projections to the NAc, perhaps competing for access to response strategies involving different prefrontal-striatal-limbic networks (Goto and Grace, 2005). It has been hypothesised that the transition from voluntary actions to more habitual modes of responding in drug-seeking involves a transfer from prefrontal to striatal control over responding, as well as from ventral to more dorsal striatal regions (Belin and Everitt, 2008; Everitt and Robbins, 2005), through a progressive recruitment of dopaminergic neurons in the midbrain (Haber et al, 2000). Together with an overreliance on striatal mechanisms, the progressive loss of control over drug consumption requires a reduction in the inhibitory control exercised by the prefrontal cortex (Volkow et al, 2013). Therefore, drug-seeking becomes less dependent on voluntary control and more prone to be triggered automatically and compulsively (Everitt and Robbins, 2005).

Beyond the traditional neuroanatomical model of drug addiction, an increasing amount of data suggests the involvement of the cerebellum in many of the affected brain functions in addicts (Miquel et al, 2009, 2016; Moulton et al, 2014). The research we present and discuss in this thesis strongly supports the inclusion of the cerebellum as a part of the circuit responsible for long-lasting drug-induced behavioural effects. 


\section{Cerebellum: "the little brain"}

\section{Cerebellum anatomy}

The cerebellum controls and regulates motor and non-motor functions. It is integrated by the vermis (the central region), the paravermal area (on each side of the vermis), and the hemispheres, which are the two most external regions. Two fissures divide the cerebellum in a rostral-caudal direction into anterior (lobules I to V), posterior (lobules VI to IX), and flocculonodular (lobule X) lobules (Marr, 1969). The cerebellum architecture consists of a thin layer of white matter covered by grey matter and three pair of deep cerebellar nuclei (medial/fastigial, interpositus, and lateral/dentate). The grey matter is composed by three layers: the molecular layer, Purkinje layer, and granular layer (Brodal, 2016; Voogd and Glickstein, 1998). The molecular layer is the outermost layer and contains few neuronal somas (basket and stellate cells), being mainly formed by dendrites and axons of other cells (Voogd and Glickstein, 1998). This layer includes dendritic arborizations of Purkinje cells, whose cell bodies are the predominant component of the Purkinje layer (Haines and Dietrichs, 2011). The granular layer is the deepest layer and limits with white matter. This layer is formed by Golgi, Lugaro, and unipolar brush cells, as well as in greater numbers, granule cells (Eccles et al, 1964). The majority of cells in the cerebellar cortex are glutamatergic neurons, including granule cells and unipolar brush cells. The rest of them are GABAergic neurons (Apps and Garwicz, 2005).

The cerebellar cortex receives information mainly from cerebral cortices, limbic areas and basal ganglia through two glutamatergic afferents. Climbing fibres, coming from the
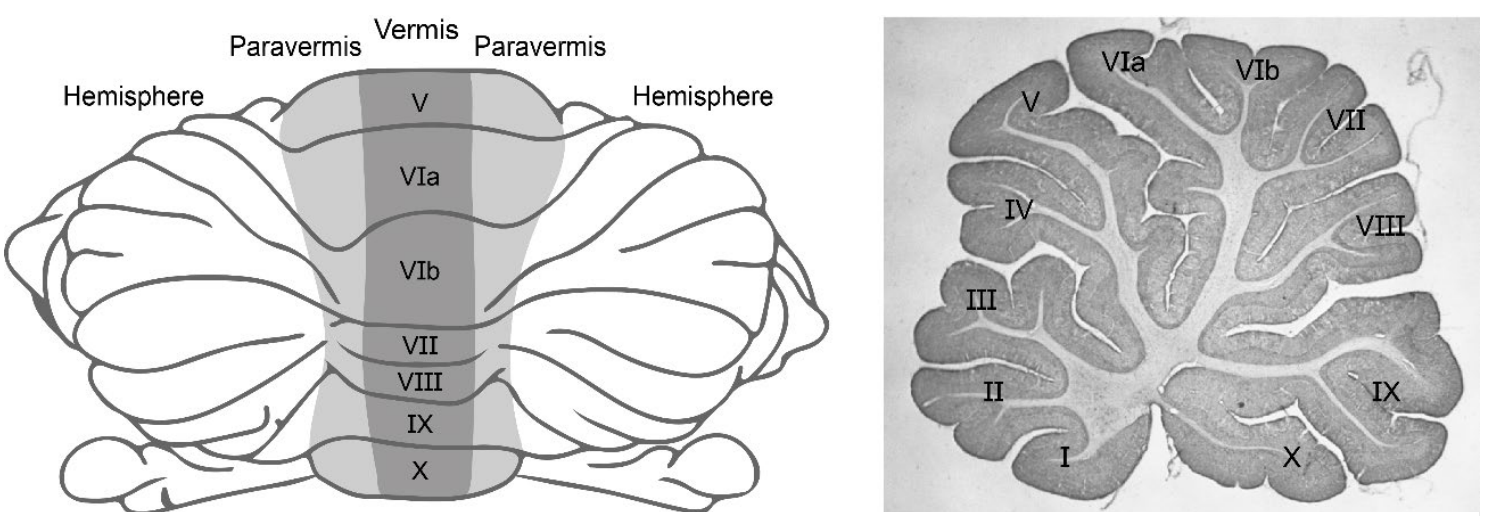

Figure 2. Cerebellum divisions. The posterior coronal image shows the vermis, paravermis and hemispheres, and the posterior lobules. The sagittal image shows a medial section and the distribution of all the lobules. 
inferior olive, climb up to dendrites of Purkinje neurons. Mossy fibres, originated in the pontine nuclei, synapse within the glomerulus, that included Golgi and granule cells (Albus, 1971; Andersen et al, 1992; Gilbert and Thach, 1977; Marr, 1969). The glomerulus is a structure wrapped into a glial lamina that limits the diffusion of the neurotransmitter (Barbour and Häusser,

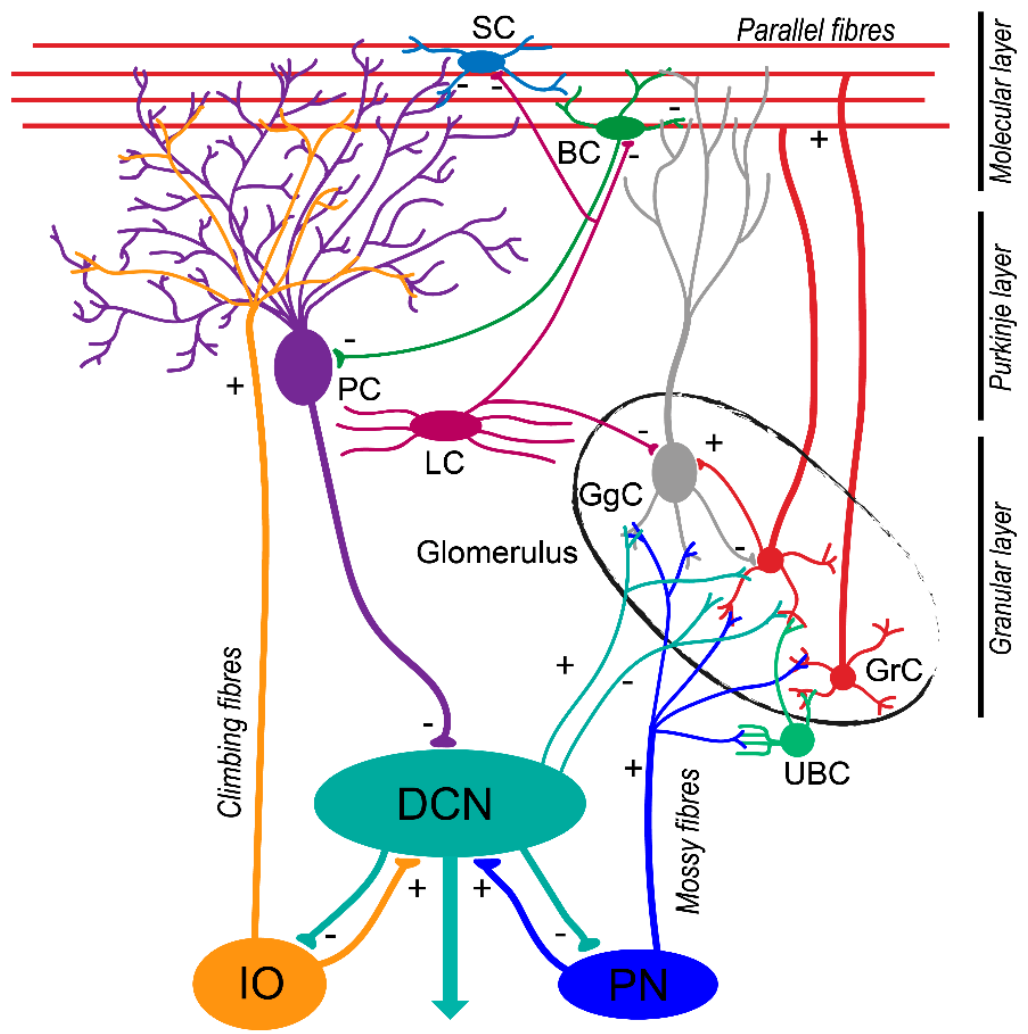

Figure 3. Cerebellar cortex. Stellate cell (SC), basket cell (BC), Purkinje cell $(P C)$, Lugar cell $(L C)$, unipolar brush cells $(U B C)$, Golgi cell $(G g C)$, granule cell $(\mathrm{GrC})$, deep cerebellar nuclei (DCN), inferior olive (IO), pontine nuclei $(P N)$.

1997). Golgi cells are the most numerous inhibitory interneuron of the granular layer and control the activity of as many as 100 billion granule cells (Marr, 1969). Golgi cells can reside at different depths in the granular layer, and their basal dendrites usually remain in the granular layer, while apical dendrites ascend into the molecular layer traversing the parallel fibre (Albus, 1971). The axons of granule cells ascend to the molecular layer and branch into parallel excitatory fibres that come into contact with Purkinje somas and dendrites (Brunel et al, 2004; Marr, 1969). Basket and stellate cells (inhibitory interneurons) regulate the excitatory inputs on Purkinje cells, and send inhibitory signals to the deep cerebellar nuclei (DCN), which control the final output of the cerebellum (Ito, 1984; White and Sillitoe, 2013). Golgi cells also receive inhibitory innervation from stellate and basket cells, as well as Lugaro cells (Eccles et al, 1964). Moreover, DCN receive axon collaterals from the inferior olive and pontine nuclei. The DCN are the main output from the cerebellum and are composed mainly of neurons with large glutamatergic projections, although they harbour a small number of GABAergic neurons that project towards the inferior olivary and pontine nuclei (Gao et al, 2016; 
Voogd and Glickstein, 1998). The activation of these glutamatergic neurons releases the information out of the cerebellum (Shinoda et al, 2000).

\section{Cerebellum-cerebro reciprocal loops}

Several anatomical and functional studies have proposed that the cerebellum and the striatum-cortico-limbic circuit are interconnected (Bostan et al, 2013; Bostan and Strick, 2018; Rogers et al, 2011). Bostan et al. (2010) demonstrated, with a virus transporter, that the subthalamic nucleus of the basal ganglia projects disynaptically to the cerebellar cortex (Bostan et al, 2010). Additionally, Rogers et al. (2011) proposed two glutamatergic pathways to connect the cerebellum and mPFC. The first network involves the lateral nucleus, reticulotegmental nuclei, pedunculopontine nuclei, ventral tegmental area (VTA), and finally mPFC (Forster and Blaha, 2003). The second arrives at the MPFC through the lateral nucleus and mediodorsal and ventrolateral thalamus (Rogers et al, 2011). Both neuronal circuities seem to contribute equally to cerebellar modulation of mPFC dopamine release. Studies in humans have observed that lesions or alterations of the cerebellum can generate similar deficits to those caused by alterations of the prefrontal cortex (Schmahmann and Sherman, 1998; Strata et al, 2011; Timmann et al, 2010). A greater release of dopamine in the cerebellar vermis has also been observed in humans after a hyperstimulation of the prefrontal cortex (Yoon et al, 2006). In addition, several anatomical studies in animals have found a dopaminergic direct VTA-cerebellar projection (Ikai et al, 1992, 1994) and non-dopaminergic direct projections from the DCN to the VTA (Carta et al, 2019; Ikai et al, 1992; Watabe-Uchida et $a l, 2012$ ). Some of these studies observed a connection between the prefrontal cortex and cerebellum through dopaminergic projections to the cerebellar cortex and nondopaminergic to the DCN (Ikai et al, 1992). Ikai et al. (1994) showed that VTA dopaminergic fibres reach both the granular layer and the Purkinje layer. On the other hand, the non-dopaminergic fibres from the VTA reached the DCN, mainly the lateral and interpositus nuclei. These non-dopaminergic projections were also observed in the opposite direction, from the DCN to the VTA, with a contralateral predominance (Carta et al, 2019; Ikai et al, 1994). Additionally, the medial and interpositus nuclei send projections to the thalamus (Stanton, 1980) and the lateral nucleus project to the caudate and putamen (Hoshi et al, 2005). 


\section{The cerebellum in addiction}

The role of the cerebellum has traditionally been related to posture, motor control, and coordination. However, as it has been demonstrated in the last decades, the cerebellum also participates in brain functions such as emotional memory and behaviour (Adamaszek et al, 2017; Sacchetti et al, 2002a, 2004; Strata et al, 2011; Turner et al, 2007), linguistic processing (Mariën et al, 2014), planning, prediction and temporal perception (Courchesne and Allen, 1997), reward (Wagner et al, 2017), and decisionmaking (Blackwood et al, 2004; Broche-Pérez et al, 2016; Moers-Hornikx et al, 2009).

As above mentioned, several anatomical studies have shown that the cerebellum connects anatomically and functionally with the addiction circuitry (Bostan et al, 2018; Buckner et al, 2011; Carta et al, 2019; Chen et al, 2014; Hoshi et al, 2005; Ichinohe et al, 2000; Ikai et al, 1992; Middleton and Strick, 2000, 2001; Panagopoulos et al, 1991; Sang et al, 2012; Stanton, 1980; Watabe-Uchida et al, 2012; Xiao et al, 2018). Moreover, it is known that drug use promotes neuroplasticity processes and reorganization in the prefronto-cerebellar circuits (Miquel et al, 2009, 2016; Moulton et al, 2014).

Volumetric studies of magnetic resonance imaging (MRI) have observed smaller cerebellums in drug addicts (Barros-Loscertales et al, 2011; Gallinat et al, 2006; Lin et al, 2012; Shear et al, 1996; Sim et al, 2007). A study conducted in methamphetamineaddicted individuals showed an increase in grey matter volume in multiple cortical regions (angular and temporal gyrus, precuneus, insula, and occipital pole), but a reduction in the cerebellum as compared with the normal sample, even after one month of abstinence (Morales et al, 2012).

Additionally, functional magnetic resonance imaging (fMRI) studies have repeatedly demonstrated activations in the cerebellum of addicted individuals during cue-reactivity tasks for all drugs of abuse. In a group of cocaine abusers exposed to drug-related cues, metabolic increases were described in the dorsolateral prefrontal cortex, amygdala, and cerebellum (Grant et al, 1996). Another study in cocaine addicts showed left hemispheric activation of lateral amygdala, lateral orbitofrontal cortex, and rhinal cortex, as well as right hemispheric activation of the dorsolateral prefrontal cortex and cerebellum after presentation of drug-related cues (Bonson et al, 2002). Cocaine- 
associated cues elicit selectively activation in lobules II, III, VIII, and IX of the vermis (Anderson et al, 2006). Increases in baseline fMRI activity have also been observed bilaterally in the ventral striatum and cerebellum in heroin addicts (Li et al, 2015). In alcoholic patients, craving-elicited odour cues activate the right amygdala, hippocampus, insula, and cerebellum (Schneider et al, 2001). Remarkably, opioiddependent patients present decreased functional connectivity at rest between reward structures (NAC and amygdala) and the cerebellum, including Crus I (Upadhyay et al, 2010).

It has been assumed that a main role of the basal ganglia in reward prediction and reward-based learning, while the cerebellum would be involved in adaptive modification of behaviour and error-based learning (Doya, 2000). However, fMRI research showed that reward prediction error in a Pavlovian reward association task correlated not only with striatum but also with cerebellar activity (O'Doherty et al, 2003). More importantly, granule cell activity appeared to encode the expectation of reward (Wagner et al, 2017). In this way, the cerebellum may play an influential modulatory role in reward/saliency, as it also shares reciprocal connections with dopaminergic systems in the basal ganglia (Bostan and Strick, 2010). Strongly supporting the cerebellum's role in reward, Carta et al. (2019) have very recently shown that an optogenetic stimulation of the cerebellar axons in the VTA was as rewarding as direct optogenetic stimulation of dopaminergic neurons within the VTA.

Recent research from our lab found a specific and distinctive cerebellar hallmark of preference for cues linked to chronic cocaine experience. We have shown that cocaineinduced conditioned preference significantly increases the activity in neurons located in the most dorsal part of the cerebellar cortex in the vermis (the apical part) (Carbo-Gas et al, 2014a, 2014b). This pattern was not observed when animals did not develop conditioned preference, despite being treated with the same cocaine dose (Carbo-Gas et al, 2014a, 2014b). More specifically, the cerebellar activity correlated with preference only in lobule VIII. Thus, lobe VIII could be especially relevant for addiction since it is one of the components of the sensorimotor (Bostan et al, 2013; Schmahmann, 1991) and the limbic networks (Adamaszek et al, 2017), working as an interface among sensory processing, emotional states, and motor responses. The posterior vermis has 
been proposed as "the limbic cerebellum" (Bostan et al, 2013; Strata et al, 2011; Timmann et al, 2010; Turner et al, 2007). Therefore, lobe VIII would exhibit an advantageous position when making predictions using drug-conditioned memories to evoke preparatory operations in motor networks that may automatically activate drugseeking (Carbo-Gas et al, 2014b). Supporting this hypothesis, the posterior vermis has been involved in the automation of behaviour repertoires towards drug-related cues (Yalachkov et al, 2010).

In conclusion, all these findings indicate that different cerebellar regions participate in executive control, drug-induced memory, response selection, and salience (Goldstein and Volkow, 2002, 2011; Habas et al, 2009; Volkow et al, 2010) further highlighting the cerebellum's potential role in addiction.

\section{Drug-induced plasticity changes in the cerebellum}

It has been observed that the acute and chronic experience with cocaine affects both the activity and plasticity in the cerebellum. Chronic cocaine use is related to the brain interactions between glutamate and dopamine, which have also been found in the cerebellum (Schweighofer et al, 2004). More concretely, it has been observed that levels of the NR2C subunits of the glutamate receptor were reduced in the rat cerebellum during the late withdrawal from cocaine (Yamaguchi et al, 2002). In addition, extracellular activity records in the cerebellar cortex showed that cocaine administration can decrease the spontaneous activation of Purkinje cells and glutamate-evoked discharges (Jiménez-Rivera et al, 2000). Other studies observed an increase of cFos levels in the granular layer of the cerebellar vermis (Carbo-Gas et al, 2014a, 2014b), mediated via D1 receptors in rats treated chronically with d-amphetamine or cocaine (Klitenick et al, 1995).

The changes that cocaine causes in molecular and structural plasticity of the cerebellum appear already at the short term, but they seem to require an incubation time (VazquezSanroman et al, 2015a, 2015b). In mice, the direction of cerebellar plasticity changes depends on the duration of the withdrawal period that precedes a new cocaine experience (Vazquez-Sanroman et al, 2015a, 2015b). After a one-week withdrawal, a new cocaine administration promoted an increase in proBDNF levels and its expression 
in Purkinje neurons, while no changes were observed in the expression of mature BDNF (Vazquez-Sanroman et al, 2015a). Moreover, cocaine-treated mice showed an increase in D3 receptor levels and internal expression of Glu2 subunit of the AMPA receptor. Interestingly, these changes were associated with pruning of dendritic spines and a reduction in the size and density of the Purkinje synaptic terminals. The cocaine-induced effects impaired the inhibitory Purkinje function over the DCN associated with a decrease in the probability of remodelling in the Purkinje-DCN synapses, due to an upregulation of perineuronal nets (PNNs) that surround the medial nuclear neurons (Vazquez-Sanroman et al, 2015a). Conversely, one-month withdrawal period induced an increase in both proBDNF and mature BDNF levels in the cerebellum (VazquezSanroman et al, 2015b). Externalization of GluR2 expression was selectively increased in the soma and dendrites of Purkinje cells in the posterior cerebellum. Additionally, we found more dendritic branching and larger axon terminals in Purkinje neurons to be associated with the increased balanced expression of BDNF. This kind of plasticity accompanied a reduction in PNN expression in the DCN that might facilitate the subsequent remodelling of Purkinje-DCN synapses.

PNNs are a specialised extracellular matrix, composed of chondroitin sulphate proteoglycans surrounding the soma and proximal dendrites of several neuronal populations, which expression restricts neuronal plasticity to stabilize circuits (Brückner et al, 1993; Carulli et al, 2006; Foscarin et al, 2011). Consequently, PNN expression has been proposed as a stabilization mechanism for plasticity changes in learning and memory (Gogolla et al, 2009; Romberg et al, 2013; Tsien, 2013).

In addition, PNNs have been involved in the long-lasting expression of drug plasticity changes. Similar effects of PNN disruption through the use of chrondroitinase $A B C$ have been observed in different brain regions, and a large number of studies support a PNN's role in drug-induced memories (Blacktop et al, 2017; Van den Oever et al, 2010; Slaker et al, 2015, 2016; Xue et al, 2014). PNN degradation in the prelimbic, but not in the infralimbic cortex, reduced acquisition and reconsolidation of cocaine-induced conditioned place preference (CPP) (Slaker et al, 2015), whereas the digestion of PNNs in the amygdala prevented priming-induced drug reinstatement as long as degradation was made before extinction (Xue et al, 2014). Furthermore, it has been shown that 
deletion of PNNs in the anterior dorsal lateral hypothalamic area abolished the acquisition of cocaine-induced CPP and significantly diminished self-administration of cocaine (Blacktop et al, 2017). In a recent study in our laboratory, animals exposed to cocaine-induced preference conditioning showed that the formation of cocaine-related preference memories increased the expression of PNNs surrounding Golgi inhibitory interneurons and the activity of these Golgi cells surrounded by strong and fully condense PNNs in the dorsal region of lobule VIII. However, the expression of PNNs in the medial nucleus was reduced in all cocaine-treated groups and thereby was not memory related (Carbo-Gas et al, 2017). All these results suggest that the regulation of PNNs around Golgi neurons in the cerebellar cortex might be a relevant mechanism for the stabilization of drug-related memories.

Overall, findings using drugs of abuse support the previously demonstrated role of the cerebellum in the formation of Pavlovian memories (Boele et al, 2010; Sacchetti et al, 2002b, 2005; Thompson and Steinmetz, 2009), suggesting that the posterior vermis is an important region for the persistence of drug-related memories. More importantly, as we previously proposed (Miquel et al, 2009), the relevance of the cerebellum in addiction may increase as far as the activity of the prefrontal cortex is reduced. In the present doctoral thesis, we proposed for the first time a hypothetical model to explain prefrontal-cerebellar interactions for the establishment of cocaine-related conditioned memory. 
AIMS AND HYPOTHESIS 



\section{AIMS AND HYPOTHESIS}

The premises underpinning the present thesis are the following:

- The cerebellum is a fundamental region for the consolidation of Pavlovian and instrumental conditioned memories.

- The cerebellum has connections with the prefronto-striatal circuits previously related to addictive behaviour.

- Addictive drugs, and specifically cocaine, promote stable neuroplastic changes in the cerebellum.

- Activity in the posterior cerebellum increases in mice that developed preference for cocaine-related cues.

The general aim of the thesis is to investigate the role of prefronto-cerebellar networks in cocaine-induced conditioned memory. More specifically, the present thesis is aimed at: (1) studying the function of the prefronto-cerebellar circuits in the acquisition of cocaine-induced conditioned preferences; (2) investigating the effects of cerebellarprefrontal manipulations on neural activity and plasticity; and (3) proposing a working hypothetical model to explain cerebellum-prefrontal relationships in drug addiction.

Specific aims:

- To explore the effects produced by deactivations of the medial prefrontal cortex, comparing prelimbic and infralimbic cortices on the acquisition of cocaineinduced preference conditioning.

- To explore the effects produced by deactivations of the posterior cerebellum, specifically lobe VIII of the vermis on the acquisition of cocaine-induced preference conditioning.

- To evaluate the effects of simultaneous prefrontal-cerebellar deactivations on cocaine-induced preference conditioning.

- To investigate whether mPFC deactivations could promote plasticity changes in the cerebellum by analysing neuronal activity (cFos) and one of the mechanisms for synaptic stabilization, the expression of PNNs, associated with the acquisition of cocaine-induced conditioned memory. 
- To investigate whether impairment in the posterior cerebellar cortex may affect activity and PNN expression in the MPFC and the striatum.

The present thesis proposes, as a general hypothesis, that the posterior cerebellum plays a fundamental role in the storage of cocaine-related memories. In this way, our predictions were:

- Infralimbic deactivation will facilitate the acquisition of cocaine-induced conditioned memory, while prelimbic impairment will prevent its acquisition.

- Animals with deactivations of lobe VIII in the vermis will not acquire cocainerelated memory.

- No effect of the deactivations will be observed in animals trained under a random cocaine-cue association.

- Activity and plasticity in the cerebellum will be promoted after mPFC deactivations.

- Deactivation of lobule VIII will change the activity and plasticity in the MPFC and the striatum. 
EXPERIMENTAL STUDIES

Chapter 1 



\title{
The role of the cerebellum in drug-cue associative memory: functional interactions with the medial prefrontal cortex
}

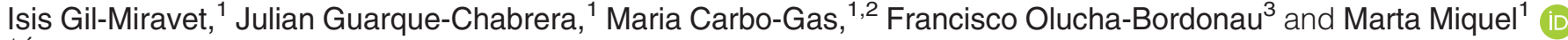 \\ ${ }^{1}$ Área de Psicobiología, Universitat Jaume I, Castellón de la Plana, Spain \\ ${ }^{2}$ INSERM U1215, Pathophysiology of Addiction, NeuroCentre Magendie, Bordeaux, France \\ ${ }^{3}$ UP Medicina, Universitat Jaume I, Castellón de la Plana, Spain
}

Keywords: cocaine, drug-induced conditioning, lidocaine, odours, quinolinic acid

\begin{abstract}
Drug-induced Pavlovian memories are thought to be crucial for drug addiction because they guide behaviour towards environments with drug availability. Drug-related memory depends on persistent changes in dopamine-glutamate interactions in the medial prefrontal cortex (mPFC), basolateral amygdala, nucleus accumbens core and hippocampus. Recent evidence from our laboratory indicated that the cerebellum is also a relevant node for drug-cue associations. In the present study, we tested the role that specific regions of the cerebellum and MPFC play in the acquisition of cocaine-induced preference conditioning. Quinolinic acid was used to manage a permanent deactivation of lobule VIII in the vermis prior to conditioning. Additionally, lidocaine was infused into the prelimbic and infralimbic (IL) cortices for reversible deactivation before every training session. The present findings show, for the first time, that the cerebellum and mPFC might act together in order to acquire drug-cue Pavlovian associations. Either a dorsal lesion in lobule VIII or an IL deactivation encouraged cocaine-induced preference conditioning. Moreover, simultaneous IL-cerebellar deactivation prevented the effect of either of the separate deactivations. Therefore, similar to the IL cortex, neural activity in the cerebellum may be crucial for ensuring inhibitory control of the expression of cocaine-related memories.
\end{abstract}

\section{Introduction}

The strength and persistence of drug-seeking responses in drug addiction are thought to be sustained by long-lasting drug-cue associative memories that compel goal-directed behaviours towards contexts of drug availability (Everitt and Robbins 2005). The incentive and conditioned reinforcing properties of drug-related cues depend on persistent changes in dopamine-glutamate interactions in the medial prefrontal cortex (mPFC), basolateral amygdala (BLA), nucleus accumbens core (NAcore) and hippocampus (Belin and Everitt 2008; Volkow et al. 2013).

Remarkably, the cerebellum is closely connected to the functional loops in the striatum-cortico-limbic circuitry, which has been established by tracing techniques, electrostimulation, and optogenetics (Panagopoulos et al. 1991; Ikai et al. 1992; Hoover and Strick 1999; Ichinohe et al. 2000; Melchitzky and Lewis 2000; Hoshi et al. 2005; Glaser et al. 2006; Yu et al. 2007; Bostan et al. 2010; Chen et al. 2014; Herrera-Meza et al. 2014). Moreover, different regions in the cerebellum have been demonstrated to be involved in

Correspondence: Marta Miquel, as above. E-mail: miquel@uji.es

dited by John Foxe. Reviewed by Ryan LaLumiere and Guy Mittleman.

Received 28 February 2018, revised 14 June 2018, accepted 28 June 2018

All peer review communications can be found with the online version of the article. the formation and storage of motor and emotional Pavlovian memory (Steinmetz et al. 1992; Topka et al. 1993; Sacchetti et al. 2002, 2004; Gao et al. 2016; Giovannucci et al. 2017). Additionally, growing evidence has indicated that the cerebellum is a relevant node for drug-cue associations in humans (Moulton et al. 2014) and animals (Carbo-Gas et al. 2014a,b, 2017). Neuroimaging studies of cue reactivity in drug addicts have consistently shown activation in the cerebellum when drug-related cues were presented (Grant et al. 1996; Schneider et al. 2001; Bonson et al. 2002; Anderson et al. 2006; Fuentes et al. 2012). Recent research from our laboratory has gone a step further in determining an accurate location for the cerebellar area involved in these drug-cue associations (Carbo-Gas et al. 2014a,b, 2017). Overall, our findings have indicated that cocaineinduced preference conditioning selectively increases neural activity and the expression of perineuronal nets in the dorsal region of the granular cell layer in the vermis. Correlations between neural activity and drug-induced conditioned preference were observed in lobules III, VIII and IX. These cerebellar lobules receive dopaminergic projections from the ventral tegmental area (VTA) (Ikai et al. 1992, 1994) and express dopamine transporters (Melchitzky and Lewis 2000; Carbo-Gas et al. 2014b).

Several studies have observed that the prelimbic (PL) and infralimbic (IL) cortices form different reciprocal loops through the brain (Ongür and Price 2000; Vertes 2004; Hoover and Vertes 2007) and 
exhibit opposite roles at the functional level (Ongür and Price 2000; McFarland and Kalivas 2001; Capriles et al. 2003; Peters et al. 2009; Sierra-Mercado et al. 2011; Ball and Slane 2012; Pfarr et al. 2015). Specifically, reinstatement of cocaine-seeking behaviour requires the integrity of the PL cortex (McFarland and Kalivas 2001; McLaughlin and See 2003), whereas the IL cortex is needed for the suppression of this response, presumably promoting the extinction of this behaviour (LaLumiere et al. 2010; Lalumiere et al. 2012).

Thus, two different reciprocal loops have been proposed for the mPFC. Reinstatement of cue-induced cocaine seeking is driven by close interactions among the PL, NAcore, BLA and VTA. By contrast, the consolidation and expression of extinction of a previously acquired cocaine seeking response are under the control of the IL, NAshell and BLA (McFarland and Kalivas 2001; McLaughlin and See 2003; Lalumiere et al. 2012).

Interestingly, human studies of drug addicts have indicated that the prefrontal cortex and cerebellum may be recruited in a competitive manner during reward tasks (Martin-Sölch et al. 2001; Desmond et al. 2003; Hester 2004; Bolla et al. 2005). In these studies, a prefrontal impairment was accompanied by strong activation of the cerebellum. Thus, it seems that the cerebellum acquires higher functional relevance when prefrontal function is compromised by disease or chronic drug use (Anderson et al. 2006; Miquel et al. 2009).

Very recently, we proposed that the dorsal and ventral regions in the posterior vermis could be functionally related to different prefrontal-striatal-limbic loops in order to initiate or restrain cocaine seeking (Miquel et al. 2016). In the present investigation, we tested for the first time the role that specific regions of the cerebellar cortex play in the acquisition of cocaine-induced conditioned preference. Additionally, we explored the effects of focal deactivation in the IL and PL cortices. Finally, we wondered whether simultaneous IL-cerebellum deactivation would be able to change the effects of deactivating each of the regions separately. Importantly, this work is the first attempt to provide support for a causative role of the cerebellum in the regulation of drug-related behaviours.

\section{Methods}

\section{Subjects}

Male Sprague-Dawley rats weighing $175-200 \mathrm{~g}(N=151)$ were obtained from Janvier (ST Berthevin Cedex, France). Rats were individually housed in the animal facility (Jaume I University, Spain) under standard laboratory conditions (12-h light cycle from 8:00 AM to 8:00 PM) with access to food and water ad libitum. Handling was performed on a daily basis for 2 weeks before the experiments began. Rats were subjected to stereotaxic surgery when they reached a weight of $270-350 \mathrm{~g}$. Behavioural protocols took place within the first 5 hours of the light cycle, 2 hours after the lights were turned on. All animal procedures were approved by the local Animal Welfare Ethics Committee and Empowered Body and were developed in accordance with the European Community Council directive (2010/63/EU), Spanish directive BOE 34/11370/2013 and local directive DOGV 26/2010.

\section{Pharmacological agents}

Cocaine hydrochloride (Alcaliber S.A., Madrid, Spain) was dissolved in a $0.9 \%$ saline solution and administered intraperitoneally (IP). The $0.9 \%$ saline solution was used as the control vehicle. Anaesthesia was induced using a cocktail of ketamine $(100 \mathrm{mg} / \mathrm{kg})$ (Imalgene $100 \mathrm{mg} / \mathrm{mL}$; Mersal Laboratorios S.A., Barcelona,
España) and xylazine $(10 \mathrm{mg} / \mathrm{kg}$ ) (xylazine hydrochloride $\geq 99 \%$ : Sigma-Aldrich Co. LLC, Madrid, España). Lidocaine $(6 \% ; 60 \mathrm{mg} /$ $\mathrm{mL}$ ) (lidocaine hydrochloride: Sigma-Aldrich Co. LLC) and quinolinic acid $(90 \mathrm{nmol} / \mu \mathrm{l})$ (2,3-pyridinedicarboxylic acid: SigmaAldrich Co. LLc) were used for deactivation of the mPFC and the cerebellum respectively.

\section{Stereotaxic surgery and brain deactivation procedures}

All rats weighed between 270 and $350 \mathrm{~g}$ before stereotaxic surgery. Surgery was performed using a Kopf stereotaxic apparatus. For the intracranial infusion, a stainless steel guide cannula (length, $10 \mathrm{~mm}$; external diameter, 23 gauge) was targeted at the following coordinates with respect to bregma (Paxinos and Watson 1998). For the cerebellum, the dorsal area (AP: - 14.5; ML: 0; DV: -4.5) and the ventral area (AP: -13 ; ML: 0; DV: -4.5$)$ of lobule VIII in the vermis were targeted. For the mPFC, the PL (AP: +3.2; ML: +0.6/-0.6; DV: -3 ) and IL (AP: +3.2; ML: + 0.6/-0.6; DV: -4$)$ cortices were targeted (Fig. 1). After the surgery, all the animals received analgesic treatment with meloxicam (Metacam $5 \mathrm{mg} / \mathrm{mL}$; Boehringer Ingelheim España S.A., Barcelona, España), repeated every 24 hours for 3 days. The animals remained undisturbed for 3 to 5 days after surgery for recovery (for the experimental timeline see Fig. 2A).

Excitotoxic lesions from quinolinic acid were preferred for the lesion of the posterior cerebellum (lobule VIII) because in our past experience, the cannula installation did not remain in place for a long time. In this case, the infusion was performed only once during the initial surgery under anaesthesia. Quinolinic acid $(90 \mathrm{nmol} / \mu \mathrm{l})$ was released through a removable stainless steel injector (length, $11 \mathrm{~mm}$; external diameter, 30 gauge) inserted into the previously implanted guide cannula and connected to an infusion pump (volume, $0.5 \mu \mathrm{l}$; infusion ratio, $0.2 \mu \mathrm{l} / \mathrm{min}$ ). The infusions were made unilaterally at the middle line of lobule VIII in the vermis (ML: 0), which is in this cerebellar region that we have previously described plasticity changes linked to cocaine-related memory (Carbo-Gas et al. 2014a,b, 2017). After the infusion was completed, the injector remained in place for 3 minutes to avoid liquid aspiration. Then, the guide cannula was removed, and the wound was sutured. The same procedure was implemented in the sham group, but in this case, phosphate buffered saline (PBS) was infused.

For mPFC deactivations, the guide cannulas were attached to the skull through stainless steel screws fixed with acrylic dental cement. Stainless steel obturators were kept in the guide cannula to maintain the cannula's integrity. Rats were gently handled while restrained, and $6 \%$ lidocaine $(60 \mathrm{mg} / \mathrm{mL})$ was infused either into the IL or PL cortex before each training trial (volume, $1 \mu \mathrm{l}$; infusion ratio, $0.5 \mu \mathrm{l} / \mathrm{min}$ ). Rats were not anaesthetised during the microinjections because this procedure does not involve pain or discomfort for the animals. Behavioural trials began 2 minutes after the infusion, as deactivation via lidocaine only lasts for 20 minutes (Martin 1991). Sham animals underwent the same procedure, but saline was infused instead of lidocaine. Cannula placements for each site were counterbalanced among the animals in terms of the right and left sides, and infusions were made unilaterally. Bilateral cannula installations were not included in this study as we intended to preserve mPFC functions partially in order to obtain a more realistic picture of what would happen during an early chronic experience with the drug or in vulnerable brains.

Finally, simultaneous deactivations of the cerebellum and IL cortex were achieved using the two abovementioned procedures in the same rat. Therefore, rats were trained under a unilateral IL deactivation together with a neurotoxic lesion in the dorsal region of lobule VIII. The rationale behind this study was to test whether these two 


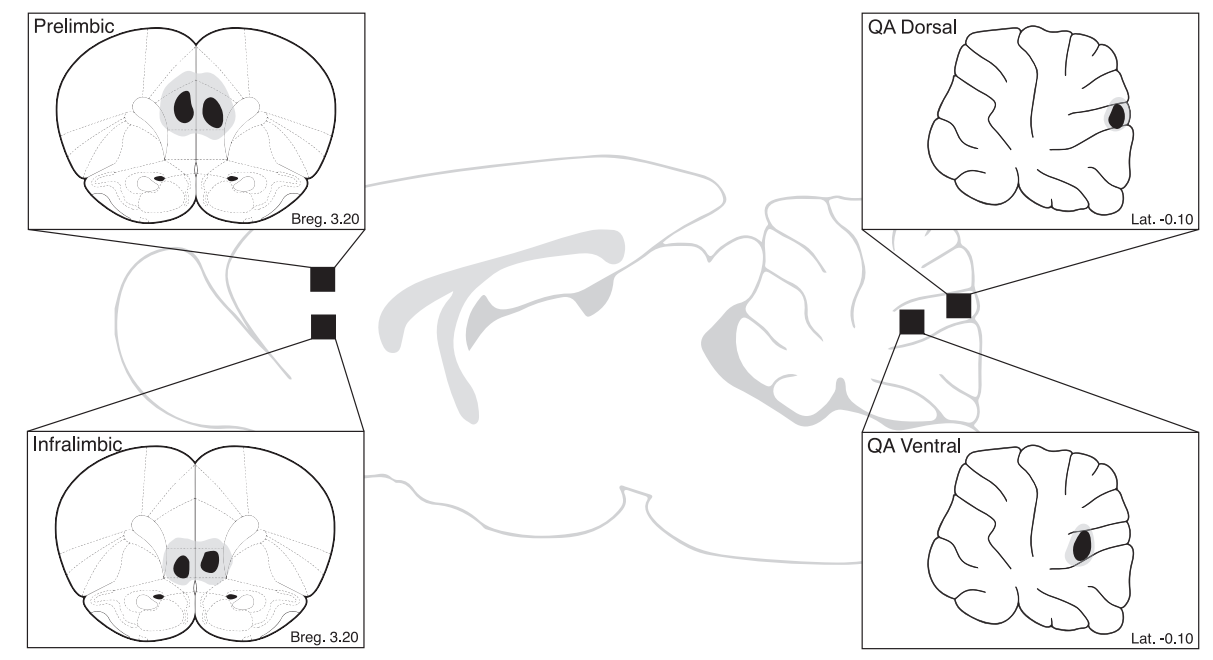

FIG. 1. Diagrams of the cannula locations. Schematic diagrams depicting the largest (grey) and smallest (black) diffusion areas in the PL and IL cortices, as well as in the dorsal and ventral regions of the cerebellar vermis. The extent of the diffusion areas was assessed using light microscopy and lucida camera drawings.

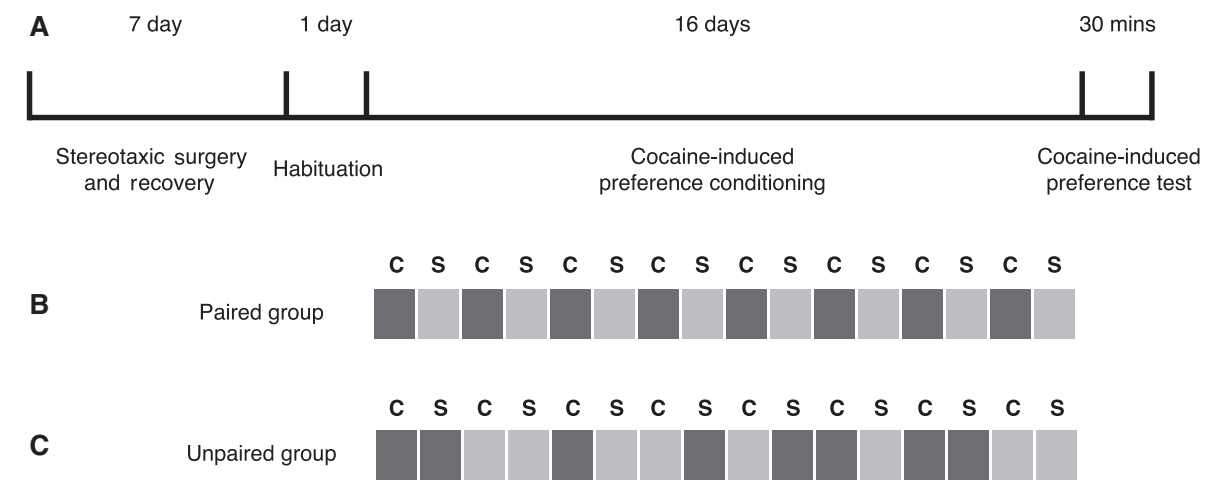

FIG. 2. (A) Experimental timeline. Different stages of the experimental procedure from the stereotaxic surgery to the animal perfusion. (B) Cocaine-induced preference conditioning protocol for the paired group. For 16 training days, rats received eight cocaine ' $\mathrm{C}$ ' and eight saline ' $\mathrm{S}$ ' administrations on alternate days that were associated with olfactory stimuli that acted as the CS+ (dark grey) or CS- (light grey). (C) Cocaine-induced preference conditioning protocol for the unpaired group. The number of cocaine ' $\mathrm{C}$ ' and saline ' $\mathrm{S}$ ' injections was the same as previously mentioned, but they were randomly associated with the odours.

regions might act to compensate each other after impairment of any of them. In this case, one could expect that the effect of separate deactivations would be prevented.

\section{Cocaine-induced preference conditioning procedure}

Conditioning was developed in an opaque, oblong corridor $(90 \times 20 \times 60 \mathrm{~cm})$ that included two lateral black chambers $(20 \times 20 \times 60 \mathrm{~cm})$ located on opposite sides. We evaluated the initial preference for two olfactory stimuli (lavender and rose) of four animals. Because the innate preferences for the odours were not different [Student's $t$-test for dependent samples: $t(3)=0.8692, P=0.4487$ ], these two equally preferred odours were used in the conditioning experiment. Two drops of lavender or rose fragrance were put on gauze and presented inside a steel ball with holes that hung on the walls of the chambers. One of the odours acted as the conditioned stimulus (CS+) and was associated with cocaine $(15 \mathrm{mg} / \mathrm{kg}$, IP). On alternate days, rats were exposed to the other odour (CS) and received saline injections. During the training session, the animals remained confined in one of the lateral chambers, and access to the other side was blocked by a panel. Each pairing session lasted for $15 \mathrm{~min}$. A total of eight cocaine-cue paired sessions were conducted, and the odours used as the CS+ and CS-, as well as the left and right locations in the corridor, were counterbalanced among the animals (Fig. 2B).
Preference for the cocaine-related cue was evaluated 48 hours after the last cocaine administration in a 30 minutes drug-free test in which the CS+ and CS - were presented simultaneously on both sides of the corridor. Importantly, the location of the odours (CS+ and $\mathrm{CS}-$ ) was opposite to that in the training. Therefore, for the first 10 minutes of the test session, the animals were allowed to explore the new location of the cues, and thus, this period was not included in the analysis. Then, the time spent (TS) in each chamber was recorded for the last $20 \mathrm{~min}$. All the test sessions were videotaped and scored by a blind observer. The preference score was calculated as [TS in (CS+)/TS in $(\mathrm{CS}+)+\mathrm{TS}$ in $(\mathrm{CS}-)] \times 100$. Additionally, we included a pseudoconditioning group (Unp group) that was treated with the same number of cocaine injections but was randomly associated with both olfactory stimuli (Fig. 2C). These unpaired groups allowed us to test for memory-related effects of our brain deactivations.

\section{Locomotor activity}

Activity was scored by a blind observer in the videos obtained from the preference test session. The 20-minute testing period was split into four segments of $5 \mathrm{~min}$. The number of crossovers was registered by dividing the corridor into four equal quadrants on the screen. A locomotion score was assigned each time an animal crossed over from one quadrant to another on all four legs. Locomotion was assessed only 
during the preference test. During conditioning, motor activity was not considered, as rats were confined to one of the lateral chambers for the entire session. Thus, despite the fact that free movement was possible inside these boxes, the movement was limited to a very short distance.

\section{Perfusion protocol and brain sampling}

Animals were deeply anaesthetised with sodium pentobarbital $(30 \mathrm{mg} / \mathrm{kg}$ ) (Dolethal $100 \mathrm{~mL}$; Vetoquinol E.V.S.A., Madrid, España) 90 minutes after the preference test and were perfused transcardially, first with saline solution $(0.9 \%)$ and then with paraformaldehyde (4\%). After perfusion, the brain and cerebellum were quickly dissected and placed in a container with the same fixative for 24 hours at $4{ }^{\circ} \mathrm{C}$. After this time, the tissue was immersed in a $30 \%$ sucrose solution in PBS until the brain sank. The brain tissue was rapidly frozen by quick immersion in liquid nitrogen, and $40-\mu \mathrm{m}$ sections were performed with a cryostat microtome (Microm HM560; Thermo Fisher Scientific, Barcelona, Spain). Four series of tissue sections were collected and stored at $-80{ }^{\circ} \mathrm{C}$ in cryoprotectant solution with ethylene glycol. Sagittal sections of the cerebellum and brainstem were selected according to the lateral coordinates from -0.72 to $0.72 \mathrm{~mm}$, comprising the whole vermis. For the prefrontal cortex, coronal sections were collected according to bregma coordinates from 4.70 to $1.70 \mathrm{~mm}$ (Paxinos and Watson 1998). Several sections were stained with cresyl violet for assessment of the cannula locations. Lesion sites were identified and represented using light microscopy and camera lucida drawings. Rats with cannula misplacement were used as negative controls and were not included in the statistical analysis (Fig 1).

\section{Experimental design and statistics}

All behavioural data were based on the preference scores obtained on the test day. Statistical analyses were performed using GraphPad Prism 7 software (GraphPad Software Inc., La Jolla, CA, USA). As a first step, we analysed the effect of cerebellar and prefrontal deactivations on cocaine-induced preference conditioning. In this analysis, because the normality requirements were met, the results were presented as the mean \pm SEM and were analysed by one-way ANOVA or Student's $t$-tests for independent samples. Then, post hoc comparisons were performed using Tukey's HSD tests. As a second step, we used an arbitrary cut-off point of $60 \%$ to cluster sham rats in two subgroups: the preference $(\geq 60 \%$, Sham P) and no preference $(<60 \%$, Sham NP) groups. The rationale behind the use of a cut-off point to conform these two different subgroups was based on our previous findings that indicated a completely different kind of cocaine-induced plasticity when comparing mice expressing preference with those that did not (Carbo-Gas et al. 2014a,b, 2017). Comparisons of the variances in these groups were carried out using Kruskal-Wallis nonparametric analyses tests with post hoc Dunn's multiple comparison test. The results were depicted by scatter plots and median scores. For the data regarding the proportion of rats expressing preference scores higher than $60 \%$, a chi-square test was used to determine differences between the expected vs. observed frequencies. In all analyses, the statistical level of significance was set at $P<0.05$.

\section{Results}

The injection sites are shown in Fig 1. As can be seen, focal infusions with very small diffusion areas were achieved in the present study. Neither of the sham deactivations produced significant effects on cocaine-induced preference conditioning, as demonstrated by Student's $t$-tests for independent samples [Sham Dorsal vs. Sham Ventral: $(t(19)=0.6104, P=0.5489)$; Sham IL vs. Sham PL: $(t$ $(17)=0.7523, P=0.4622)]$. Therefore, sham animals were collapsed for each brain region to shape two different control groups, namely, the Sham cerebellum and Sham mPFC groups. Then, these two groups were split into preference $(\mathrm{P})$ and no preference (NP) groups, as explained above. Additionally, we tested for significant differences between the effects of deactivation on the left and right sides of the mPFC. Neither the sham $[(t(17)=1.05, P=0.3085)]$ nor the lidocaine groups [IL $(t(6)=1.299, P=0.2418)$; PL $(t$ $(5)=0.06548, P=0.9503)]$ exhibited any kind of lateralisation effect.

\section{An excitotoxic lesion in the dorsal region of lobule VIII facilitates cocaine-induced preference conditioning}

A one-way ANOVA of the preference scores yielded a significant group effect $(F(2,32)=4.672, P=0.0166)$. As shown by subsequent post hoc comparisons using Tukey's HSD tests, the quinolinic acid dorsal group (QA Dors) $(n=6)$ exhibited a significantly higher preference for the CS+ than the control (Sham) $(n=21)$ $(P=0.0143)$ and unpaired dorsal (Unp Dors) $(n=8)(P=0.0492)$ groups (Fig. 3A).

As seen in figure $3 \mathrm{~A}$, only a subgroup of the sham rats showed a clear preference for the cocaine-related odour cue. Therefore, the sham animals were split into two subgroups, namely, the Sham NP $(n=15)$ and Sham $\mathrm{P}(n=6)$ groups, by using the arbitrary preference cut-off point of $60 \%$. A Kruskal-Wallis test demonstrated a significant effect of the group factor $(\mathrm{H}$ $(4)=23.06, P<0.0001)$. Post hoc comparisons revealed that all lesioned animals (QA Dors) showed the same preference level as that of the Sham $\mathrm{P}$ group $(P>0.99)$ (Fig. 3B), and both groups exhibited an increased preference for the CS+ compared to that of the Sham NP group $(P<0.001)$. Then, a chi-square test was conducted to compare the proportion of animals that met our criteria for preference in each group. Remarkably, the excitotoxic lesions in the dorsal lobule VIII promoted the acquisition/expression of cocaine-induced preference conditioning in $100 \%$ of the trained animals $\left(\chi^{2}(2)=10.89, P=0.0043\right)$. However, the percentage of preference animals in the sham group was $28.57 \%$ (Fig. 3C).

\section{Rats with a ventral region of lobule VIII do not show cocaine- induced preference conditioning}

A one-way ANOVA comparing the preference for the CS+ did not demonstrate a significant effect of the group factor $(F(2,36)=1.301$, $P=0.2848)$ (Fig. 3D). Nevertheless, the nonparametric analysis, which split the sham group into the NP $(n=15)$ and $\mathrm{P}(n=6)$ groups, yielded a significant effect of the group factor $(H(4)=16.31$, $P<0.001$ ) (Fig. 3E). Dunn's multiple comparisons test revealed that ventrally lesioned animals (QA Vent) showed a similar preference score to those of the Sham NP $(P>0.99)$ and unpaired ventral (Unp ventral) groups $(P>0.99)$. In addition, the Sham $P$ group exhibited a higher preference than the Sham NP $(P<0.001)$ and QA Ventral $(P<0.004)$ groups (Fig. 3E). Despite the fact that no lesioned animal reached the preference score of $60 \%$, a chi-square test revealed no significant differences in the proportions of rats that acquired cocaineinduced conditioned preference after ventral lesions $\left(\chi^{2}(2)=3.954\right.$, $P=0.138$ ) (Fig. 3F). 
A
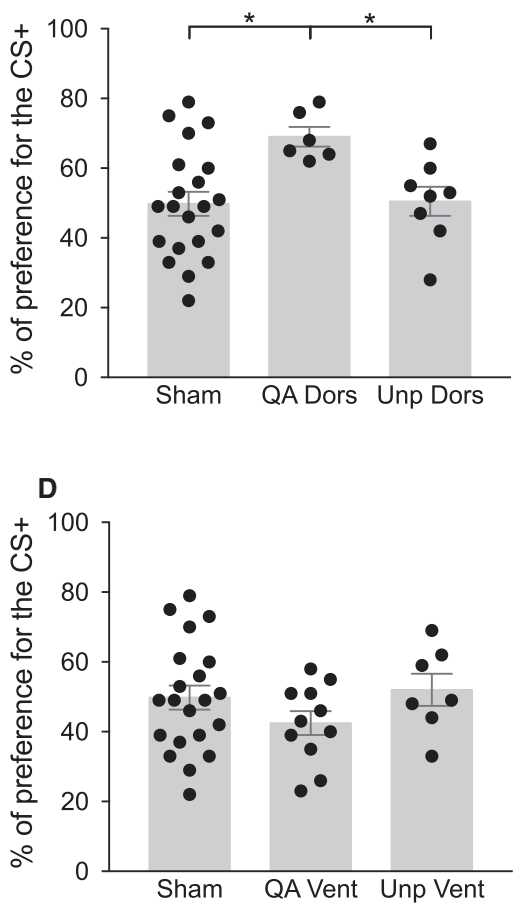

B
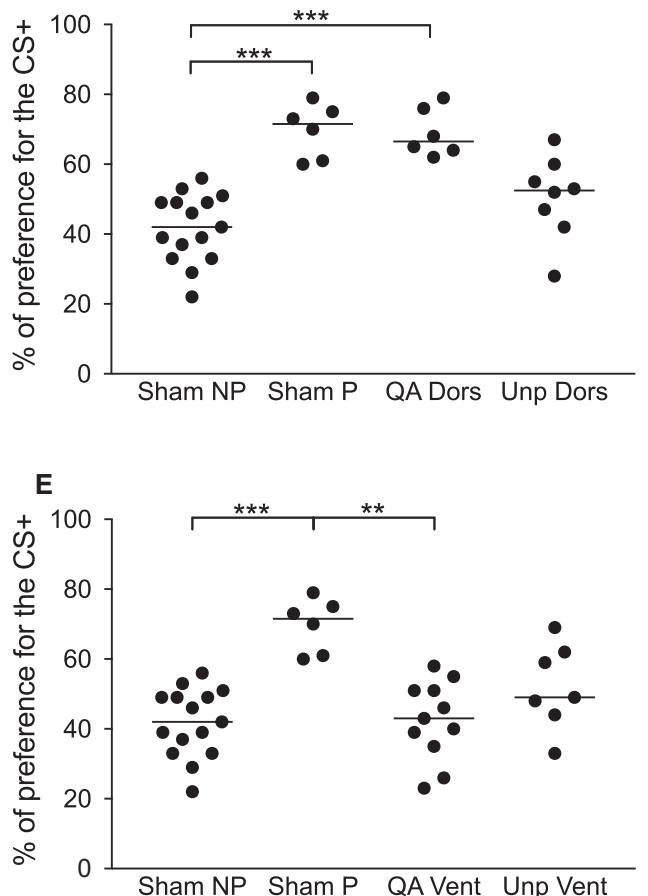

C
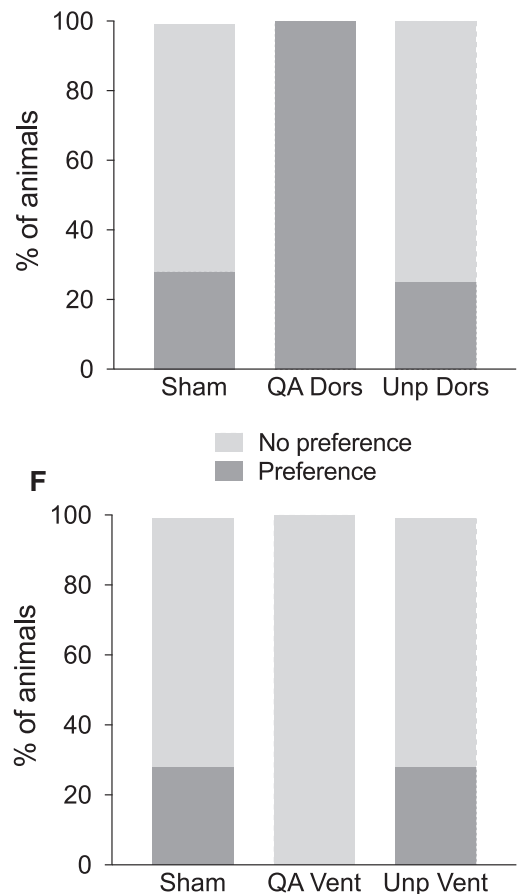

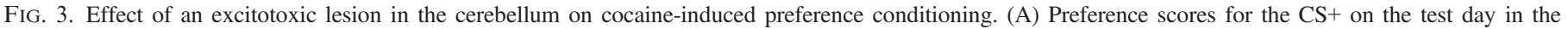

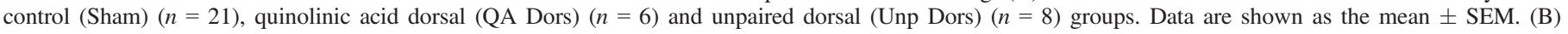

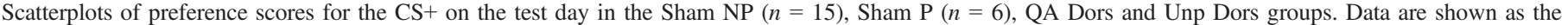

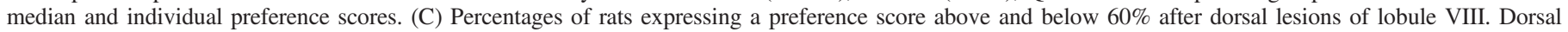

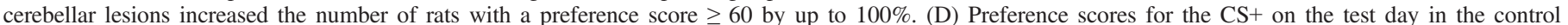

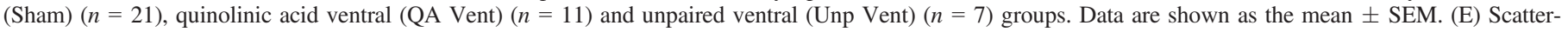

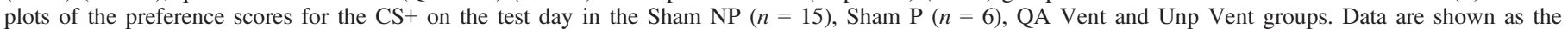

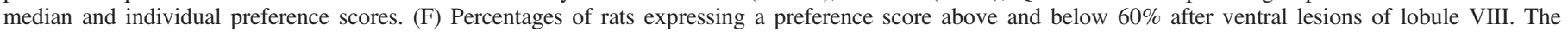
lesions prevented rats from expressing a preference towards the cocaine-related cue. $(* P<0.05 ; * * P<0.01 ; * * * P<0.001)$.

\section{A temporary deactivation of the IL cortex promotes cocaine- induced preference conditioning}

A temporal deactivation of the IL cortex facilitated the acquisition of cocaine-induced preference conditioning, as indicated by a oneway ANOVA $(F(2,31)=8.879, P=0.0009)$. As shown by a subsequent post hoc comparison using Tukey's HSD tests, the lidocaine IL group (Lido IL) $(n=8)$ exhibited a significantly higher preference for the CS+ than the sham $(n=19)(P=0.0021)$ and unpaired lidocaine groups (Unp IL) $(n=7)(P=0.0021)$ (Fig. 4A). The Kruskal-Wallis test showed a significant effect of the group factor (H (4) $=21.3, P<0.0001$ ) (Fig. 4B). Dunn's post hoc comparisons revealed that a repeated IL deactivation before each training session increased preference to the same level as that shown by the Sham $\mathrm{P}$ group $(P>0.99)$. Additionally, both groups were different from the Sham NP group $(P<0.001$ and $P<0.03$ respectively), but only the animals in the lidocaine IL group exhibited a significantly higher preference than the unpaired group $(P<0.02)$ (Fig. 4B). Moreover, $100 \%$ of deactivated animals expressed a preference score higher than $60 \%\left(\chi^{2}(2)=10.6, P=0.005\right)($ Fig. $4 \mathrm{C})$.

\section{Rats with a temporary deactivation of the PL cortex do not show cocaine-induced preference conditioning}

The transient deactivation of the PL cortex did not produce a significant effect on cocaine-induced conditioned preference ( $F$ $(2,31)=1.152, P=0.3293$ ) (Fig. 4C). Nevertheless, as seen in the scatter plots (Fig. 4D), PL-deactivated animals $(n=7)$ showed a preference score similar to that of the Sham NP group $(n=12)$. A Kruskal-Wallis test demonstrated a significant effect of the group factor $(\mathrm{H}(4)=13.14, P=0.0043)$. Both the Sham NP $(P<0.01)$ and lidocaine PL $(P<0.05)$ groups were different from the Sham $\mathrm{P}$ group $(n=7)$, as revealed by post hoc tests (Fig. 4D). However, a chi-square test of the proportions of animals that met the criterium for preference revealed no significant differences $\left(\chi^{2}(2)=3.579\right.$, $P=0.167$ ) (Fig. 4F).

\section{Simultaneous deactivation of the IL cortex and dorsal lobule VIII prevents the facilitative effect on cocaine-induced preference conditioning}

Remarkably, the effect of IL deactivation was very similar to that observed after dorsal lesions of the cerebellar cortex. Therefore, we managed to deactivate both regions simultaneously in order to ascertain if these regions might outweigh the lack of activity in the other region after impairment. As expected if they were functionally related, the facilitative effect of the separate deactivations was prevented by combining both a unilateral deactivation of the IL cortex and a dorsal lesion of lobule VIII. Student's $t$-test for independent samples supported no differences in the preference scores between the animals with deactivation and sham animals $(t(9)=0.8126$, $P=0.4374$ ) (Fig 5A). Thus, the proportion of rats expressing preference was rescued to control levels $\left[\left(\chi^{2}(1)=0.5051, P=0.4773\right)\right]$ (Fig. 5B). 
A

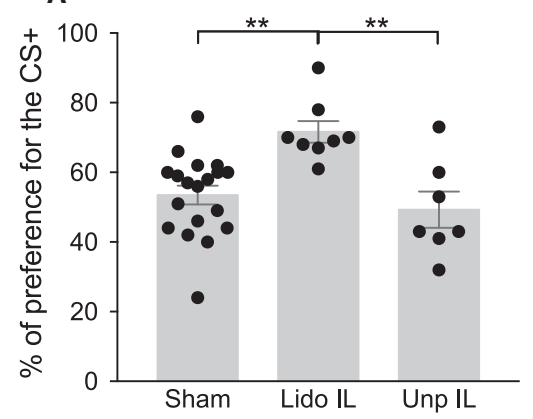

D

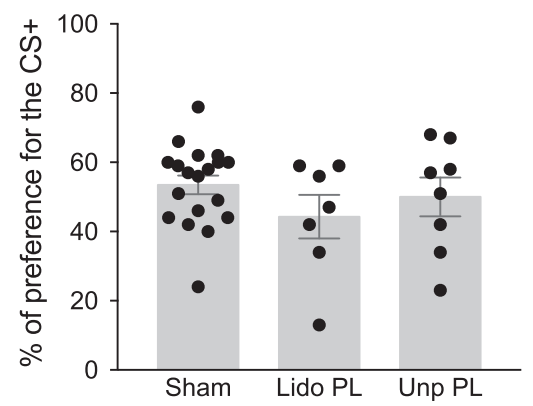

B

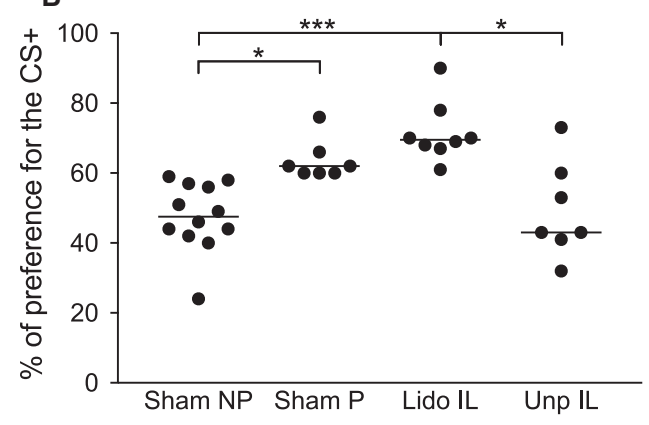

E

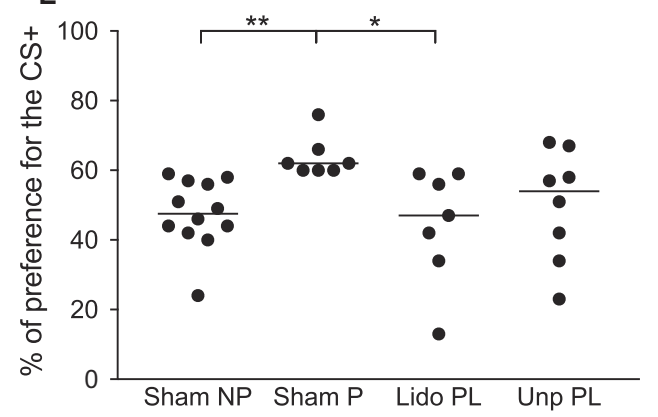

c

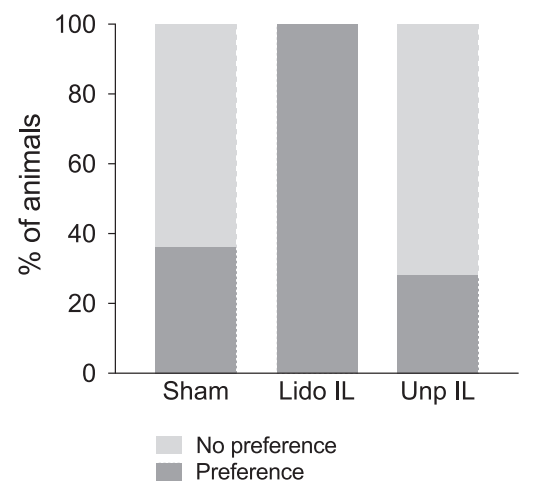

$\mathbf{F}$

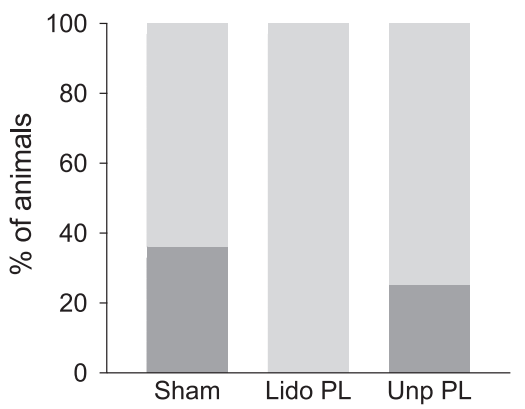

FIG. 4. Effect of a temporary deactivation in the mPFC before each training session on cocaine-induced conditioned preference. (A) Preference scores for the $\mathrm{CS}+$ on the test day in the Sham $(n=19)$, lidocaine infralimbic (Lido IL) $(n=8)$ and unpaired infralimbic (Unp IL) $(n=7)$ groups. Data are shown as the mean \pm SEM $\left(*^{* *}<<0.01\right)$. (B) Scatterplots of the preference scores for the CS+ on the test day in the Sham NP $(n=12)$, Sham P $(n=7)$, Lido IL and Unp IL groups. Data are shown as the median and individual preference scores. (C) Percentage of rats expressing a preference score above and below $60 \%$ after the deactivation of the IL cortex. The IL deactivation increased the number of rats showing a preference score $\geq 60$ by up to $100 \%$. (D) Preference scores showed by the control (Sham) $(n=19)$, lidocaine prelimbic (Lido PL) $(n=7)$ and unpaired prelimbic (Unp PL) $(n=8)$ groups on the test day. Data are shown as the mean \pm SEM. (E) Preference scores for the CS + on the test day in the Sham NP $(n=12)$, Sham P $(n=7)$, Lido PL and Unp PL groups. Data are shown as the median and individual preference scores. (F) The percentage of rats expressing a preference score above and below $60 \%$ after the deactivation of PL. The IL lesion dramatically reduced the proportion of rats that expressed cocaine-induced conditioned preference. $\left({ }^{*} P<0.05 ; * * P<0.01 ; * * * P<0.001\right)$.

\section{Motor activity during the preference test is unaffected by either prefrontal or cerebellar deactivations}

Locomotion was assessed during the preference test by dividing the 20-minute testing period into 5 minute segments. Neither of our manipulations affected locomotion during the preference test, as was demonstrated by two-way repeated measures ANOVAs of each region. In all cases, locomotion decayed during the session for all groups independent of the group factor [Dorsal cerebellum: Group $F$ $(2,10)=0.888, P=0.4415$; Time $F(3,15)=35.72, \quad P<0.0001$; Interaction $F(6,30)=2.338 ; P=0.0569]$; [Ventral cerebellum: Group $F(2,12)=2.16 P=0.1581$; Time $F(3,18)=25.85$, $P<0.0001$; Interaction $F(6,36)=2.212, P=0.0642$ ]; [IL: Group $F(2,12)=1.293, P=0.31$; Time $F(3,18)=33.04, P<0.0001$; Interaction $F \quad(6,36)=0.728, \quad P=0.0629] ; \quad[\mathrm{PL}$ : Group $F$ $(2,12)=0.489, P=0.6247$; Time $F(3,18)=18.43, P<0.0001$; Interaction $F(6,36)=2.282, P=0.0573$ ] (Fig. 6).

\section{Discussion}

It is widely accepted that mPFC impairment is a crucial part of the physiopathology of drug addiction (McFarland and Kalivas 2001; Van den Oever et al. 2010; Goldstein and Volkow 2011). However, not until recently has the cerebellum been considered a relevant structure in understanding the persistent drug-induced behavioural alterations in addiction (Miquel et al. 2009, 2016; Moulton et al. 2014).

The present results show, for the first time, that the dorsal region of the posterior cerebellum plays a role similar to that of the IL cortex in the establishment of drug-cue Pavlovian memory. The loss of activity in either of these regions dramatically increased the number of animals that expressed cocaine-induced conditioned preference. The effects of a lesion in the ventral region of lobule VIII or a deactivation of the PL cortex are less clear. In both cases, the inactivation seems to reduce the proportion of rats that show preference for the cocaine-related cue, although statistics do not provide full support for the significance of the effects. Thus, further research is needed in order to propose any functional interactions between these two regions. Importantly, as all the effects were memory-related and specific for the formation of drug-cue associations, none of our manipulations were shown to be effective in the pseudo-conditioned rats (unpaired groups).

We noticed that in our procedure only a small group of control rats $(29 \%)$ developed a clear preference for the cocaine-related cue. Several methodological issues, such as the high cocaine dose used $(15 \mathrm{mg} / \mathrm{kg})$, the use of a discrete odour cue instead of a place preference procedure or the elevated number of drug-cue pairings, might explain the reduced number of conditioned animals found in the sham groups. Nevertheless, both IL and dorsal cerebellar impairment caused a consistent and robust effect, increasing by up to $100 \%$ the number of animals expressing conditioned preference (Figs 34C-C).

As the IL deactivation was unilateral, the intact contralateral $\mathrm{IL}$ or even the PL cortex might increase its activity, promoting the acquisition of cocaine-related memory. As a matter of fact, the PL cortex may be inhibited by the IL cortex (McFarland and Kalivas 2001; Lalumiere et al. 2012), and thus, the facilitative effect on cocaine-induced 
A

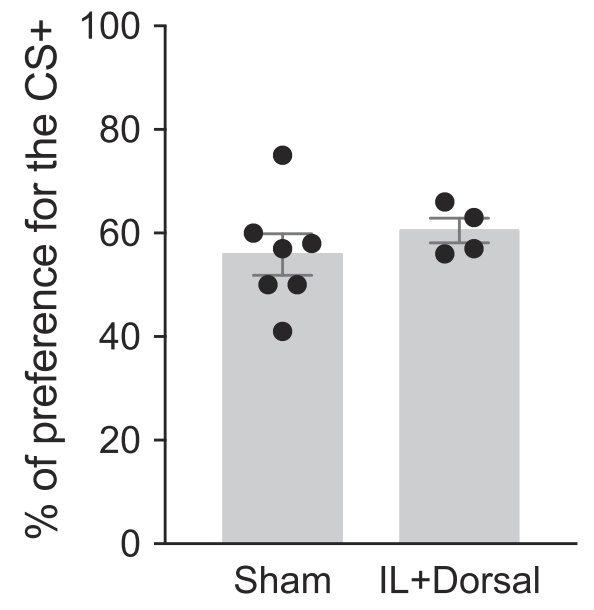

B

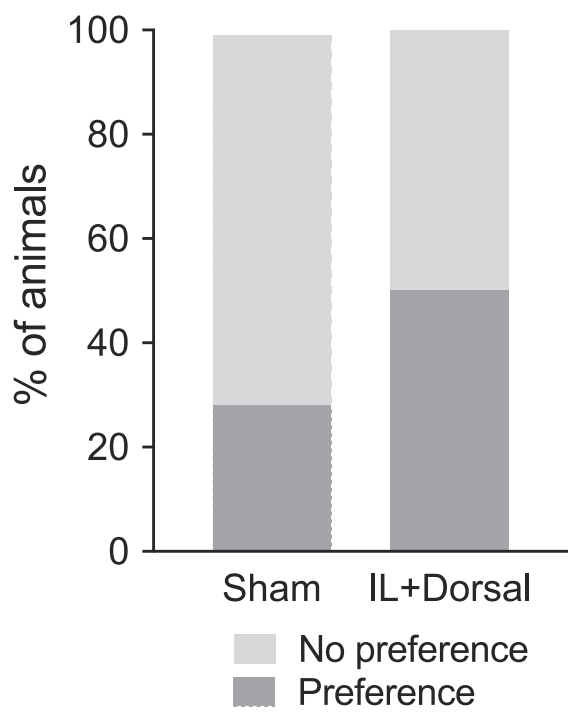

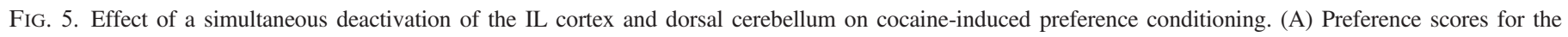

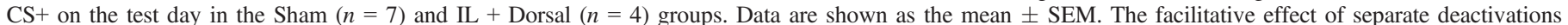

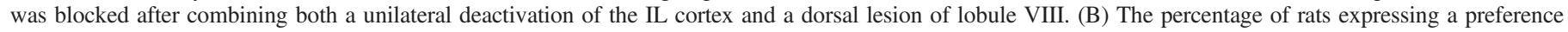

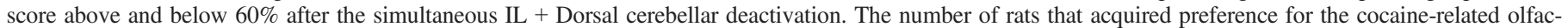
tory stimulus was similar to that in the control group.
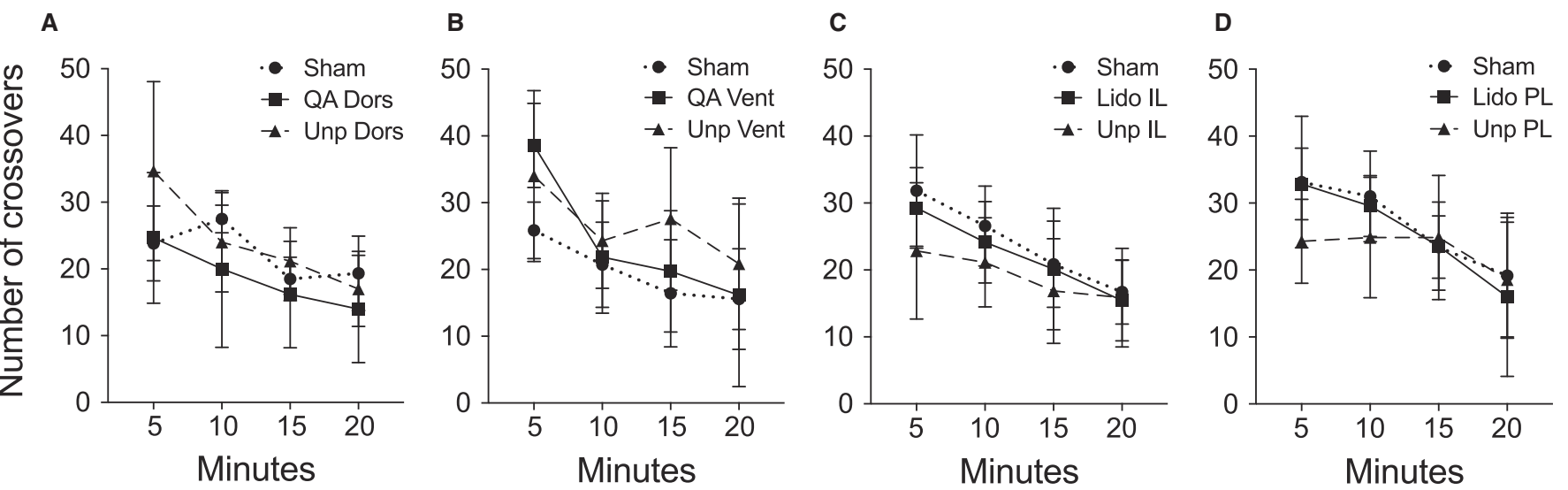

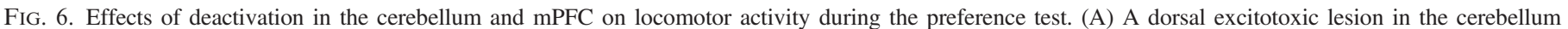

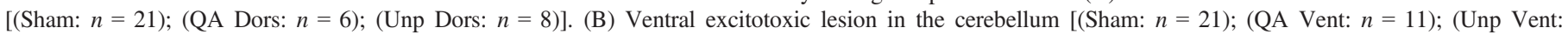

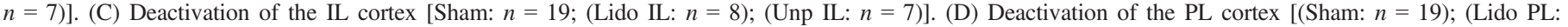

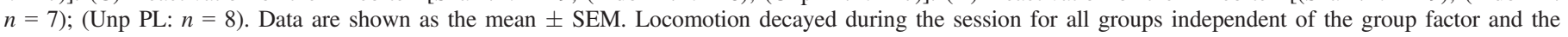
region deactivated.

conditioning, caused by a partial IL deactivation, might then be explained by a reduced inhibition of PL activity. IL deactivation could also enhance activity in the cerebellum. Indeed, it has been shown that the prefrontal dysfunction observed in drug addicts is accompanied by an increase in cerebellar activity (Martin-Sölch et al. 2001; Desmond et al. 2003; Hester 2004; Bolla et al. 2005). Similarly, lesions of the dorsal cerebellum could boost neural activity in the mPFC, striatum and limbic regions and thereby facilitate cocaine-induced preference conditioning. Supporting a close functional loop, our results also revealed that simultaneous cerebellum-IL impairment prevents the facilitative effect of separate deactivations. This finding suggested that both the IL cortex and the dorsal cerebellum might increase their relevance during conditioning when the other region has been compromised. Subsequently, if both areas are impaired at the same time, this compensatory function will not be possible, and the propensity to acquire cocaine-induced conditioned preference will resemble that of the control group.

The present findings argued in favour of our recent hypothesis proposing that the dorsal regions of the posterior vermis are part of the IL-NAshell-BLA network (Miquel et al. 2016). Our previous work established that the plasticity hallmark signatures of cocaineinduced preference conditioning are expressed in the dorsal region of the cerebellar cortex (Carbo-Gas et al. 2014a,b). Strikingly, we showed here that the acquisition of drug-cue associations is facilitated when the same region of the cerebellar cortex is damaged. Taken together, our findings suggested that the dorsal region of the posterior vermis might inhibit drug seeking using previously learned Pavlovian associations that involve other additional regions in the striatum-cortico-limbic circuitry. Interestingly, behavioural inhibition has been one of the functions ascribed to the cerebellum 
(Moers-Hornikx et al. 2009; Picazio and Koch 2015). Cerebellar lesions promote behavioural disinhibition (Schmahmann and Sherman 1998; Tanaka et al. 2003), whereas increasing activity in the cerebellum improves inhibitory control (Brunamonti et al. 2014).

Numerous studies have found reciprocal loops between the prefrontal cortex and the cerebellum that may provide anatomical evidence to explain the present results (Middleton and Strick 1994, 2001; Schmahmann and Pandya 1997; Sang et al. 2012; see Bostan and Strick 2018 for a recent compelling review). Moers-Hornikx et al. (2009) observed an increase in cFos expression in the deep cerebellar nuclei and the prefrontal cortex after deep brain stimulation of the mediodorsal and ventrolateral thalamic nuclei in rats. Furthermore, cortical regulation of striatal activity can be modulated by the cerebellum (Chen et al. 2014). A direct dopaminergic VTAcerebellar projection has also been demonstrated (Ikai et al. 1992, 1994). Detectable DA levels were found in the posterior lobules of the vermis (VII-X), the right and left hemispheres and the fastigial, interpositus and dentate nuclei (Glaser et al. 2006). In addition, it has been shown that the cerebellar cortex may regulate dopamine release in the $\mathrm{mPFC}$ by several independent pathways. First, the cerebellum connects to the VTA through the reticulotegmental and pedunculopontine nuclei (Forster and Blaha 2003). Second, the cerebellum projects to the VTA through the mediodorsal and ventrolateral thalamus (Rogers et al. 2011). Finally, and more relevant for the present discussion is the finding of a direct projection from the deep cerebellar nuclei to the VTA (Watabe-Uchida et al. 2012). This projection would be crucial for explaining the present results as it provides a direct pathway for the cerebellum to control the cortico-striatal circuitry through an increase in dopaminergic activity.

Nevertheless, a number of caveats and limitations of the present study should be considered. Our sample only included rats with focal lesions in lobule VIII. It is noteworthy that other anterior or posterior cannula locations (lobule VII or IX) did not seem to reproduce the facilitative effect on cocaine-induced preference conditioning. Recent evidence indicated that the cerebellum is subdivided into different specialised regions to regulate specific behaviours (Glickstein et al. 2011; Witter and De Zeeuw 2015). However, it has also become clear that a cerebellar lobule is not the main functional unit. First, a lobule can contain several functional areas; second, cerebellar functions can encompass several lobules (Witter and De Zeeuw 2015). This raises the question of which functional characteristics and connectivity patterns make the dorsal region of lobule VIII in the vermis somehow relevant to associative memory and behavioural inhibition. The dorsal cerebellar cortex receives sensorimotor corticopontine and exteroceptive components of the mossy fibre afferent system, providing neural information from cortical sensorimotor networks to the cerebellum (Ekerot and Larson 1972; Voogd and Ruigrok 2004). In addition, a recent study of motor associative learning established a prominent nucleocortical excitatory projection of mossy fibres to the most superficial region of the granule cell layer that optimised the conditioned response (Gao et al. 2016). Granule cell activity in this area is present during unconditioned and conditioned stimuli, as well as during the conditioned response (Giovannucci et al. 2017). This activity also encodes the expectation of reward (Wagner et al. 2017). Classically, lobule VIII in the vermis was considered part of the skeletomotor divisions of the cerebellum, projecting to motor cortices through the fastigial nucleus and also to the descending motor pathways (Glickstein et al. 2011). It is important to highlight that the present cerebellar lesion did not cause a generalised and unspecific motor disinhibition because locomotor activity during the preference test was not affected. Lobules VII-X of the vermis have also been proposed to serve as an interface among sensory processing, emotional states and motor responses, due to the anatomical and functional connectivity with the amygdala and other areas of the emotional brain (Adamaszek et al. 2017). Therefore, it is plausible for the cerebellum to modulate the reward response in other areas of the striatum-cortico-limbic circuitry.

In conclusion, our findings open new avenues to understanding the role of the cerebellum in drug addiction. Further research using specific experimental approaches is needed to determine the control of different neuronal populations in the dorsal and ventral regions of the vermis.

\section{Acknowledgements}

This research was supported by the following grants and fellowships: Ministerio de Educación Cultura y Deporte (FPU12/04059); Universitat Jaume I (PREDOC2014/11); UJI (14I307.01/1); Ministerio de Economía y Competitividad (MINECO) (PSI2015-68600-P); and Plan Nacional de Drogas 2017 (PND-132400).

\section{Conflict of interest}

All authors declare no conflicts of interest.

\section{Data accessibility}

Data are available from the corresponding author upon request.

\section{Author Contributions}

All authors made a substantial contribution to the manuscript, and they were involved in critically revising the present version. Isis Gil-Miravet performed the stereotaxic surgeries and behavioural experiments; Isis Gil-Miravet, Julian Guarque-Chabrera, María Carbo-Gas and Francisco Olucha-Bordonau were involved in data analysis and the editing of the manuscript. Finally, Marta Miquel designed the study, supervised the surgeries and behavioural experiments, and drafted the manuscript. All authors approved the present version of the manuscript.

\section{References}

Adamaszek, M., D’Agata, F., Ferrucci, R., Habas, C., Keulen, S., Kirkby, K. C., Leggio, M., Mariën, P. et al. (2017). Consensus paper: cerebellum and emotion. Cerebellum, 16, 552-576.

Anderson, C. M., Maas, L. C., Frederick, B., Bendor, J. T., Spencer, T. J., Livni, E., Lukas, S. E., Fischman, A. J. et al. (2006). Cerebellar vermis involvement in cocaine-related behaviors. Neuropsychopharmacology, 31, $1318-1326$

Ball, K. T., \& Slane, M. (2012). Differential involvement of prelimbic and infralimbic medial prefrontal cortex in discrete cue-induced reinstatement of 3,4-methylenedioxymethamphetamine (MDMA; ecstasy) seeking in rats. Psychopharmacology, 224, 377-385.

Belin, D., \& Everitt, B. J. (2008). Cocaine seeking habits depend upon dopamine-dependent serial connectivity linking the ventral with the dorsal striatum. Neuron, 57, 432-441.

Bolla, K. I., Eldreth, D. A., Matochik, J. A., \& Cadet, J. L. (2005). Neural substrates of faulty decision-making in abstinent marijuana users. NeuroImage, 26, 480-492.

Bonson, K. R., Grant, S. J., Contoreggi, C. S., Links, J. M., Metcalfe, J., Weyl, H. L., Kurian, V., Ernst, M. et al. (2002). Neural systems and cueinduced cocaine craving. Neuropsychopharmacology, 26, 376-386.

Bostan, A.C., \& Strick, P.L. (2018). The basal ganglia and the cerebellum: nodes in an integrated network. Nat. Rev. Neurosci., 19, 338-350.

Bostan, A. C., Dum, R. P., \& Strick, P. L. (2010). The basal ganglia communicate with the cerebellum. Proc. Natl. Acad. Sci. USA, 107, 8452-8456.

Brunamonti, E., Chiricozzi, F. R., Clausi, S., Olivito, G., Giusti, M. A., Molinari, M., Ferraina, S., \& Leggio, M. (2014). Cerebellar damage impairs executive control and monitoring of movement generation. PLoS ONE, 9, e85997. 
Capriles, N., Rodaros, D., Sorge, R. E., \& Stewart, J. (2003). A role for the prefrontal cortex in stress- and cocaine-induced reinstatement of cocaine seeking in rats. Psychopharmacology, 168, 66-74.

Carbo-Gas, M., Vazquez-Sanroman, D., Aguirre-Manzo, L., Coria-Avila, G. A., Manzo, J., Sanchis-Segura, C., \& Miquel, M. (2014a). Involving the cerebellum in cocaine-induced memory: pattern of cFos expression in mice trained to acquire conditioned preference for cocaine. Addict. Biol., 19, $61-76$.

Carbo-Gas, M., Vazquez-Sanroman, D., Gil-Miravet, I., De las HerasChanes, J., Coria-Avila, G. A., Manzo, J., Sanchis-Segura, C., \& Miquel, M. (2014b). Cerebellar hallmarks of conditioned preference for cocaine. Physiol. Behav., 132, 24-35.

Carbo-Gas, M., Moreno-Rius, J., Guarque-Chabrera, J., Vazquez-Sanroman, D., Gil-Miravet, I., Carulli, D., Hoebeek, F., De Zeeuw, C. et al. (2017). Cerebellar perineuronal nets in cocaine-induced pavlovian memory: site matters. Neuropharmacology, 125, 166-180.

Chen, C. H., Fremont, R., Arteaga-Bracho, E. E., \& Khodakhah, K. (2014). Short latency cerebellar modulation of the basal ganglia. Nat. Neurosci., 17, 1767-1775.

Desmond, J. E., Chen, S. H. A., DeRosa, E., Pryor, M. R., Pfefferbaum, A., \& Sullivan, E. V. (2003). Increased frontocerebellar activation in alcoholics during verbal working memory: an fMRI study. Neurolmage, 19, $1510-1520$.

Ekerot, C. F., \& Larson, B. (1972). Differential termination of the exteroceptive and proprioceptive components of the cuneocerebellar tract. Brain Res., 36, 420-424.

Everitt, B. J., \& Robbins, T. W. (2005). Neural systems of reinforcement for drug addiction: from actions to habits to compulsion. Nat. Neurosci., 8, $1481-1489$.

Forster, G. L., \& Blaha, C. D. (2003). Pedunculopontine tegmental stimulation evokes striatal dopamine efflux by activation of acetylcholine and glutamate receptors in the midbrain and pons of the rat. Eur. J. Neurosci., 17, 751-762.

Fuentes, P., Barrós-Loscertales, A., Bustamante, J. C., Rosell, P., Costumero, V., \& Ávila, C. (2012). Individual differences in the behavioral inhibition system are associated with orbitofrontal cortex and precuneus gray matter volume. Cogn. Affect. Behav. Neurosci., 12, 491-498.

Gao, Z., Proietti-Onori, M., Lin, Z., ten Brinke, M. M., Boele, H. J., Potters, J. W., Ruigrok, T. J. H., Hoebeek, F. E. et al. (2016). Excitatory cerebellar nucleocortical circuit provides internal amplification during associative conditioning. Neuron, 89, 645-657.

Giovannucci, A., Badura, A., Deverett, B., Najafi, F., Pereira, T. D., Gao, Z., Ozden, I., Kloth, A. D. et al. (2017). Cerebellar granule cells acquire a widespread predictive feedback signal during motor learning. Nat. Neurosci., 20, 727-734.

Glaser, P. E. A., Surgener, S. P., Grondin, R., Gash, C. R., Palmer, M., Castellanos, F. X., \& Gerhardt, G. A. (2006). Cerebellar neurotransmission in attention-deficit/hyperactivity disorder: does dopamine neurotransmission occur in the cerebellar vermis? J. Neurosci. Methods, 151, 62-67.

Glickstein, M., Sultan, F., \& Voogd, J. (2011). Functional localization in the cerebellum. Cortex, 47, 59-80.

Goldstein, R. Z., \& Volkow, N. D. (2011). Dysfunction of the prefrontal cortex in addiction: neuroimaging findings and clinical implications. Nat. Rev. Neurosci., 12, 652-669.

Grant, S., London, E. D., Newlin, D. B., Villemagne, V. L., Liu, X., Contoreggi, C., Phillips, R. L., Kimes, A. S. et al. (1996). Activation of memory circuits during cue-elicited cocaine craving. Proc. Natl. Acad. Sci. USA, 93, 12040-12045.

Herrera-Meza, G., Aguirre-Manzo, L., Coria-Avila, G. A., Lopez-Meraz, M. L., Toledo-Cárdenas, R., Manzo, J., Garcia, L. I., \& Miquel, M. (2014). Beyond the basal ganglia: CFOS expression in the cerebellum in response to acute and chronic dopaminergic alterations. Neuroscience, 267, 219-231.

Hester, R. (2004). Executive dysfunction in cocaine addiction: evidence for discordant frontal, cingulate, and cerebellar activity. J. Neurosci., 24, $11017-11022$.

Hoover, J. E., \& Strick, P. L. (1999). The organization of cerebellar and basal ganglia outputs to primary motor cortex as revealed by retrograde transneuronal transport of herpes simplex virus type 1. J. Neurosci., 19, $1446-1463$.

Hoover, W. B., \& Vertes, R. P. (2007). Anatomical analysis of afferent projections to the medial prefrontal cortex in the rat. Brain Struct. Funct., 212, 149-179.

Hoshi, E., Tremblay, L., Féger, J., Carras, P. L., \& Strick, P. L. (2005). The cerebellum communicates with the basal ganglia. Nat. Neurosci., 8, 14911493.
Ichinohe, N., Mori, F., \& Shoumura, K. (2000). A di-synaptic projection from the lateral cerebellar nucleus to the laterodorsal part of the striatum via the central lateral nucleus of the thalamus in the rat. Brain Res., 880, 191-197.

Ikai, Y., Takada, M., Shinonaga, Y., \& Mizuno, N. (1992). Dopaminergic and non-dopaminergic neurons in the ventral tegmental area of the rat project, respectively, to the cerebellar cortex and deep cerebellar nuclei. Neuroscience, 51, 719-728.

Ikai, Y., Takada, M., \& Mizuno, N. (1994). Single neurons in the ventral tegmental area that project to both the cerebral and cerebellar cortical areas by way of axon collaterals. Neuroscience, 61, 925-934.

LaLumiere, R. T., Niehoff, K. E., \& Kalivas, P. W. (2010). The infralimbic cortex regulates the consolidation of extinction after cocaine self-administration. Learn. Mem., 17, 168-175.

Lalumiere, R. T., Smith, K. C., \& Kalivas, P. W. (2012). Neural circuit competition in cocaine-seeking: roles of the infralimbic cortex and nucleus accumbens shell. Eur. J. Neurosci., 35, 614-622.

Martin, J. H. (1991). Autoradiographic estimation of the extent of reversible inactivation produced by microinjection of lidocaine and muscimol in the rat. Neurosci. Lett., 127, 160-164.

Martin-Sölch, C., Magyar, S., Künig, G., Missimer, J., Schultz, W., \& Leenders, K. (2001). Changes in brain activation associated with reward processing in smokers and nonsmokers. Exp. Brain Res., 139, 278-286.

McFarland, K., \& Kalivas, P. W. (2001). The circuitry mediating cocaineinduced reinstatement of drug-seeking behavior. J. Neurosci., 21, 86558663.

McLaughlin, J., \& See, R. E. (2003). Selective inactivation of the dorsomedial prefrontal cortex and the basolateral amygdala attenuates conditionedcued reinstatement of extinguished cocaine-seeking behavior in rats. Psychopharmacology, 168, 57-65.

Melchitzky, D. S., \& Lewis, D. A. (2000). Tyrosine hydroxylase- and dopamine transporter-immunoreactive axons in the primate cerebellum: evidence for a lobular- and laminar-specific dopamine innervation. Neuropsychopharmacology, 22, 466-472.

Middleton, F. A., \& Strick, P. L. (1994). Anatomical evidence for cerebellar and basal ganglia involvement in higher cognitive function. Science, 266, 458-461.

Middleton, F. A., \& Strick, P. L. (2001). Cerebellar projections to the prefrontal cortex of the primate. J. Neurosci., 21, 700-712.

Miquel, M., Toledo, R., Garcia, L., Coria-Avila, G., \& Manzo, J. (2009). Why should we keep the cerebellum in mind when thinking about addiction? Curr. Drug Abus. Rev., 2, 26-40.

Miquel, M., Vazquez-Sanroman, D., Carbo-Gas, M., Gil-Miravet, I., SanchisSegura, C., Carulli, D., Manzo, J., \& Coria-Avila, G. A. (2016). Have we been ignoring the elephant in the room? Seven arguments for considering the cerebellum as part of addiction circuitry. Neurosci. Biobehav. Rev., 60 , $1-11$.

Moers-Hornikx, V. M. P., Sesia, T., Basar, K., Lim, L. W., Hoogland, G., Steinbusch, H. W. M., Gavilanes, D. A. W. D., Temel, Y. et al. (2009). Cerebellar nuclei are involved in impulsive behaviour. Behav. Brain Res., 203, 256-263.

Moulton, E. A., Elman, I., Becerra, L. R., Goldstein, R. Z., \& Borsook, D. (2014). The cerebellum and addiction: insights gained from neuroimaging research. Addict. Biol., 19, 317-331.

Ongür, D., \& Price, J. L. (2000). The organization of networks within the orbital and medial prefrontal cortex of rats, monkeys and humans. Cereb. Cortex, 10, 206-219.

Panagopoulos, N. T., Papadopoulos, G. C., \& Matsokis, N. A. (1991). Dopaminergic innervation and binding in the rat cerebellum. Neurosci. Lett., 130, 208-212.

Paxinos, G., \& Watson, C. (1998). The rat brain in stereotaxic coordinates (Fourth Edition). Academic Press Inc., San Diego, CA, ISBN: 0-12547617-5.

Peters, J., Kalivas, P. W., \& Quirk, G. J. (2009). Extinction circuits for fear and addiction overlap in prefrontal cortex. Learn. Mem., 16, 279-288.

Pfarr, S., Meinhardt, M. W., Klee, M. L., Hansson, A. C., Vengeliene, V., Schonig, K., Bartsch, D., Hope, B. T. et al. (2015). Losing control: excessive alcohol seeking after selective inactivation of cue-responsive neurons in the infralimbic cortex. J. Neurosci., 35, 10750-10761.

Picazio, S., \& Koch, G. (2015). Is motor inhibition mediated by cerebellocortical interactions? Cerebellum, 14, 47-49.

Rogers, T. D., Dickson, P. E., Heck, D. H., Goldowitz, D., Mittleman, G., \& Blaha, C. D. (2011). Connecting the dots of the cerebro-cerebellar role in cognitive function: neuronal pathways for cerebellar modulation of dopamine release in the prefrontal cortex. Synapse, 65, 1204-1212. 
Sacchetti, B., Baldi, E., Lorenzini, C. A., \& Bucherelli, C. (2002). Differential contribution of some cortical sites to the formation of memory traces supporting fear conditioning. Exp. Brain Res., 146, 223-232.

Sacchetti, B., Scelfo, B., Tempia, F., \& Strata, P. (2004). Long-term synaptic changes induced in the cerebellar cortex by fear conditioning. Neuron, $\mathbf{4 2}$, 973-982.

Sang, L., Qin, W., Liu, Y., Han, W., Zhang, Y., Jiang, T., \& Yu, C. (2012). Resting-state functional connectivity of the vermal and hemispheric subregions of the cerebellum with both the cerebral cortical networks and subcortical structures. NeuroImage, 61, 1213-1225.

Schmahmann, J. D., \& Pandya, D. N. (1997). Anatomic organization of the basilar pontine projections from prefrontal cortices in rhesus monkey. $J$. Neurosci., 17, 438-458.

Schmahmann, J. D., \& Sherman, J. C. (1998). The cerebellar cognitive affective syndrome. Brain, 121, 561-579.

Schneider, F., Habel, U., Wagner, M., Franke, P., Salloum, J. B., Shah, N. J., Toni, I., Sulzbach, C. et al. (2001). Subcortical correlates of craving in recently abstinent alcoholic patients. Am. J. Psychiatry, 158, $1075-1083$.

Sierra-Mercado, D., Padilla-Coreano, N., \& Quirk, G. J. (2011). Dissociable roles of prelimbic and infralimbic cortices, ventral hippocampus, and basolateral amygdala in the expression and extinction of conditioned fear. $\mathrm{Neu}$ ropsychopharmacology, 36, 529-538.

Steinmetz, J. E., Lavond, D. G., Ivkovich, D., Logan, C. G., \& Thompson, R. F. (1992). Disruption of classical eyelid conditioning after cerebellar lesions: damage to a memory trace system or a simple performance deficit? J. Neurosci., 12, 4403-4426.
Tanaka, H., Harada, M., Arai, M., \& Hirata, K. (2003). Cognitive dysfunction in cortical cerebellar atrophy correlates with impairment of the inhibitory system. Neuropsychobiology, 47, 206-211.

Topka, H., Valls-Solé, J., Massaquoi, S. G., \& Hallett, M. (1993). Deficit in classical conditioning in patients with cerebellar degeneration. Brain, 116, 961-969.

Van den Oever, M. C., Spijker, S., Smit, A. B., \& De Vries, T. J. (2010). Prefrontal cortex plasticity mechanisms in drug seeking and relapse. $\mathrm{Neu}$ rosci. Biobehav. Rev., 35, 276-284.

Vertes, R. P. (2004). Differential projections of the infralimbic and prelimbic cortex in the rat. Synapse, 51, 32-58.

Volkow, N. D., Wang, G. J., Tomasi, D., \& Baler, R. D. (2013). Unbalanced neuronal circuits in addiction. Curr. Opin. Neurobiol., 23, 639-648.

Voogd, J., \& Ruigrok, T. J. H. (2004). The organization of the corticonuclear and olivocerebellar climbing fiber projections to the rat cerebellar vermis: the congruence of projection zones and the zebrin pattern. J. Neurocytol., 33, 5-21.

Wagner, M. J., Kim, T. H., Savall, J., Schnitzer, M. J., \& Luo, L. (2017) Cerebellar granule cells encode the expectation of reward. Nature, 544, 96-100.

Watabe-Uchida, M., Zhu, L., Ogawa, S. K., Vamanrao, A., \& Uchida, N. (2012). Whole-brain mapping of direct inputs to midbrain dopamine neurons. Neuron, $\mathbf{7 4}, 858-873$.

Witter, L., \& De Zeeuw, C. I. (2015). Regional functionality of the cerebellum. Curr. Opin. Neurobiol., 33, 150-155.

Yu, H., Sternad, D., Corcos, D. M., \& Vaillancourt, D. E. (2007). Role of hyperactive cerebellum and motor cortex in Parkinson's disease. NeuroImage, 35, 222-233. 
EXPERIMENTAL STUDIES

Chapter 2 



\section{Chapter 2: Changes in neural activity and perineuronal net expression in the cerebellum after deactivation of the medial prefrontal cortex}

Isis Gil-Miravet ${ }^{1}$, Ignasi Melchor-Eixea ${ }^{1}$, Marta Miquel ${ }^{1 *}$

1 Área de Psicobiología, Universitat Jaume I, Castellón de la Plana, Spain.

*Corresponding author. Área de Psicobiología, Universitat Jaume I, Avenida Vicente Sos Baynat sn, 12071, Castellón de la Plana, Spain.

miquel@uji.es

orcid.org/0000-0001-9670-4210 


\section{ABSTRACT}

Prelimbic (PL) and infralimbic (IL) cortices exhibit opposite roles in drug-related behaviour. The PL cortex is involved in initiating cocaine-seeking, while the IL cortex is responsible, among other functions, for the inhibitory control over drug-seeking. Importantly, several findings support the existence of reciprocal loops between the medial prefrontal cortex (mPFC) and the cerebellum. Neuroimaging studies in human addicts with prefrontal damage have shown an overactivation of the cerebellum during reward and cognitive tasks. Thus, the cerebellum may become more relevant when prefrontal function is compromised by disease or chronic drug use. In the present research, we investigated whether impairment of the MPFC during the acquisition of cocaine-induced preference conditioning may increase drug impact on neural activity and PNN expression in the cerebellum, as well as different striatal regions. Before every training session, lidocaine was infused into PL and IL for a reversible deactivation. The results showed that IL deactivation increased the probability of developing cocaineinduced preference conditioning, while PL deactivation prevented it. Moreover, the formation of cocaine-related preference memories was associated with an increase in cFos expression and PNNs intensity in the dorsal region of the posterior cerebellum. Additionally, cocaine-induced preference memory increased cFos expression in the nucleus accumbens shell. Therefore, our findings suggest that impairment of the mPFC function increases susceptibility to the acquisition of drug-induced Pavlovian memory, but do not exhibit a direct effect of MPFC deactivations on cerebellar activity and plasticity. All this data suggest that the cerebellum might be a critical region for the storage or reactivation of conditioned associations that predict drug availability.

Keywords: cocaine, mPFC, cerebellum, lidocaine, PNNs 


\section{INTRODUCTION}

The rat medial prefrontal cortex (mPFC) includes four different functional regions: the medial precentral cortex ( $\mathrm{PrCm})$, anterior cingulate cortex $(\mathrm{AC})$, prelimbic $(\mathrm{PL})$ cortex, as well as infralimbic (IL) cortex (Edward, 1992; Heidbreder and Groenewegen, 2003; Hoover and Vertes, 2007; Ongür and Price, 2000; Vertes, 2004). The different subdivisions have been linked to a wide range of brain functions dramatically affected in drug addiction, such as working memory, decision-making, executive control, instrumental learning, and emotion (Cardinal et al, 2002; Delatour and Gisquet-Verrier, 2000; Dias and Aggleton, 2000; Heidbreder and Groenewegen, 2003; Milad and Quirk, 2002; Mogensen and Holm, 1994; Morgan et al, 1993; Ragozzino et al, 1998, 1999). Particularly, several studies have observed that the PL and IL cortices exhibit opposite roles in drug-related behaviours (Ball and Slane, 2012; Capriles et al, 2003; McFarland and Kalivas, 2001; Ongür and Price, 2000; Peters et al, 2009; Pfarr et al, 2015; SierraMercado et al, 2011). The PL cortex is involved in initiating cocaine-seeking (MartínGarcía et al, 2014; McFarland and Kalivas, 2001; Zavala et al, 2003), while the IL cortex is responsible, among other functions, for the inhibitory control over drug-seeking (Lalumiere et al, 2012; LaLumiere et al, 2010; McFarland and Kalivas, 2001; Peters et al, 2008). Thus, infusions of dopaminergic antagonists or pharmacological inactivators into the PL cortex prior to the reinstatement of cocaine self-administration decreased lever pressing for the drug (McFarland et al, 2004; McFarland and Kalivas, 2001; McLaughlin and See, 2003). Oppositely, the IL deactivation before extinction promoted cocaineseeking (Lalumiere et al, 2012; Peters et al, 2008). Consistent with an inhibitory role of the IL region, its pharmacological stimulation before relapse decreased drug-seeking (Peters et al, 2008). Notwithstanding these findings, inhibitory control seems also to require $\mathrm{PL}$ function, since optogenetic stimulation of the $\mathrm{PL}$ cortex decreased compulsive cocaine-seeking in rats (Chen et al, 2013).

The PL and IL cortices form separated reciprocal loops through the striatum-limbic circuitry (Hoover and Vertes, 2007; Ongür and Price, 2000; Vertes, 2004). Indeed, the nucleus accumbens core (NACC) receives inputs primarily from the PL cortex, whereas the nucleus accumbens shell (NAcSh) receives afferences from the IL cortex (Sesack et al, 1989; Voorn et al, 2004). The activation of the glutamatergic projection from the PL 
cortex to the NACC seemed to be critical for the reinstatement of cocaine-seeking after extinction (McFarland et al, 2003, 2004). Conversely, the inactivation of the glutamatergic projection from the IL cortex to the NAcSh restored cocaine-seeking after extinction (Lalumiere et al, 2012; Peters et al, 2008). Furthermore, the manipulation of the plasticity mechanism for synaptic stabilization within the PL and IL cortices impact differently on cocaine-related behaviour. Thus, the degradation of perineuronal nets (PNNs) around GABAergic inhibitory interneurons in the $\mathrm{PL}$, but not in the IL cortex, attenuated acquisition and reconsolidation of cocaine-induced conditioned place preference (Slaker et al, 2015).

Importantly, several findings support the existence of reciprocal loops between the mPFC and the cerebellum (Bostan and Strick, 2018; Middleton and Strick, 1994, 2001; Sang et al, 2012; Schmahmann and Pandya, 1997). Electrical stimulation of the fastigial nucleus, the main output nucleus of the vermis, evokes local action potentials and regulates the activity in the PL cortex (Watson et al, 2014). Moreover, cortical regulation of striatal activity can be modulated by the cerebellum (Chen et al, 2014). MoersHornikx et al. (2009) observed an increase in cFos expression in the deep cerebellar nuclei and the prefrontal cortex after deep brain stimulation of the mediodorsal and ventrolateral thalamic nuclei in rats. Neuroimaging studies in human addicts with prefrontal damage have shown an overactivation of the cerebellum during reward tasks, especially with increased cognitive demands (Bolla et al, 2005; Desmond et al, 2003; Hester and Garavan, 2004; Martin-Sölch et al, 2001). These results indicated that the cerebellum may become more relevant when prefrontal function is compromised by disease or chronic drug use, suggesting that both regions can be recruited to functionally compensate each other (Anderson et al, 2006; Miquel et al, 2009).

In a previous study (the first chapter), we investigated the functional relationships between the PL and IL subdivisions of the MPFC and the cerebellum in a model of cocaine-induced conditioned preference (Gil-Miravet et al, 2018). Our findings showed that a deactivation of either the apical region of lobule VIII in the vermis or the IL cortex encouraged the acquisition of cocaine-induced preference conditioning. Simultaneous deactivation of both regions prevented this facilitative effect on cocaine-related memory. However, opposite results were found after a deactivation of the PL cortex or 
deeper regions of lobule VIII. In both cases, the acquisition of preference for the cocainerelated cue was prevented.

In the present research, we investigated whether impairment of the MPFC during the acquisition of cocaine-induced preference conditioning may increase drug impact on neural activity and PNN expression in the cerebellum as well as different striatal regions.

\section{METHODOLOGY}

\section{Subjects}

The present study included 27 male Sprague-Dawley rats (Janvier, ST Berthevin Cedex, France) randomly selected from the total number of rats included in the first study with mPFC deactivation ( $\mathrm{N}=49$ ) (Gil-Miravet et al, 2018) (Chapter 1). Rats, weighing between 175 and $200 \mathrm{~g}$, were individually housed under standard laboratory conditions with temperature and humidity controlled (12 h light cycle from 8:00 a.m. to 8:00 p.m.). Access to food and water was ad libitum (Jaume I University, Spain). Animals were handled on a daily base one week before the experiment began and were habituated to all of the experimental procedures. Animal procedures were approved by the local Animal Welfare Ethics Committee and Empowered Body (2014/VSC/PEA/00208) and adhered to the European Community Council directive (2010/63/EU), Spanish directive BOE 34/11370/2013, and local directive DOGV 26/2010.

\section{Pharmacological agents}

Cocaine hydrochloride (Alcaliber S.A., Madrid, Spain) was dissolved in a $0.9 \%$ saline solution and administered intraperitoneally (IP). Saline solution was used as a control vehicle. Anaesthesia was induced using a cocktail of ketamine $(100 \mathrm{mg} / \mathrm{kg})$ (Imalgene $100 \mathrm{mg} / \mathrm{ml}$, Mersal Laboratorios S.A., Barcelona, Spain), and xylazine (10 mg/kg) (Xylazine hydrochloride $\geq 99 \%$, Sigma-Aldrich, Madrid, Spain). Lidocaine $6 \%$ (60 mg/ml) (Lidocaine hydrochloride, Sigma-Aldrich, Madrid, Spain) was used for a transient deactivation of the PL or IL. 


\section{Stereotactic surgery and temporal deactivation}

Animals weighing between 270 and $350 \mathrm{~g}$ were anaesthetised with a cocktail of ketamine/xylazine (100/10 mg/kg) (IP). Surgery was performed using a Kopf stereotaxic apparatus. For the intracranial infusion, a stainless steel guide cannula (10 mm length; 23-gauge external diameter) was placed in the PL (AP: +3.2; ML: + 0.6/-0.6; DV: -3) or IL (AP: +3.2; ML: + 0.6/-0.6; DV: -4) cortices (Paxinos and Watson, 1998) (Fig. 1). The guide cannula was applied to the skull with screws and acrylic dental cement. After surgery, all animals received analgesic treatment with meloxicam (Metacam $5 \mathrm{mg} / \mathrm{ml}$, Boehringer Ingelheim, Barcelona, Spain). Administration was repeated every 24 hours

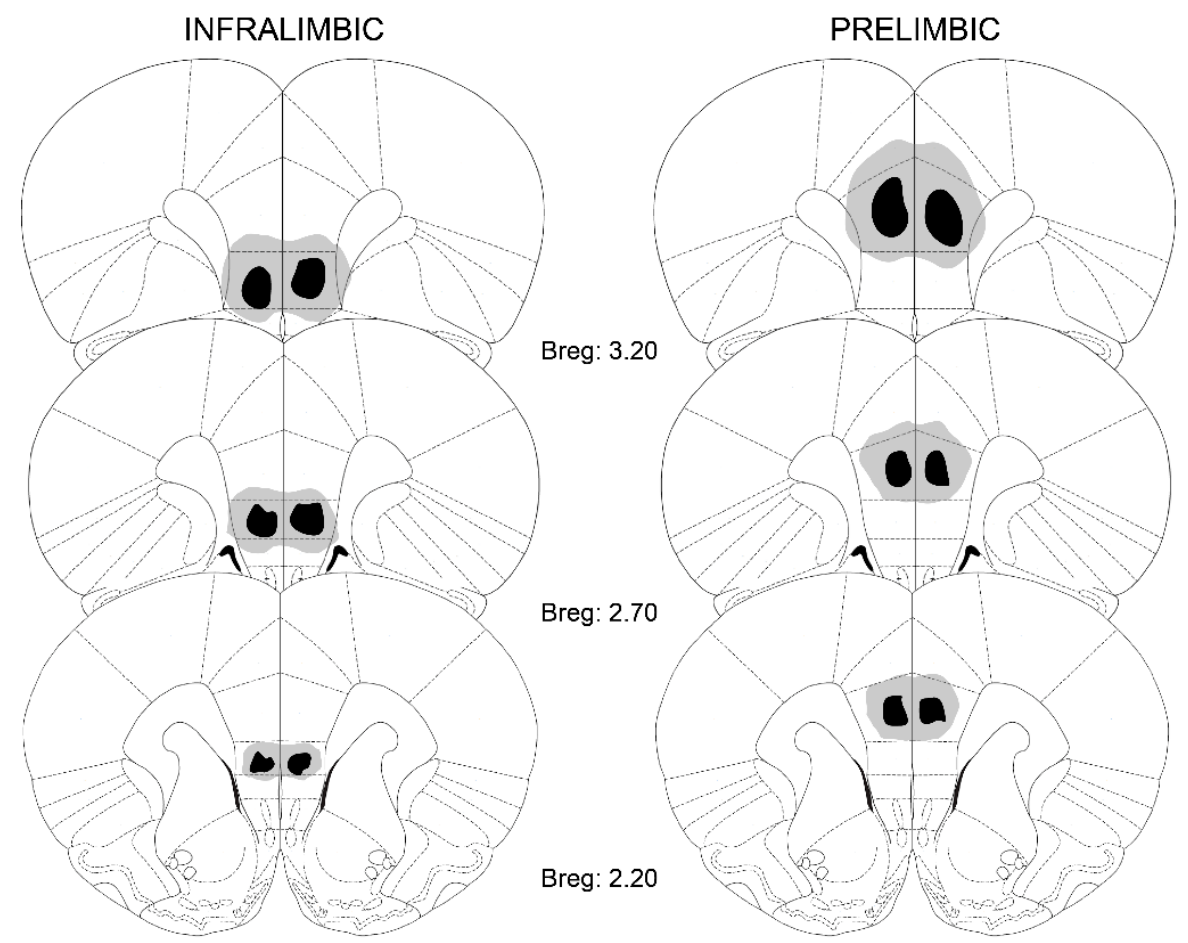

Figure 1. Diagrams of the cannula locations. Schematic diagrams depicting the largest (grey) and smallest (black) diffusion areas in the PL and IL cortices. The extent of the diffusion areas was assessed using light microscopy and camera lucida drawings.

for three days. The rats were left undisturbed for five days after surgery for recovery.

Before each training session, lidocaine $6 \%(60 \mathrm{mg} / \mathrm{ml})$ was infused in the PL or IL using an infusion pump ( $1 \mu \mathrm{l}$ volume; infusion ratio of $0.5 \mu \mathrm{l} / \mathrm{min}$ ). Microinjections were conducted in awake animals because this procedure is painless. Behavioural testing started two minutes after the lidocaine infusion. Sham surgery followed the same protocol, but saline was infused instead of lidocaine. This procedure was repeated before every conditioning trial (16 days). Cannula installations were unilateral in all the 
experiments, counterbalancing the right and left hemispheres. The rationale was to partially preserve the functions of the mPFC in order to model an early chronic experience with the drug in susceptible brains.

\section{Cocaine-induced preference conditioning procedure}

Conditioning was developed in an opaque corridor $(90 \times 20 \times 60 \mathrm{~cm})$ containing two black chambers $(20 \times 20 \times 60 \mathrm{~cm})$ located on the opposite arms (Gil-Miravet et al, 2018). We evaluated the initial preference for two odours (lavender and rose) in four animals. A Student $t$-test for independent samples $[t(6)=0.2843 ; p=0.7856$ ] revealed no preference towards any of these olfactory stimuli. Then, these two equally preferred odours were used in the conditioning experiment as previously described (Gil-Miravet et al, 2018). One of the odours was associated with cocaine (15 mg/ $\mathrm{kg}, I P)(C S+)$ and the other one with saline (CS-). The odours were counterbalanced between animals. For each pairing session, the rats were confined in one of the chambers for $15 \mathrm{~min}$. Eight cocaine- and eight saline-paired sessions were conducted on alternate days. Odour locations were also counterbalanced in the corridor.

Preference for the CS+ was evaluated $48 \mathrm{~h}$ after the last cocaine administration in a 30 min drug-free test in which CS+ and CS- odours were present simultaneously but in opposite arms in the corridor. All the test sessions were videotaped and scored by a blind observer. The first ten minutes were not considered in order to allow the animal to explore the location of the odours. Preference score was calculated as [Time Spent in CS+ / (Time Spent in CS ++ Time Spent in CS-)] $\times 100$. An additional group of rats underwent the same procedure as the deactivated animals, but cocaine injections were randomly associated with the odours (Gil-Miravet et al, 2018) (the Unp group). This pseudo-conditioned group allowed us to test for memory-related effects of prefrontal deactivations.

\section{Perfusion protocol and brain sampling}

Animals were perfused 90 min following the preference test. Animals were deeply anaesthetised with sodium pentobarbital (30 mg/kg) (Dolethal $100 \mathrm{ml}$, Vetoquinol E.V.S.A., Madrid, Spain) and perfused transcardially with saline (0.9\%) and paraformaldehyde (4\%). After perfusion, the brain and cerebellum were quickly 
dissected and placed in a container with the same fixative for $24 \mathrm{~h}$ at $4 \stackrel{\circ}{ } \mathrm{C}$. Then, the tissue was immersed in sucrose solution (30\%). The tissue was rapidly frozen by quick immersion in liquid nitrogen. Sagittal (cerebellum) and coronal (brain) sections were performed at $40 \mu \mathrm{m}$ with a cryostat microtome (Microm HM560, Thermo Fisher Scientific, Barcelona, Spain). Four series of tissue sections were collected and stored at $-80^{\circ} \mathrm{C}$ in cryoprotectant solution. Lesion sites were localized and represented using light microscopy and camera lucida drawings. Animals with cannula misplacement were not included in the statistical analysis.

\section{Immunohistochemistry and immunofluorescence}

cFos immunohistochemistry was performed on free-floating sections. For peroxidative immunostaining, tissue peroxidases were eliminated with $0.3 \%$ of $\mathrm{H}_{2} \mathrm{O}_{2}$ and methanol $20 \%$, during a period of $30 \mathrm{~min}$. Tissue was incubated for $48 \mathrm{~h}$ with polyclonal primary antibody rabbit anti-cFos (1:1000; Synaptic Systems, Goettingen, Germany) in PBS 0.1M tween X-100 (PBSt) at $4{ }^{\circ} \mathrm{C}$. In a second step, sections were exposed to affinity purified secondary biotinylated antibody goat anti-rabbit (1:400; Jackson InmunoResearch Laboratories, Inc., West Grove, PA, USA) in PBSt for 120 min at room temperature. For magnification, we used preassembled biotin-avidin peroxidase complex according to the Vector Labs recommendations ( $A B C$ Elite; Vector Laboratories, Bulingame, Ca, USA). Sections were exposed to DAB solution with nickel. Then, the tissue was rinsed and mounted in Eukitt (Sigma-Aldrich, Madrid, Spain).

PNNs were labelled by incubating cerebellar tissue with the lectin Wisteria Floribunda Agglutinin (WFA) (1:200; Sigma-Aldrich, Madrid, Spain) at $4{ }^{\circ} \mathrm{C}$ overnight in PBS $0.1 \mathrm{M}$ triton X-100. Samples were then exposed to Cy3-conjugated Streptavidin (1:200; Jackson InmunoResearch Laboratories, Inc., West Grove, PA, USA) for $120 \mathrm{~min}$ at room temperature. The sections were mounted in Mowiol.

\section{Image analysis}

Images from cFos immunostaining were acquired using an optic microscope (Nikon E800, Izasa Werfen Group, Valencia, Spain) with 20x lenses and a resolution of 1,360 x 1,024 dpi. We acquired images of the following regions: the apical region of lobules VIII and IX of the cerebellar vermis, dorsomedial striatum (DMS), dorsolateral striatum (DLS), 
ventrolateral striatum (VLS), NAcC, and NAcSh. Three images were taken by structure and hemisphere in coronal sections. We used bregmas between $1.60 \mathrm{~mm}$ to $0.70 \mathrm{~mm}$ for the striatum and NAc, as well as three sagittal sections between lateral $0.40 \mathrm{~mm}$ to $-0.40 \mathrm{~mm}$ for the cerebellum. Unmanipulated images were used to estimate the density of cFos+ neurons. The estimation was made in selected regions of interest (ROIs) of $80,000 \mu \mathrm{m}^{2}$ for striatal structures and $20,000 \mu \mathrm{m}^{2}$ for the cerebellum. We considered cFos positive neurons only those cells exhibiting a uniform and constant black labelling in the nucleus. Results are given as number of cFos positive neurons per $\mathrm{mm}^{2}$.

PNNs images were captured in a confocal microscope (Leica DMi8, Leica Microsystems CMS GmbH, Wetzlar, Germany) with 20x lenses and resolution 2,048 x 2,048 dpi. Laser intensity (1\%), gain (750), and offset (-1) were maintained constant in each acquisition. Three images in sagittal sections of the dorsal region of lobules VIII and IX were taken from the vermis. We assessed the intensity (brightness range 0-255) of all PNNs in unmodified images by randomly selecting 15 pixels (approximately equidistant from each other) in the net surrounding the neuronal soma and calculating their average. We also counted the number of PNNs and calculated their average manually with ImageJ's software cell counter plugin. We used FIJI free software (Schindelin et al, 2012) for all analyses.

\section{Experimental design and statistics}

Behavioural data relied on preference scores obtained on the test day. Statistical analyses were performed using GraphPad Prism 7 software (GraphPad Software Inc., La Jolla, CA, USA). First, we analysed the effect of mPFC deactivations on preference scores and immunohistochemistry data by means of one-way ANOVAs and post hoc Tukey's HSD tests. Results were presented as mean \pm SEM. Second, we applied an arbitrary cutoff point of $60 \%$ to cluster sham rats in two subgroups: the preference group $(\geq 60 \%$, Sh P) and the no preference group (<60\%, Sh NP) (Gil-Miravet et al, 2018). Our previous findings have shown the utility of such clustering to predict cocaine-induced cerebellar plasticity (Carbo-Gas et al, 2014a, 2014b, 2017) and the effects of mPFC lesions (GilMiravet et al, 2018). Therefore, we compared cFos and PNN expression among the Sham P, Sham NP, mPFC deactivation (PL or IL) and Unp groups. Comparisons between variances and frequencies in these groups were carried out using Kruskal-Wallis for non- 
parametric distributions. Post hoc analyses were performed by Dunn's multiple comparison tests. Spearman's correlation analyses were calculated to investigate the correlation between cFos expression and preference scores. The statistical level of significance was set at $p<0.05$. Scatterplots were depicted in all figures.

\section{RESULTS}

Histological infusion sites and their respective diffusion areas can be seen in figure 1. The comparison of preference scores between the sham IL and sham PL groups did not yield significant differences (Student's t-tests $(t(12)=0.06335, P=0.9505)$. For this reason, we pooled both groups to form a single sham group for the analysis. Then, the sham group was split using the cut-off point of $60 \%$ into the preference (Sh P) and no preference (Sh NP) groups.

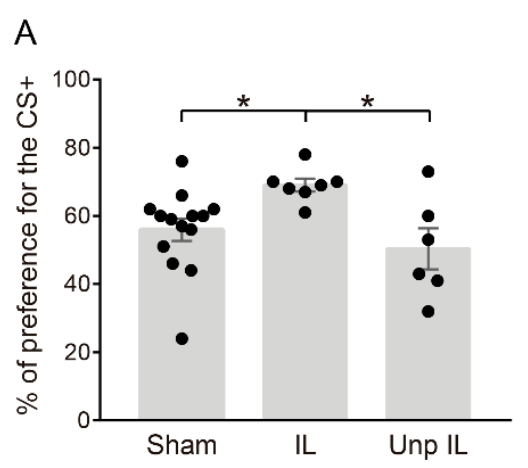

C

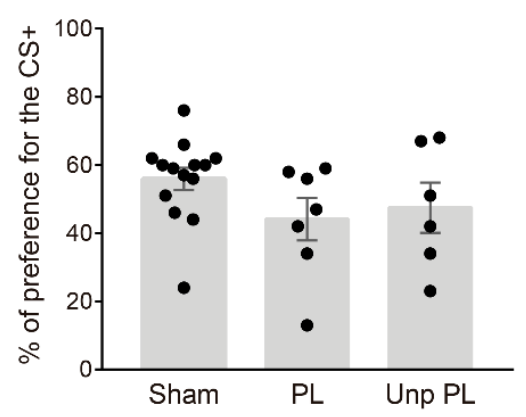

B

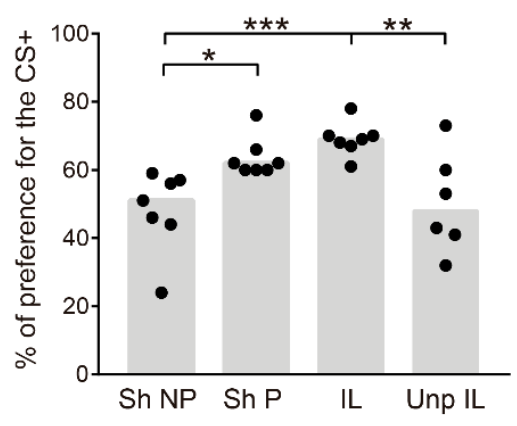

D

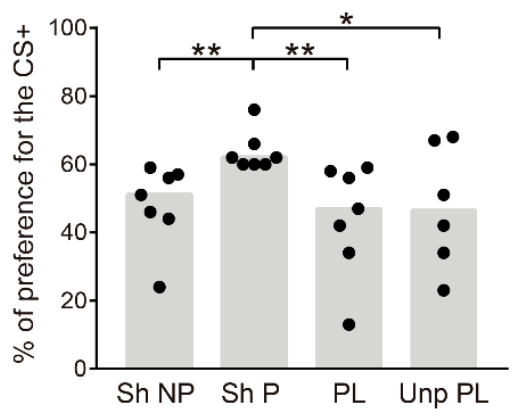

Figure 2. Effect of IL or PL temporal deactivation before each training session on cocaine-induced conditioned preference The $I L$ deactivation increased the proportion of rats that expressed cocaineinduced conditioned preference, while the PL deactivation prevented it. (A) IL deactivations. Data are shown as the mean \pm SEM and scatterplots of the preference scores for the CS+ on the test day in the sham $(n=14)$, infralimbic deactivation (IL) ( $n=7)$ and unpaired IL deactivation (Unp IL) ( $n=6)$ groups. (C) PL deactivations. Mean \pm SEM and scatterplots of preference scores for the CS+ on the test day in the sham $(n=14)$, prelimbic deactivation $(P L)(n=7)$ and unpaired $P L$ deactivation (Unp $P L)(n=6)$ groups. (B-D) Results after clustering the sham group in the sham no preference (Sh NP) $(n=7)$ and sham preference (Sh $P)(n=7)$ groups. Data are shown as the median and scatterplots. $\left({ }^{*} P<0.05 ;{ }^{* *} P<0.01 ; * * P<0.001\right)$. 
A one-way ANOVA showed significant effects for the group factor $(F(2,24)=4.774, p=$ 0.0180). As shown by a subsequent post hoc comparison using Tukey's HSD tests, the IL deactivation facilitated the acquisition of cocaine-induced preference conditioning. The IL group $(n=7)$ exhibited a significant higher preference for the CS+ than the sham $(n=$ 14) $(p=0.049)$ and unpaired groups (Unp IL) $(n=6)(p=0.019)$ (Fig. 2A). On the contrary, the PL deactivation did not produce a significant effect on cocaine-induced conditioned preference $(F(2,24)=1.718, p=0.2007)$ (Fig. $2 C)$. No lateralisation effect was found in any of the groups when comparing left and right deactivation sides: Sham $(t)(12)=$ $0.5654, \mathrm{P}=0.5822) ; \mathrm{IL}(\mathrm{t}(5)=1.076, \mathrm{P}=0.3310) ; \mathrm{PL}(\mathrm{t}(5)=0.0655, \mathrm{P}=0.9503)$.

After clustering rats using the cut-off point for preference (60\%), a Kruskal-Wallis analysis confirmed that IL deactivation increased the number of animals that exhibited a preference score higher than $60 \%(H(4)=15.66, P=0.0013)$ (Fig. $2 B)$. Dunn's post hoc comparisons showed that the IL group $(n=7)$ exhibited significantly higher preference for the CS+ than the Sh NP $(n=7)(P=0.0005)$ and Unp IL $(n=6)(P=0.0042)$ groups. However, post hoc comparisons revealed no differences between the IL and Sh P groups $(P>0.2)$ (Fig. 2B).

Oppositely, PL deactivation seemed to block the acquisition of cocaine-induced preference conditioning. A Kruskal-Wallis test showed a significant effect of the group factor $(H(4)=10.71, P=0.0134)$, being the Sh $P$ group that exhibited a higher preference as compared to the Sh NP $(P=0.0081), P L(P=0.0033)$ and Unp PL $(P=0.0278)$ groups (Figure 2D).

\section{Deactivations of mPFC generate differential effects on cerebellar activity}

We focused our analysis on cFos activity in the apical (dorsal) region of lobules VIII and IX, as previous research from the group indicated that this is the cerebellar region that showed significant differences linked to the expression of cocaine-induced conditioned preference (Carbo-Gas et al, 2014a, 2014b). Therefore, we were interested in investigating whether the effects of MPFC deactivations on cerebellar activity could be modulated by the expression of preference for the cocaine-related cue. A Kruskal-Wallis test showed significant effects in the number of cFos+ cells in lobule VIII $(H(4)=22.01$, 

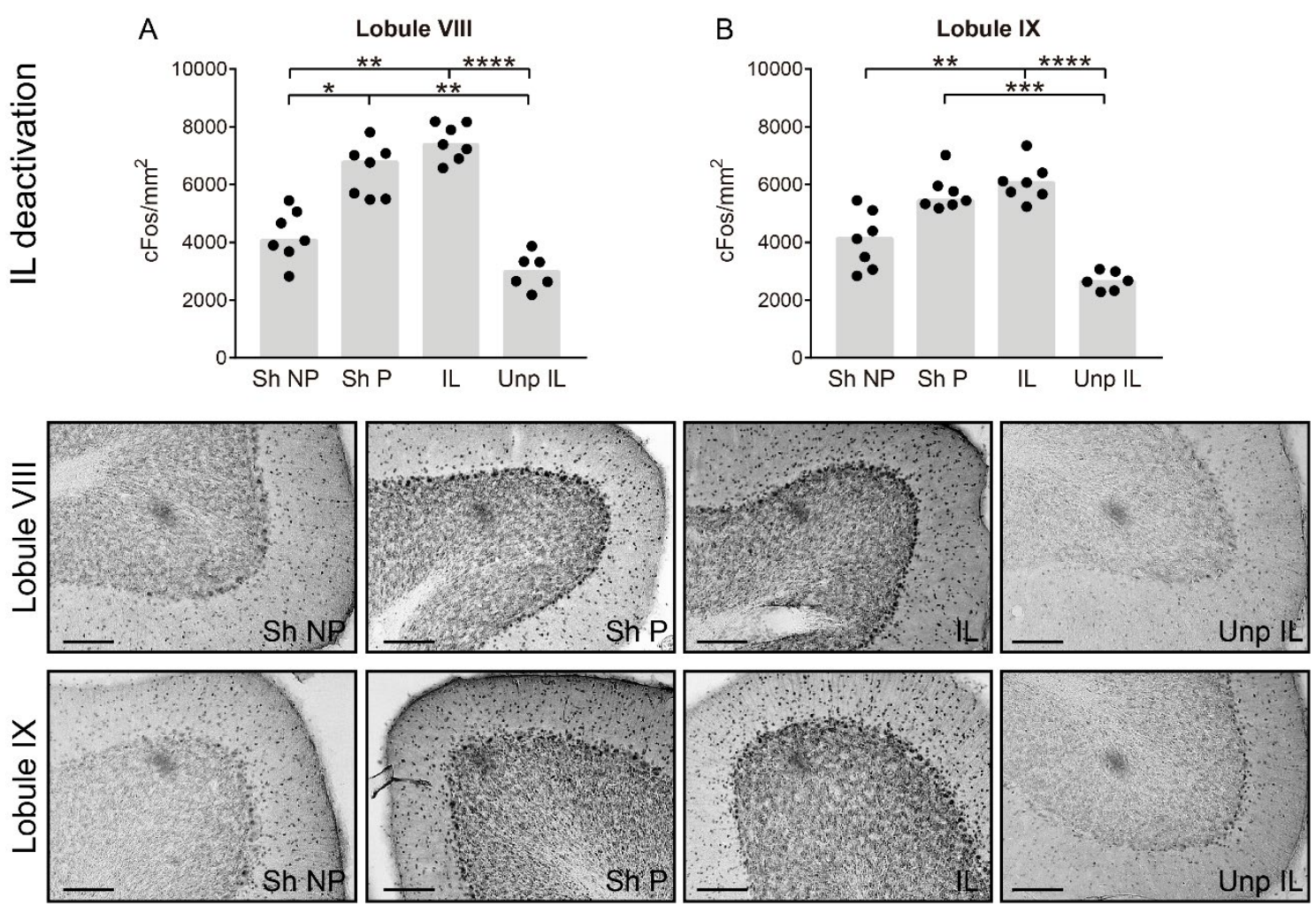

Figure 3. Effect of PL deactivation on cFos expression in the dorsal region of lobules VIII and IX of the cerebellar vermis. (A-B) Number of cFos positive cells $/ \mathrm{mm}^{2}$ in lobule VIII and IX 90 min after the preference test in the Sh NP $(n=7)$, Sh $P(n=7)$, prelimbic deactivation $(P L)(n=7)$ and unpaired $P L$ deactivation (Unp $P L)(n=6)$ groups. Representative pictures of cFos staining in lobule VIII and IX for each group are depicted on the bottom panel. All images were taken at $20 x$ magnification. Scale $50 \mu m .\left({ }^{*} P<0.05 ;{ }^{* *} P<0.01\right.$; $* * * P<0.001, * * * * P<0.0001)$. Brightness was standardised across images.

$\mathrm{P}<0.0001)$ and lobule IX (H (4) = 19.99, $\mathrm{P}=0.0002)$. In lobule VIII, cFos expression increased in the IL and Sh P groups regarding the groups not expressing preference (Sh NP: $P=0.0017$ and $P=0.0368$, respectively) and (Unp IL: $P<0.0001$ and $P=0.0014$, respectively) (Fig. 3A). Similar increase in the number of cFos+ neurons was found in lobule IX as an effect of the IL deactivations (Sh NP $(P=0.0071)$ and Unp IL $(P<0.0001)$ groups). However, in this case, the Sh $\mathrm{P}$ group was only different from the Unp IL (P = 0.0010) group (Fig. 3B).

The expression of cFos in the cerebellum after PL deactivations showed a different pattern. Kruskal-Wallis test also demonstrated a significant effect for lobule VIII $(H(4)=$ 18.52, $P=0.0003)$ and $I X(H(4)=18.35, P=0.0004)$. However, Dunn's multiple comparisons test for lobule VIII revealed that the number of cFos+ neurons in the PL group was not different from that of the Sh NP $(P>0.3)$ and Unp PL $(P>0.3)$ groups. Only the Sh P group exhibited higher cFos expression than the other groups (Sh NP (P = 0.022), PL ( $P=0.0016)$, Unp PL $(P<0.0001)$ ) (Fig. 4A). Similar results were observed for 

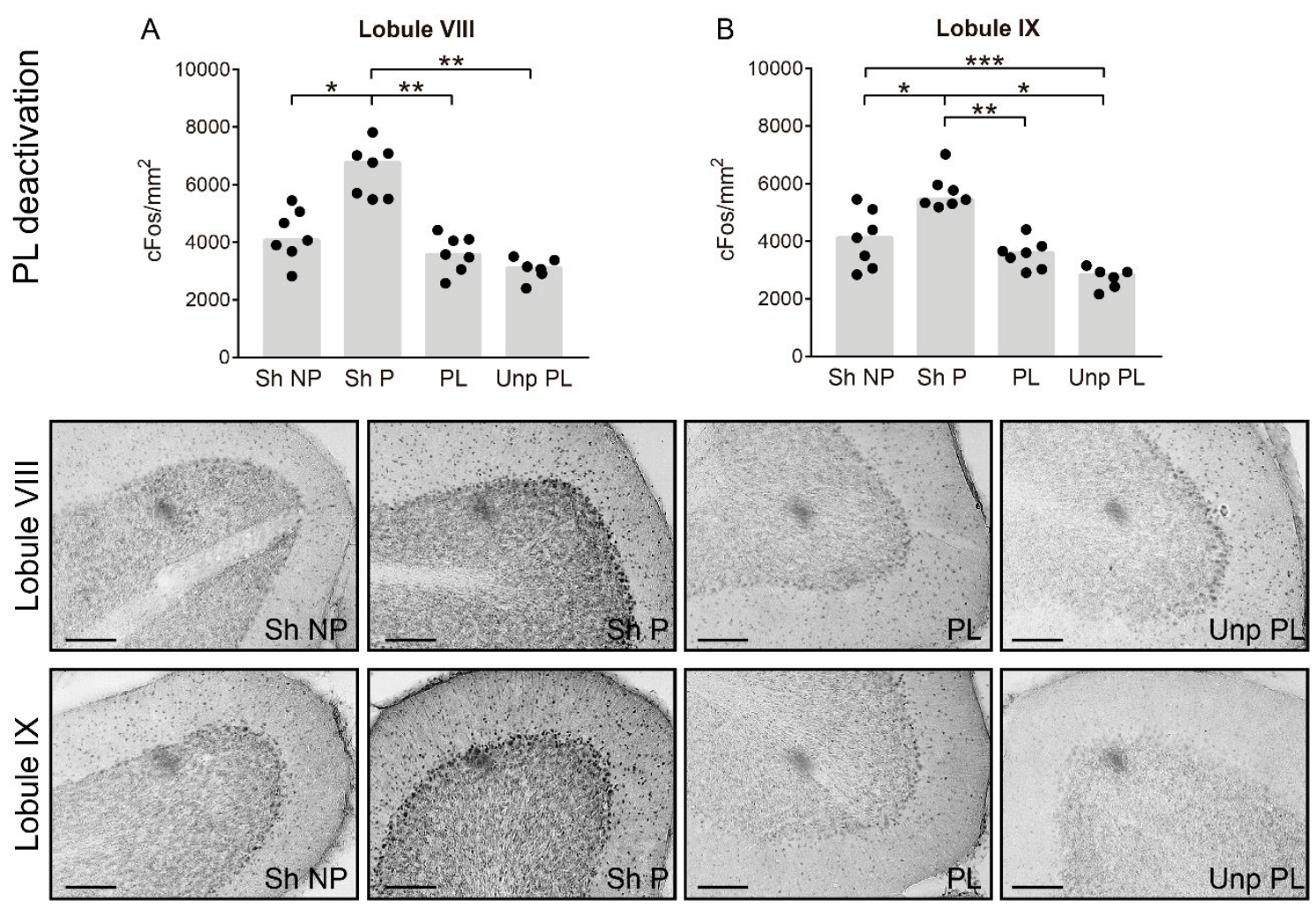

Figure 4. Effect of IL deactivation on cFos expression in the dorsal region of lobules VIII and IX of the cerebellar vermis. (A-B) Number of cFos positive cells $/ \mathrm{mm}^{2}$ in lobule VIII and IX, 90 min after the preference test in the Sh NP $(n=7)$, Sh $P(n=7)$, IL deactivation $(n=7)$ and Unp IL deactivation $(n=6)$ groups. Representative pictures of cFos staining in lobule VIII and IX for each experimental group appear below. All images were taken at $20 x$ magnification. Scale bar $50 \mu m .\left(^{*} P<0.05 ;{ }^{* *} p<0.01 ; * * *<0.001, * * * * P\right.$ $<0.0001)$. Brightness was standardised across images.

lobule IX. The expression of cocaine-induced preference (Sh P) increased cFos levels as compared to those groups that did not express preference (Sh NP $(P=0.0339), P L(P=$ 0.0071), Unp PL (P<0.0001)) (Fig. 4B).

Significant correlations were A observed between cFos expression and preference in lobule VIII $(r=0.8216 ; P<0.0001)$ and IX ( $r=0.7956 ; P<0.0001)$ (Fig. 5A-B).
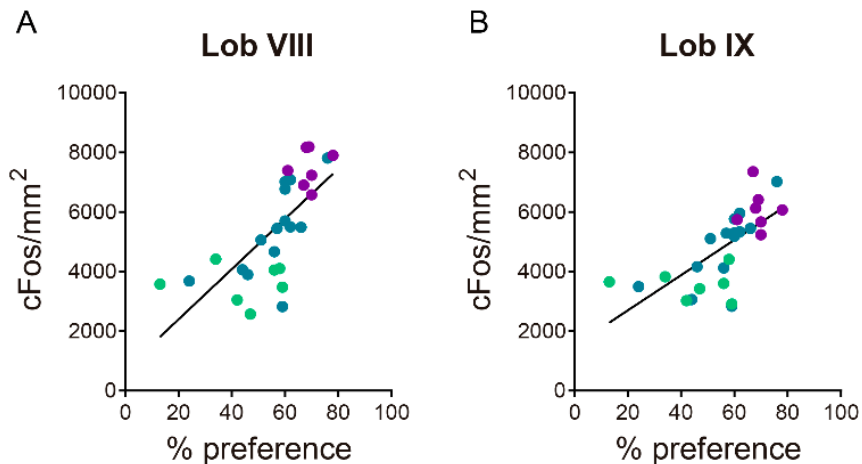

In summary, the expression of preference for cocaine-related cues was associated with an Figure 5. Correlations between cFos expression and preference scores in lobules VIII and IX (A) Correlation between cFos positive cells $/ \mathrm{mm}^{2}$ and percentage of preference in lobule VIII. (B) Correlation between cFos positive cells $/ \mathrm{mm}^{2}$ and preference percentage in lobule. IL group (purple dots), PL group (green increase in cFos expression in the dots), sham group (blue dots). 
posterior cerebellum. IL and PL deactivations oppositely influenced cerebellar activity, and their effects seem to be modulated by the expression of cocaine-induced conditioned preference. While all rats with IL deactivation showed conditioned preference and an increase in cerebellar activity, those that underwent PL deactivation did not.

\section{Different impact of mPFC deactivations on striatal activity}

The Kruskal-Wallis tests for the results of the IL experiment showed significant effects on cFos expression in the DMS $(H(3)=7.75, P=0.0208)$ and NAcSh $(H(3)=12.79, P=$ $0.0017))$, but not in the DLS $(H(3)=2.08, P=0.3539), \operatorname{VLS}(H(3)=2.57, P=0.2773)$, and $\mathrm{NAcC}(\mathrm{H}(3)=1.21, \mathrm{P}=0.5672)$ (Fig. $6 \mathrm{~A})$. Significantly greater numbers of cFos+ neurons were observed in the DMS and NAcSh for the IL $(P=0.0264, P=0.0071)$ and Sh $P(P=$ $0.0103 ; P=0.0007)$ groups as compared to the Sh NP group, respectively.

Significant effects were observed in the DMS $(H(3)=7.03, P=0.0 .297), N A c C(H(3)=$ 9.11, $\mathrm{P}=0.0 .105)$ and NAcSh $(\mathrm{H}(3)=11.26, \mathrm{P}=0.0011)$, but not in the DLS $(\mathrm{H}(3)=1.79$, $P=0.4077)$ and VLS (H (3) = 3.29, $P=0.1932)$ in the PL experiment (Fig. 6B). For the DMS,
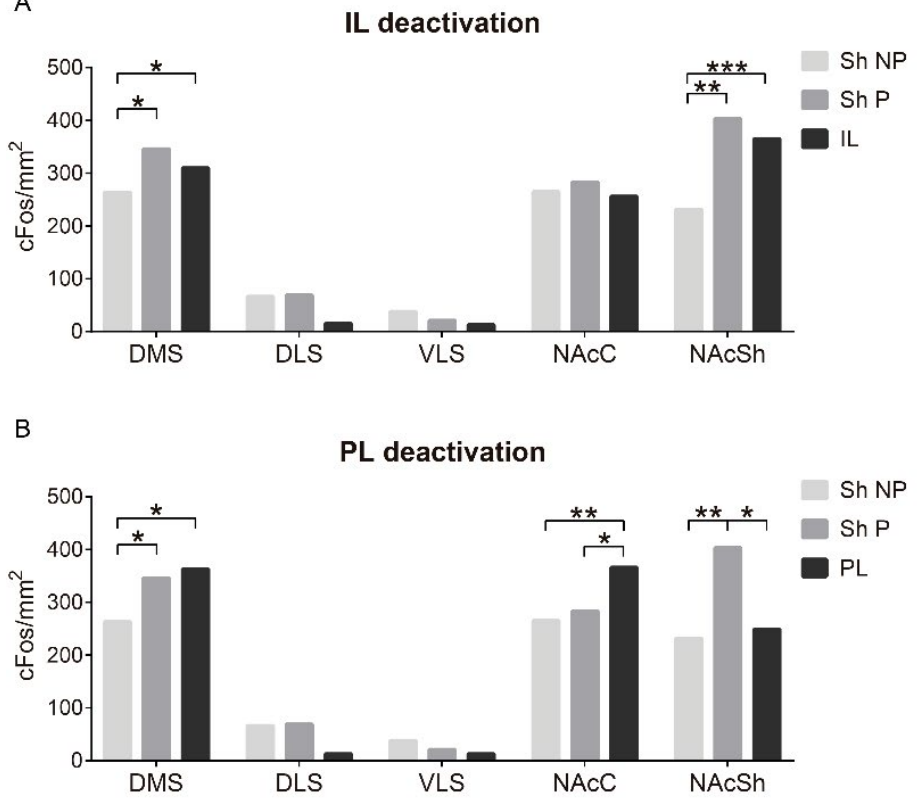

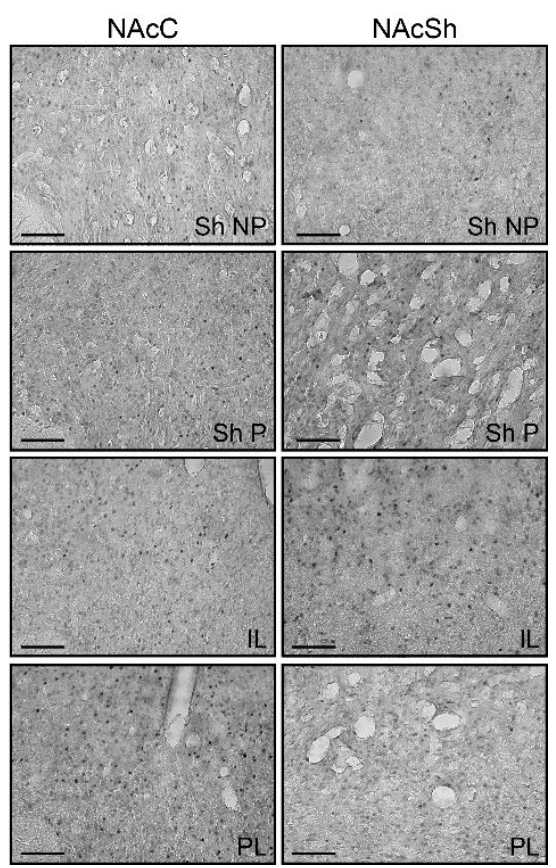

Figure 6. Mean number of cFos positive cells/mm2 in DMS, DLS, VLS, NAcC and NAcSh for IL and PL deactivations. (A) IL deactivation. DMS, DLS, VLS, NAcC and NAcSh in Sh NP $(n=7)$, Sh $P(n=7)$ and IL $(n=$ 7) groups. (B) PL deactivation. DMS, DLS, VLS, NAcC and NAcSh in Sh NP $(n=7)$, Sh $P(n=7)$ and $P L(n=7)$ groups. On the right, representative pictures of CFos staining in the NACC and NAcSh for the four experimental groups. All images were taken at $20 x$ magnification. Scale bar $50 \mu m$. $\left({ }^{*} P<0.05 ; * * P<0.01\right.$; $* * * P<0.001)$. Brightness was standardised across images. Data are shown as the median score 
the Sh NP group showed lower cFos activity than the Sh $P(P=0.0251)$ and $P L(P=0.0189)$ groups. In the NAcC, PL deactivation increased the number of cFost neurons as compared to the Sh NP $(P=0.0032)$ and Sh $P(P=0.0429)$ groups. Finally, in the NAcSh, the level of cFos expression in PL deactivated animals was very similar to that of the Sh NP group. Only the Sh P group showed a significant increase in cFos activity with respect to the other groups (Sh NP: $\mathrm{P}=0.0014 ; \mathrm{PL}: \mathrm{P}=0.0125$ ).

Taken together, the present results indicated that mPFC deactivations showed differential effects in the NAc. After IL deactivation, activity in the NAcSh increased at the same level as that of those animals expressing preference. On the contrary, PL deactivation increased neural activity within the NACC, but did not change activity in the NAcSh. Moreover, after the PL deactivation, neural activity in the NAcSh was very similar to that exhibited by those rats not expressing preference for cocaine-related cues. Deactivation of both MPFC regions impacted DMS activity similarly and did not change neural activity in other striatal regions.

\section{Cocaine-induced conditioned preference upregulates PNN expression around Golgi interneurons in the cerebellar cortex}

The expression of PNNs surrounding Golgi interneurons showed significant effects for the group factor in both experiments. (IL) lobule VIII (H (4) = 19.87, P = 0.0002) and IX (H $(4)=19.58, P=0.0002) ;(P L)$ lobule VIII $(H(4)=12.25, P=0.0066)$, and $I X(H(4)=14.69$, $P=0.0021)$.

Cerebellar PNNs in those groups expressing preference (IL and Sh P) were stronger than PNNs in the Sh NP $(P=0.0011 ; P=0.0184$, respectively $)$ and Unp IL $(P=0.0002$ and $P=$ 0.0039 , respectively) groups (Fig. 7A). In Lobule IX, the results were very similar, and more intense PNNs were found in the IL and Sh P groups as compared to the Sh NP $(P=$ 0.0005; $P=0.0311$, respectively) and Unp IL $(P=0.0002 ; P=0.0125$, respectively) groups (Fig. 7B).

Oppositely, PL deactivation induced fainter expression of PNNs in lobules VIII and IX resembling the expression of those groups that did not express cocaine-induced conditioned preference. Thus, in this case, only the Sh P group showed PNNs more intense than the rest of groups in lobule VIII $(P<0.02)$ and IX $(P<0.05)$ (Fig. 8A-B). 
A
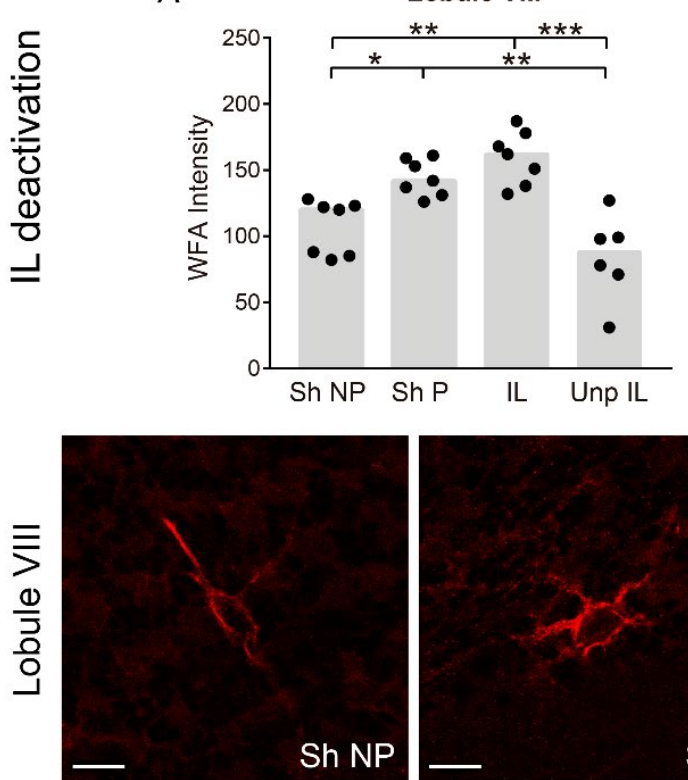

$\frac{x}{\frac{0}{7}}$

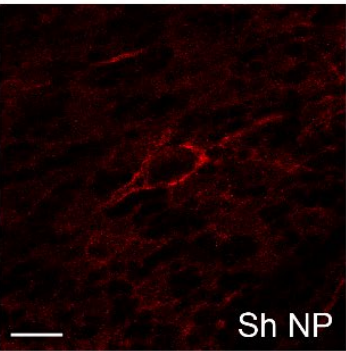

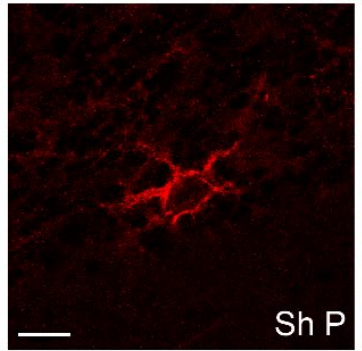

B
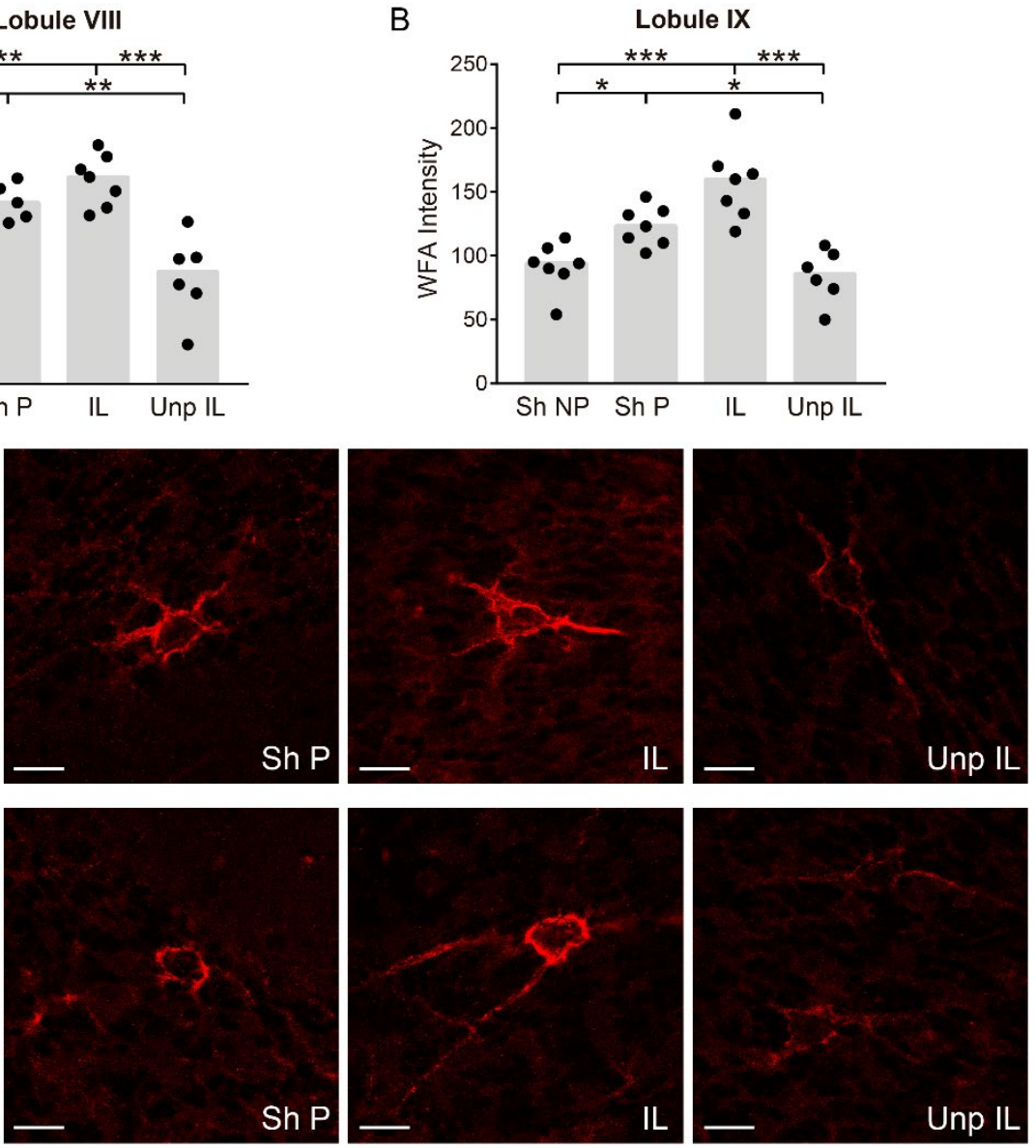

Figure 7. PNN expression surrounding Golgi interneurons in the posterior vermis after IL deactivation. $(A-B)$ Average intensity of WFA at the dorsal region of the granule cell layer in lobules VIII and IX in the Sh $N P(n=7)$, Sh $P(n=7), I L(n=7)$ and Unp $I L(n=6)$ groups. On bottom panels, representative microphotographs of PNNs around Golgi interneurons stained with Wisteria floribunda agglutinin (WFA) (red). The confocal images were acquired at $20 x$ with a $2 x$ zoom for a final amplification of $80 x$, respectively. Scale bar $20 \mu \mathrm{m}$. PNN surrounding Golgi cells were still stronger and more prominent in the Sh $P$ and IL groups. $\left({ }^{*} P<0.05 ;{ }^{*} P<0.01 ;{ }^{* *} P<0.001\right)$.

Finally, the correlation between preference and PNNs intensity were significant in lobule VIII ( $r=0.6521 ; P=0.0002)$ and in the lobule IX ( $r=0.5817 ; P=0.0012)$ (Fig. 9A-B).

In conclusion, the expression of preference for the cocaine-related cue was associated with stronger and fully condense PNNs. As was previously described for neural activity, the key factor in explaining the upregulation of PNNs around Golgi interneurons was the acquisition of cocaine-induced conditioned memory that seemed to be mediating the effects of mPFC deactivations on cerebellar plasticity. 

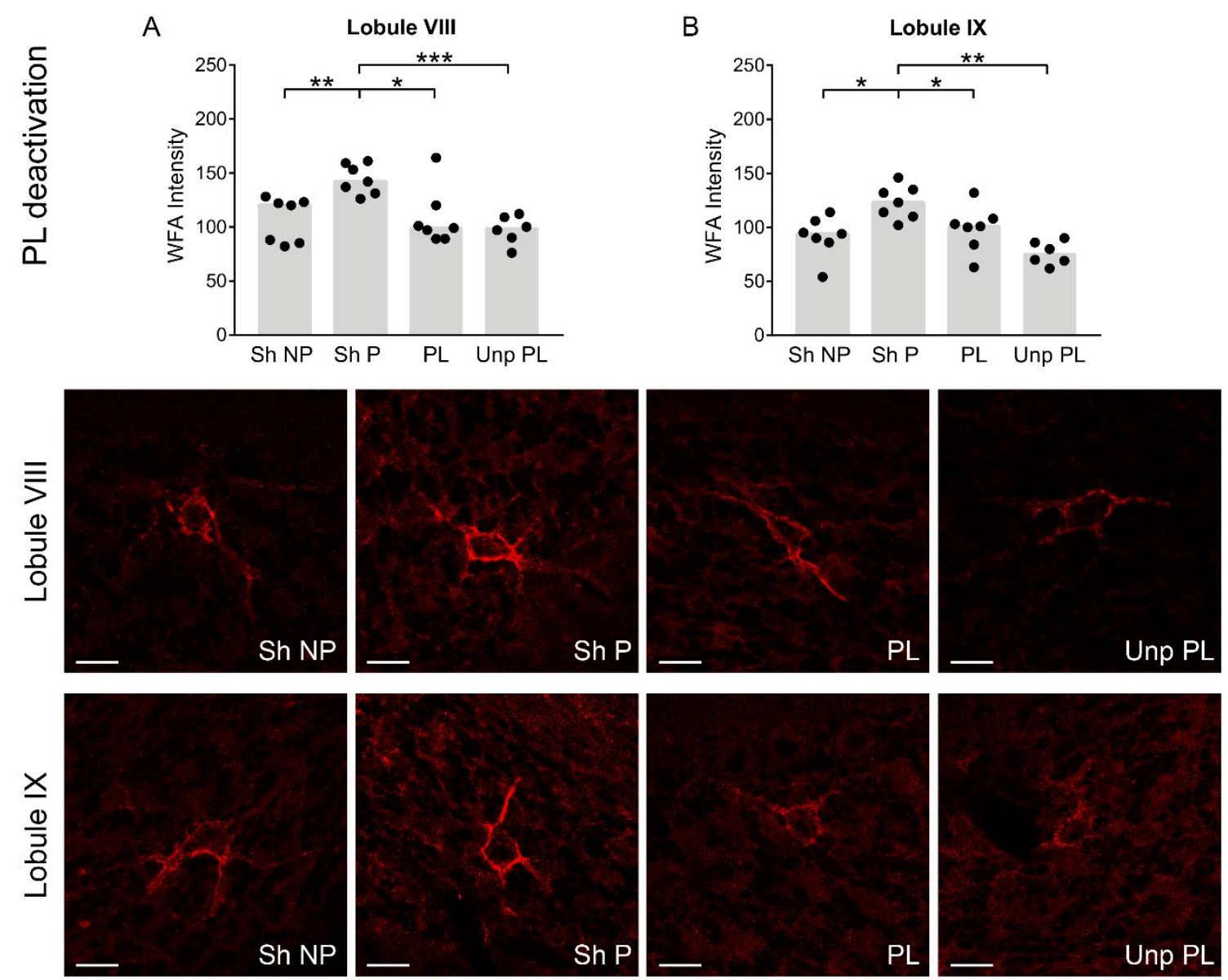

Figure 8. PNN expression around Golgi interneurons the posterior vermis after PL deactivations. (A-B) Average intensity of WFA at the dorsal region of the granule cell layer in lobules VIII in the Sh NP $(n=7)$, Sh $P(n=7), P L(n=7)$ Unp PL $(n=6)$ groups. Representative microphotographs of PNNs in the dorsal region of cerebellar cortex stained with Wisteria floribunda agglutinin (WFA) (red). The confocal images were acquired at 20x with a $2 x$ zoom for a final amplification of $80 x$, respectively. Scale bar $20 \mu m$. PNN surrounding Golgi cells were stronger and more prominent only in the Sh P group. $\left(* P<0.05 ;{ }^{*} P<0.01\right.$; $* * * P<0.001)$.
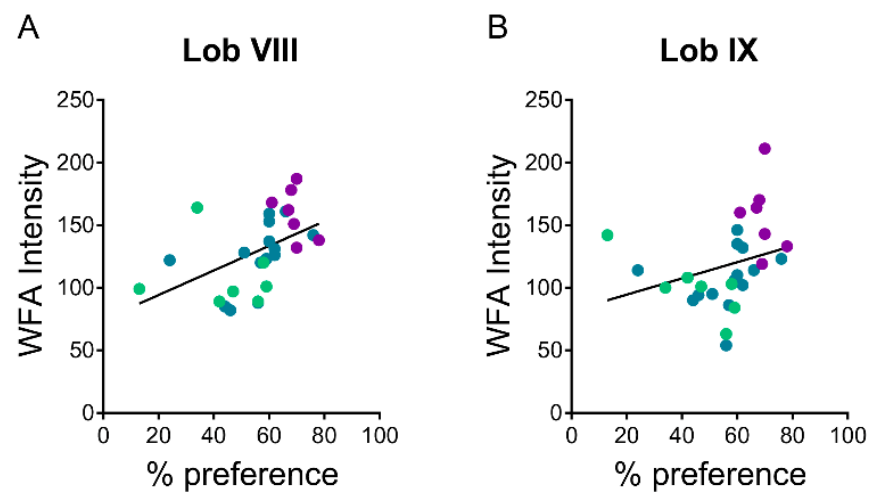

Figure 9. Correlations between PNNs intensity and preference scores in lobules VIII and IX (A) Correlation between WFA intensity and percentage of preference in lobule VIII. (B) Correlation WFA intensity and preference percentage in lobule IX. IL group (purple dots), PL group (green dots), sham group (blue dots). 


\section{DISCUSSION}

A large number of studies have shown that prefrontal cortex plays a crucial role in drug addiction in animals and humans (Chen et al, 2013; Goldstein and Volkow, 2011; Lasseter et al, 2010; McFarland and Kalivas, 2001; Volkow et al, 2015). Functional impairment of the prefrontal cortex underlies loss of inhibitory control over drugseeking (Chen et al, 2013; Jentsch and Taylor, 1999). Remarkably, the cerebellum, always being considered a motor structure, has revealed itself as an important region for the addiction field during the last decade (Miquel et al, 2009, 2016; Moulton et al, 2014). In previous studies, we showed that cocaine-induced preference conditioning enhanced cFos expression and upregulates PNN expression in the apical region of the posterior cerebellum in mice (Carbo-Gas et al, 2014a, 2014b, 2017). In the present study, we wanted to explore whether MPFC hypofunction would influence cocainedependent activity and plasticity in the cerebellum. Thus, we trained rats to acquire a cocaine-cue conditioned association under temporal deactivation of either the IL or PL cortex. Our findings indicated that impairment of these two regions in the MPFC may have a different impact on cocaine-related memory. Then, such an impact modulates neuronal activity and plasticity in the cerebellum.

In the first chapter (Gil-Miravet et al, 2018), we demonstrated that an IL deactivation produces a robust increase in the percentage of animals that develop a preference for the cocaine-related cue. The analysis of the subsample of rats we selected for the immunohistochemistry study yielded the same results. Therefore, we can conclude that IL deactivation during conditioning increased the likelihood of acquiring drug-induced memories. As lidocaine administrations were performed before each training session, but not on the test day, the IL deactivation could only affect the acquisition phase. In this way, on the test day, IL deactivated animals were under similar conditions to those in the sham group. Our results, together with the already existing literature (Lalumiere et al, 2012; LaLumiere et al, 2010; Peters et al, 2008; Rocha and Kalivas, 2010), suggest that the IL cortex can be part of an inhibitory route for the acquisition of drug-seeking.

IL deactivation enhanced neural activity in the apical region of the granule cell layer in the posterior vermis. This increase was not seen in animals with PL deactivation. Neither was it seen in those cerebella from control animals that did not express preference for 
the cocaine-related cue. However, a more careful analysis of the present data did not support a direct effect of the IL deactivation. Neural activity in this region of the cerebellar cortex also increased in control rats expressing preference. Hence, our results suggest that IL deactivation by facilitating the acquisition of cocaine-induced conditioned preference increased neural activity in the cerebellum. The present results are consistent with our previous observations in mice and support also our hypothesis about the function of the posterior cerebellar cortex in drug reward (Carbo-Gas et al, 2014a, 2014b, 2017; Moreno-Rius and Miquel, 2017). We have proposed that the posterior cerebellar cortex generates unconscious predictions of drug availability after cue presentation while monitoring the internal state under drug abstinence (MorenoRius and Miquel, 2017). Cue exposure would then trigger a cerebellum-generated prediction of drug availability, thereby activating the preparation of the brain networks (striatal-limbic loops) responsible for drug-seeking and taking. Moreover, we observed that when animals are confined in the only presence of the cocaine-related cue without the possibility of selecting the other alternative (CS-), the increase in cerebellar activity is prevented (Carbo-Gas et al, 2017). Therefore, our past and present results indicate that neural activity in the granule cell layer of the cerebellar cortex may reflect the behavioural selection driven by the cocaine-related cue. They also indicate that if drug experience occurs under IL impairment, the probability for the acquisition of cocaineinduced conditioned memory, as well as the development of associated cerebellar changes raises. Interestingly, neuroimaging studies showed that greater cerebellar activity accompanied prefrontal dysfunction in drug addicts (Bolla et al, 2005; Desmond et al, 2003; Hester and Garavan, 2004; Moulton et al, 2014).

In the present research, the acquisition of cocaine-induced preference was also associated with an upregulation of PNNs around Golgi inhibitory interneurons in the apical region of the cerebellar cortex. Stronger and fully condense PNNs were found after IL deactivation, but also in control animals that acquired the conditioned memory. These findings suggest that cocaine-induced conditioned memory, and not IL hypofunction, is the key factor in increasing PNNs expression around Golgi interneurons. Indeed, those groups that did not express conditioned preference, such as the PL deactivation group, pseudo-conditioned groups, and the no preference group all exhibit 
faint PNNs around Golgi interneurons. Similar results were described previously in mice (Carbo-Gas et al, 2017). The expression of PNNs surrounding Golgi inhibitory interneurons in the apical region of the cerebellar cortex were stronger in animals that exhibit preference for the cue associated with cocaine. A reduced PNN intensity might correspond to an immature PNN with increased capacity for plasticity, whereas higher intensity would correspond to a mature PNN with decreased capacity for plasticity (Wang and Fawcett, 2012). In this way, the animals that acquired preference and, therefore, generated cocaine associated memories, developed fully condense PNNs and consequently their capacity for plasticity was reduced. Sorg et al. (2016) have proposed that stronger PNNs could "stamp in" synaptic connections that represent drug-cue associations, preventing future synaptic remodelling. With stronger PNNs, the new synapses formed during drug-induced conditioning would be more stable and difficult to modify. Accordingly, a recent study observed that the degradation of PNNs in the PL cortex in cocaine-induced CPP memory impaired acquisition and reconsolidation of cocaine-induced place preference memories (Slaker et al, 2015). Moreover, PNNs degradation in the amygdala following drug exposure, but before extinction training, augments extinction and inhibits subsequent reinstatement of drug-seeking behaviour (Xue et al, 2014). Other recent investigations have observed that the degradation of PNNs in the lateral hypothalamic area abolishes the acquisition of cocaine-induced conditioned place preference, reduces cocaine self-administration, and blocked the expression of cue-induced reinstatement of cocaine- but not sucrose-seeking behaviour (Blacktop et al, 2017; Blacktop and Sorg, 2018).

In the cerebellum, PNNs are also developed around glutamatergic and GABAergic projection in the deep cerebellar nuclei (DCN) (Carulli et al, 2006). These PNNs did not change after the acquisition of cocaine-induced preference memory, at least as far as the medial nucleus concerns (Carbo-Gas et al, 2017). They have demonstrated, however, to be regulated by plasticity events affecting Purkinje neurons during periods of cocaine abstinence. Thus, stronger and more intense PNNs are found after a short period of abstinence associated with a kind of molecular and structural plasticity which reduce Purkinje cell capacity to inhibit DCN neurons (Vazquez-Sanroman et al, 2015). On the contrary, PNNs are downregulated in the medial nucleus when the synaptic capacity 
of Purkinje neurons increased after longer periods of abstinence (Vazquez-Sanroman et al, 2015).

Deactivations of the IL and PL cortices generated different effects on activity in the NAc. Literature suggests that the PL-to-NACC pathway promotes drug-seeking behaviour and the IL-to-NAcSh pathway is responsible for the extinction of drug-seeking behaviour (Lalumiere et al, 2012; McFarland and Kalivas, 2001; Peters et al, 2008). As observed in the cerebellum, neural activity in the NAcSh was related to cocaine-induced conditioned preference. NAcSh activity increased only in animals expressing preference. Several studies suggest that NAcSh is involved in the suppression of cocaine-seeking behaviour (Di Ciano et al, 2008; Peters et al, 2008). Lalumiere et al. (2012) found that AMPA receptor blockade in the NAcSh restored cocaine-seeking previously inhibited by a positive modulator of AMPA receptor activation of IL afferents to the NAcSh (Lalumiere et al, 2012). Oppositely, AMPA receptor blockade in the NAcC prevented cue-induced reinstatement of cocaine-seeking (Bäckström and Hyytiä, 2007; Di Ciano and Everitt, 2001; Cornish and Kalivas, 2000; McFarland and Kalivas, 2001). The NAcSh and NAcC receive glutamatergic input from the $\mathrm{IL}$ and $\mathrm{PL}$, respectively, and dopaminergic inputs from the VTA (Fallon and Moore, 1978; Sesack et al, 1989). A recent study observed that optical inhibition of VTA-to-NAcC afferents prevented cocaine-seeking reinstatement (Stefanik and Kalivas, 2013). These dopaminergic projections seem to be critical for learning and maintenance of goal-directed responding, including drug-seeking behaviour (Ettenberg, 1989; Koob and Swerdlow, 1988). Moreover, dopaminergic and non-dopaminergic projections from the VTA to cerebellum have been described (Ikai et $a l, 1992,1994)$, supporting the presence of dopamine transporters and receptors in the cerebellar cortex and deep nuclei (Ikai et al, 1992; Melchitzky and Lewis, 2000; Panagopoulos et al, 1991). Previous studies in our laboratory observed increases in levels of dopamine transporter in the posterior cerebellum of animals that develop cocaine-induced conditioned preference (Carbo-Gas et al, 2014a).

In conclusion, our laboratory studies do not support a direct effect of mPFC deactivations on cerebellar activity and plasticity. On the contrary, they indicate that impairment of the MPFC function increases or decrease susceptibility to the acquisition of drug-induced Pavlovian memory thereby modulating cerebellar activity. In addition, 
they suggest that the cerebellum might be a critical region for the storage or reactivation of conditioned associations that predict drug availability.

\section{ACKNOWLEDGMENTS}

This research was supported by the following grants and fellowships: Ministerio de Educación Cultura y Deporte (FPU12/04059); Universitat Jaume I (PREDOC2014/11); UJ (14I307.01/1); Ministerio de Economía y Competitividad (MINECO) (PSI2015-68600-P); and Plan Nacional de Drogas 2017 (PND-132400).

\section{AUTHOR DISCLOSURE}

All authors declare no conflicts of interest.

\section{AUTHORSHIP}

All authors made a notable contribution to the manuscript, and they were involved in critically revising the present version. Isis Gil-Miravet performed the stereotaxic surgeries and behavioural experiments. Isis Gil-Miravet and Ignasi Melchor-Eixea were involved in image and data analysis. Finally, Marta Miquel designed the study, supervised the surgeries and behavioural experiments, was involved in data analysis, and drafted the manuscript. All authors approved the present version of the manuscript.

\section{DATA ACCESSIBILITY}

Raw data are available from the corresponding author upon request. 


\section{REFERENCES}

Anderson CM, Maas LC, Frederick B, Bendor JT, Spencer TJ, Livni E, et al (2006). Cerebellar Vermis Involvement in Cocaine-Related Behaviors. Neuropsychopharmacology 31: 1318-1326.

Bäckström P, Hyytiä P (2007). Involvement of AMPA/kainate, NMDA, and mGlu5 receptors in the nucleus accumbens core in cue-induced reinstatement of cocaine seeking in rats. Psychopharmacology (Berl) 192: 571-80.

Ball KT, Slane M (2012). Differential involvement of prelimbic and infralimbic medial prefrontal cortex in discrete cue-induced reinstatement of 3,4methylenedioxymethamphetamine (MDMA; ecstasy) seeking in rats. Psychopharmacology (Berl) 224: 377-85.

Blacktop JM, Sorg BA (2018). Perineuronal nets in the lateral hypothalamus area regulate cue-induced reinstatement of cocaine-seeking behavior. Neuropsychopharmacology 1-9doi:10.1038/s41386-018-0212-8.

Blacktop JM, Todd RP, Sorg BA (2017). Role of perineuronal nets in the anterior dorsal lateral hypothalamic area in the acquisition of cocaine-induced conditioned place preference and self-administration. Neuropharmacology 118: 124-136.

Bolla KI, Eldreth DA, Matochik JA, Cadet JL (2005). Neural substrates of faulty decisionmaking in abstinent marijuana users. Neuroimage 26: 480-492.

Bostan AC, Strick PL (2018). The basal ganglia and the cerebellum: nodes in an integrated network. Nat Rev Neurosci 19: 338-350.

Capriles N, Rodaros D, Sorge RE, Stewart J (2003). A role for the prefrontal cortex in stress- and cocaine-induced reinstatement of cocaine seeking in rats. Psychopharmacology (Berl) 168: 66-74.

Carbo-Gas M, Moreno-Rius J, Guarque-Chabrera J, Vazquez-Sanroman D, Gil-Miravet I, Carulli D, et al (2017). Cerebellar perineuronal nets in cocaine-induced pavlovian memory: Site matters. Neuropharmacology 125: 166-180.

Carbo-Gas M, Vazquez-Sanroman D, Aguirre-Manzo L, Coria-Avila GA, Manzo J, SanchisSegura C, et al (2014a). Involving the cerebellum in cocaine-induced memory: Pattern of cFos expression in mice trained to acquire conditioned preference for cocaine. Addict Biol 19: 61-76.

Carbo-Gas M, Vazquez-Sanroman D, Gil-Miravet I, las Heras-Chanes J De, Coria-Avila GA, Manzo J, et al (2014b). Cerebellar hallmarks of conditioned preference for cocaine. Physiol Behav 132: 24-35.

Cardinal RN, Parkinson JA, Hall J, Everitt BJ (2002). Emotion and motivation: the role of the amygdala, ventral striatum, and prefrontal cortex. Neurosci Biobehav Rev 26: 321-52.

Carulli D, Rhodes KE, Brown DJ, Bonnert TP, Pollack SJ, Oliver K, et al (2006). Composition of perineuronal nets in the adult rat cerebellum and the cellular origin of their components. J Comp Neurol 494: 559-577. 
Chen BT, Yau HJ, Hatch C, Kusumoto-Yoshida I, Cho SL, Hopf FW, et al (2013). Rescuing cocaine-induced prefrontal cortex hypoactivity prevents compulsive cocaine seeking. Nature 496: 359-362.

Chen CH, Fremont R, Arteaga-Bracho EE, Khodakhah K (2014). Short latency cerebellar modulation of the basal ganglia. Nat Neurosci 17: 1767-1775.

Ciano P Di, Everitt BJ (2001). Dissociable effects of antagonism of NMDA and AMPA/KA receptors in the nucleus accumbens core and shell on cocaine-seeking behavior. Neuropsychopharmacology 25: 341-60.

Ciano P Di, Robbins TW, Everitt BJ (2008). Differential effects of nucleus accumbens core, shell, or dorsal striatal inactivations on the persistence, reacquisition, or reinstatement of responding for a drug-paired conditioned reinforcer. Neuropsychopharmacology 33: 1413-1425.

Cornish JL, Kalivas PW (2000). Glutamate transmission in the nucleus accumbens mediates relapse in cocaine addiction. J Neurosci 20: RC89.

Delatour B, Gisquet-Verrier P (2000). Functional role of rat prelimbic-infralimbic cortices in spatial memory: evidence for their involvement in attention and behavioural flexibility. Behav Brain Res 109: 113-28.

Desmond JE, Chen SHA, DeRosa E, Pryor MR, Pfefferbaum A, Sullivan E V (2003). Increased frontocerebellar activation in alcoholics during verbal working memory: An fMRI study. Neuroimage 19: 1510-1520.

Dias R, Aggleton JP (2000). Effects of selective excitotoxic prefrontal lesions on acquisition of nonmatching- and matching-to-place in the T-maze in the rat: Differential involvement of the prelimbic-infralimbic and anterior cingulate cortices in providing behavioural flexibility. Eur J Neurosci 12: 4457-4466.

Edward F (1992). Animal Models for Human. Prog Brain Res 85: 143-153.

Ettenberg A (1989). Dopamine, neuroleptics and reinforced behavior. Neurosci Biobehav Rev 13: 105-111.

Fallon JH, Moore RY (1978). Catecholamine innervation of the basal forebrain IV. Topography of the dopamine projection to the basal forebrain and neostriatum. $J$ Comp Neurol 180: 545-579.

Gil-Miravet I, Guarque-Chabrera J, Carbo-Gas M, Olucha-Bordonau F, Miquel M (2018). The role of the cerebellum in drug-cue associative memory: functional interactions with the medial prefrontal cortex. Eur J Neurosci 1-10doi:10.1111/ejn.14187.

Goldstein RZ, Volkow ND (2011). Dysfunction of the prefrontal cortex in addiction: Neuroimaging findings and clinical implications. Nat Rev Neurosci 12: 652-669.

Heidbreder CA, Groenewegen HJ (2003). The medial prefrontal cortex in the rat: Evidence for a dorso-ventral distinction based upon functional and anatomical characteristics. Neurosci Biobehav Rev 27: 555-579.

Hester R, Garavan H (2004). Executive Dysfunction in Cocaine Addiction: Evidence for Discordant Frontal, Cingulate, and Cerebellar Activity. J Neurosci 24: 11017-11022. 
Hoover WB, Vertes RP (2007). Anatomical analysis of afferent projections to the medial prefrontal cortex in the rat. Brain Struct Funct 212: 149-79.

Ikai Y, Takada M, Mizuno N (1994). Single neurons in the ventral tegmental area that project to both the cerebral and cerebellar cortical areas by way of axon collaterals. Neuroscience 61: 925-934.

Ikai Y, Takada M, Shinonaga Y, Mizuno N (1992). Dopaminergic and non-dopaminergic neurons in the ventral tegmental area of the rat project, respectively, to the cerebellar cortex and deep cerebellar nuclei. Neuroscience 51: 719-728.

Jentsch JD, Taylor JR (1999). Impulsivity resulting from frontostriatal dysfunction in drug abuse: implications for the control of behavior by reward-related stimuli. Psychopharmacology (Berl) 146: 373-90.

Koob GF, Swerdlow NR (1988). The Functional Output of the Mesolimbic Dopamine System. Ann N Y Acad Sci 537: 216-227.

LaLumiere RT, Niehoff KE, Kalivas PW (2010). The infralimbic cortex regulates the consolidation of extinction after cocaine self-administration. Learn Mem 17: 168175.

Lalumiere RT, Smith KC, Kalivas PW (2012). Neural circuit competition in cocaineseeking: Roles of the infralimbic cortex and nucleus accumbens shell. Eur J Neurosci 35: 614-622.

Lasseter HC, Xie X, Ramirez DR, Fuchs RA (2010). Prefrontal cortical regulation of drug seeking in animal models of drug relapse. Curr Top Behav Neurosci 3: 101-117.

Martín-García E, Courtin J, Renault P, Fiancette J-F, Wurtz H, Simonnet A, et al (2014). Frequency of cocaine self-administration influences drug seeking in the rat: optogenetic evidence for a role of the prelimbic cortex. Neuropsychopharmacology 39: 2317-30.

Martin-Sölch C, Magyar S, Künig G, Missimer J, Schultz W, Leenders K (2001). Changes in brain activation associated with reward processing in smokers and nonsmokers. Exp Brain Res 139: 278-286.

McFarland K, Davidge SB, Lapish CC, Kalivas PW (2004). Limbic and Motor Circuitry Underlying Footshock-Induced Reinstatement of Cocaine-Seeking Behavior. J Neurosci 24: 1551-1560.

McFarland K, Kalivas PW (2001). The circuitry mediating cocaine-induced reinstatement of drug-seeking behavior. J Neurosci 21: 8655-8663.

McFarland K, Lapish CC, Kalivas PW (2003). Prefrontal Glutamate Release into the Core ofthe Nucleus Accumbens Mediates Cocaine-Induced Reinstatement of DrugSeeking Behavior. J Neurosci 23: 3531-3537.

McLaughlin J, See RE (2003). Selective inactivation of the dorsomedial prefrontal cortex and the basolateral amygdala attenuates conditioned-cued reinstatement of extinguished cocaine-seeking behavior in rats. Psychopharmacology (Berl) 168: 5765 . 
Melchitzky DS, Lewis DA (2000). Tyrosine hydroxylase- and dopamine transporterimmunoreactive axons in the primate cerebellum: Evidence for a lobular- and laminar-specific dopamine innervation. Neuropsychopharmacology 22: 466-472.

Middleton FA, Strick PL (1994). Anatomical evidence for cerebellar and basal ganglia involvement in higher cognitive function. Science (80- ) 266: 458-461.

Middleton FA, Strick PL (2001). Cerebellar Projections to the Prefrontal Cortex of the Primate. J Neurosci 21: 700-712.

Milad MR, Quirk GJ (2002). Neurons in medial prefrontal cortex signal memory for fear extinction. Nature 420: 70-74.

Miquel M, Toledo R, Garcia L, Coria-Avila G, Manzo J (2009). Why Should We Keep the Cerebellum in Mind When Thinking About Addiction? Curr Drug Abus Rev 2: 26-40.

Miquel M, Vazquez-Sanroman D, Carbo-Gas M, Gil-Miravet I, Sanchis-Segura C, Carulli $D$, et al (2016). Have we been ignoring the elephant in the room? Seven arguments for considering the cerebellum as part of addiction circuitry. Neurosci Biobehav Rev 60: 1-11.

Moers-Hornikx VMP, Sesia T, Basar K, Lim LW, Hoogland G, Steinbusch HWM, et al (2009). Cerebellar nuclei are involved in impulsive behaviour. Behav Brain Res 203: 256-263.

Mogensen J, Holm S (1994). The prefrontal cortex and variants of sequential behaviour indications of functional differentiation between subdivisions of the rat's prefrontal cortex. Behav Brain Res 63: 89-100.

Moreno-Rius J, Miquel M (2017). The cerebellum in drug craving. Drug Alcohol Depend 173: $151-158$.

Morgan MA, Romanski LM, LeDoux JE (1993). Extinction of emotional learning: contribution of medial prefrontal cortex. Neurosci Lett 163: 109-13.

Moulton EA, Elman I, Becerra LR, Goldstein RZ, Borsook D (2014). The cerebellum and addiction: Insights gained from neuroimaging research. Addict Biol 19: 317-331.

Ongür D, Price JL (2000). The organization of networks within the orbital and medial prefrontal cortex of rats, monkeys and humans. Cereb cortex 10: 206-219.

Panagopoulos NT, Papadopoulos GC, Matsokis NA (1991). Dopaminergic innervation and binding in the rat cerebellum. Neurosci Lett 130: 208-212.

Paxinos G, Watson C (1998). The Rat Brain in Stereotaxic Coordinates. Acad Press 1474doi:10.1007/s13398-014-0173-7.2.

Peters J, Kalivas PW, Quirk GJ (2009). Extinction circuits for fear and addiction overlap in prefrontal cortex. Learn Mem 16: 279-288.

Peters J, LaLumiere RT, Kalivas PW (2008). Infralimbic prefrontal cortex is responsible for inhibiting cocaine seeking in extinguished rats. J Neurosci 28: 6046-53.

Pfarr S, Meinhardt MW, Klee ML, Hansson AC, Vengeliene V, Schonig K, et al (2015). Losing Control: Excessive Alcohol Seeking after Selective Inactivation of Cue- 
Responsive Neurons in the Infralimbic Cortex. J Neurosci 35: 10750-10761.

Ragozzino ME, Adams S, Kesner RP (1998). Differential involvement of the dorsal anterior cingulate and prelimbic-infralimbic areas of the rodent prefrontal cortex in spatial working memory. Behav Neurosci 112: 293-303.

Ragozzino ME, Detrick S, Kesner RP (1999). Involvement of the Prelimbic-Infralimbic Areas of the Rodent Prefrontal Cortex in Behavioral Flexibility for Place and Response Learning. J Neurosci 19: 4585-4594.

Rocha A, Kalivas PW (2010). Role of the prefrontal cortex and nucleus accumbens in reinstating methamphetamine seeking. Eur J Neurosci 31: 903-9.

Sang L, Qin W, Liu Y, Han W, Zhang Y, Jiang T, et al (2012). Resting-state functional connectivity of the vermal and hemispheric subregions of the cerebellum with both the cerebral cortical networks and subcortical structures. Neuroimage 61: 12131225.

Schindelin J, Arganda-Carreras I, Frise E, Kaynig V, Longair M, Pietzsch T, et al (2012). Fiji: an open-source platform for biological-image analysis. Nat Methods 9: 676-82.

Schmahmann JD, Pandya DN (1997). The cerebrocerebellar system. IntRevNeurobiol 41: 31-60.

Sesack SR, Deutch AY, Roth RH, Bunney BS (1989). Topographical organization of the efferent projections of the medial prefrontal cortex in the rat: An anterograde tract-tracing study with Phaseolus vulgaris leucoagglutinin. J Comp Neurol 290: 213-242.

Sierra-Mercado D, Padilla-Coreano N, Quirk GJ (2011). Dissociable roles of prelimbic and infralimbic cortices, ventral hippocampus, and basolateral amygdala in the expression and extinction of conditioned fear. Neuropsychopharmacology 36: 529538.

Slaker M, Churchill L, Todd RP, Blacktop JM, Zuloaga DG, Raber J, et al (2015). Removal of Perineuronal Nets in the Medial Prefrontal Cortex Impairs the Acquisition and Reconsolidation of a Cocaine-Induced Conditioned Place Preference Memory. J Neurosci 35: 4190-4202.

Stefanik MT, Kalivas PW (2013). Optogenetic dissection of basolateral amygdala projections during cue-induced reinstatement of cocaine seeking. Front Behav Neurosci 7: 213.

Vazquez-Sanroman D, Leto K, Cerezo-Garcia M, Carbo-Gas M, Sanchis-Segura C, Carulli $D$, et al (2015). The cerebellum on cocaine: Plasticity and metaplasticity. Addict Biol 20: 941-955.

Vertes RP (2004). Differential Projections of the Infralimbic and Prelimbic Cortex in the Rat. Synapse 51: 32-58.

Volkow ND, Wang G-J, Fowler JS, Tomasi D (2015). Addiction Circuitry in the Human Brain. Focus (Madison) 13: 341-350.

Voorn P, Vanderschuren LMJ, Groenewegen HJ, Robbins TW, Pennartz CMA (2004). 
Putting a spin on the dorsal-ventral divide of the striatum. Trends Neurosci 27: 468474.

Wang D, Fawcett J (2012). The perineuronal net and the control of CNS plasticity. Cell Tissue Res 349: 147-60.

Watson TC, Becker N, Apps R, Jones MW (2014). Back to front: cerebellar connections and interactions with the prefrontal cortex. Front Syst Neurosci 8: 4.

Xue Y-X, Xue L-F, Liu J-F, He J, Deng J-H, Sun S-C, et al (2014). Depletion of Perineuronal Nets in the Amygdala to Enhance the Erasure of Drug Memories. J Neurosci 34: 6647-6658.

Zavala AR, Weber SM, Rice HJ, Alleweireldt AT, Neisewander JL (2003). Role of the prelimbic subregion of the medial prefrontal cortex in acquisition, extinction, and reinstatement of cocaine-conditioned place preference. Brain Res 990: 157-64. 
EXPERIMENTAL STUDIES

Chapter 3 



\section{Chapter 3: From back to front: A functional model for the cerebellar modulation in the establishment of conditioned preferences for cocaine-related cues}

Isis Gil-Miravet ${ }^{1}$, Edgar Arias de Saavedra-Sandoval ${ }^{1}$, Lizbeth Vásquez-Celaya ${ }^{3}$, Julián Guarque-Chabrera $^{1}$, Francisco Olucha-Bordonau ${ }^{2}$, Marta Miquel ${ }^{1 *}$

1 Área de Psicobiología, Universitat Jaume I, Castellón de la Plana, Spain.

2 UP Medicina. Universitat Jaume I, Castellón de la Plana, Spain.

3 Centro de Investigaciones Cerebrales. Universidad Veracruzana, Xalapa, Mexico.

*Corresponding author. Área de Psicobiología, Universitat Jaume I, Avenida Vicente Sos Baynat sn, 12071, Castellón de la Plana, Spain.

miquel@uji.es

orcid.org/0000-0001-9670-4210 


\section{ABSTRACT}

It is now clear that the cerebellum may modulate brain functions altered in drug addiction. We previously demonstrated that cocaine-induced conditioned preference increased activity at the apical region of lobule VIII in the vermis. Activity in lobule VIII was significantly correlated with the level of preference towards cocaine-conditioned cues. Unexpectedly, a neurotoxic lesion of lobule VIII raised by up to one hundred the percentage of animals that acquired cocaine-induced conditioned preference. The present research aimed at providing an explanatory model for the facilitative effect of the cerebellar lesion on cocaine-induced conditioned memory. We evaluated cFos expression in different regions of the medial prefrontal cortex and striatum after a lesion in lobule VIII before conditioning. Additionally, to explore whether the cerebellar lesion might affect synaptic stabilization mechanisms in the medial prefrontal cortex, PNN expression was assessed. Damage in this region of the vermis induced a general disinhibition of the MPFC and striatal subdivisions that receive dopaminergic projections, mainly from the ventral tegmental area (VTA). Moreover, cerebellar impairment induced an upregulation of PNN expression in the mPFC. Finally, we addressed a tracing study using anterograde and retrograde tracers in order to build a working neuroanatomical model to explain the present results. We found a direct projection from the lateral nucleus to the VTA that also receives Purkinje axons from lobule VIII in the vermis. Hypothetically, this pathway might control activity and plasticity of the cortico-striatal circuitry through an increase in dopaminergic activity.

Keywords: cocaine, cerebellum, quinolinic acid, PNNs, VTA 


\section{INTRODUCTION}

For decades, the cerebellum's role has been restricted only to motor functions. Fortunately, in recent years, numerous investigations have described the involvement of the cerebellum in non-motor functions including language, spatial and emotional processing, reward, working memory, and executive functions (Ball et al, 1974; Carta et al, 2019; Corbett et al, 1982; Sacchetti et al, 2002; Schmahmann and Pandya, 1997; Turner et al, 2007; Wagner et al, 2017; Watson et al, 2014; Zhu et al, 2011). Anatomical and functional studies in rodents and non-human primates have shown extensive pathways that connect the cerebellum to the prefrontal cortex, striatum, amygdala, thalamus, hippocampus and basal ganglia (Bostan et al, 2018; Buckner et al, 2011; Chen et al, 2014; Hoshi et al, 2005; Ichinohe et al, 2000; Ikai et al, 1992; Middleton and Strick, 2000, 2001; Panagopoulos et al, 1991; Sang et al, 2012; Stanton, 1980; Xiao et al, 2018). More recently, two findings pointed to a direct control of the cerebellum over the ventral tegmental area (VTA) (Carta et al, 2019; Watabe-Uchida et al, 2012). All these results suggest that the cerebellum is part of cortical-striatal-limbic loops and may modulate brain functions altered in drug addiction (Miquel et al, 2009, 2016; Yalachkov et al, 2010).

Indeed, the cerebellum plays an important role in the consolidation of emotional memory, as well as in the establishment of automatic behavioural protocols (Callu et al, 2007; Sacchetti et al, 2002). Moreover, neuroimaging studies of drug-induced cue reactivity in drug addicts described cerebellar activation after the presentation of drugrelated cues (Anderson et al, 2006; Bonson et al, 2002; Fuentes et al, 2012; Grant et al, 1996; Moulton et al, 2014; Schneider et al, 2001). In a mice model of cocaine-induced conditioned preference, we showed that only those animals that developed preference for cocaine-related cues exhibited increased activity at the apical region of the cerebellar vermis (Carbo-Gas et al, 2014a, 2014b, 2017). Although this effect was found throughout the cerebellar cortex, only activity in lobule VIII was significantly correlated with the level of preference towards cocaine-related cues (Carbo-Gas et al, 2014b). Furthermore, cocaine-induced conditioned preference also increased the expression of perineuronal nets (PNNs) surrounding Golgi inhibitory interneurons located in the same region of the vermis (Carbo-Gas et al, 2017), suggesting that drug-induced Pavlovian memory 
encouraged one of the main mechanisms for synaptic stabilization (Sorg et al, 2016). On that basis, one could expect a neurotoxic lesion localised in lobule VIII to prevent the acquisition of cocaine-induced conditioned preference. On the contrary, the cerebellar lesion dramatically raised by up to 100 the percentage of rats that acquired cocaineinduced conditioned preference (Gil-Miravet et al, 2018). The same effect was observed after a reversible deactivation of the infralimbic (IL) cortex (Gil-Miravet et al, 2018). Moreover, simultaneous IL-cerebellar deactivation prevented the effect of either of the separate manipulations (Gil-Miravet et al, 2018). These results were in agreement with findings reporting that the IL cortex is required for the suppression of cocaine-seeking response and expression of extinction memory (Lalumiere et al, 2012; LaLumiere et al, 2010). Overall, our findings suggested that both the cerebellum and IL cortex might act together in regulating the establishment of drug-cue Pavlovian associations.

In the present work, we aimed at: (1) further investigating cerebellum-infralimbic functional relationships for the acquisition of cocaine-induced conditioned preference; and (2) proposing a functional model to explain the effects of the cerebellar lesion in cocaine-conditioned memory. We assessed cFos expression in different regions of the medial prefrontal cortex (MPFC) and striatum after a neurotoxic lesion in the apical region of lobule VIII before conditioning. Also, to explore whether the cerebellar lesion might affect synaptic stabilization mechanisms in the mPFC, PNN expression was evaluated. Finally, we addressed a tracing study using anterograde and retrograde tracers in order to build a working neuroanatomical model to explain the present results.

\section{METHODOLOGY}

\section{Subjects}

Twenty-two male Sprague-Dawley rats (Janvier, ST Berthevin Cedex, France) weighing between 175 and $200 \mathrm{~g}$ were randomly selected from the rats included in the first study (Gil-Miravet et al, 2018) (Chapter 1). Animals were individually housed under standard laboratory conditions, with controlled temperature and humidity (12 h light cycle from 8:00 a.m. to 8:00 p.m.) and access to food and water ad libitum (Jaume I University, Spain). Rats were handled and habituated to all of the experimental procedures. All 
animal procedures were approved by the local Animal Welfare Ethics Committee and Empowered Body (2014/VSC/PEA/00208) and developed in accordance with the European Community Council directive (2010/63/EU), Spanish directive BOE 34/11370/2013 and local directive DOGV 26/2010.

\section{Brain infusions and stereotactic surgery}

The animals were anaesthetized using a cocktail of ketamine $(100 \mathrm{mg} / \mathrm{Kg})$ (Imalgene 100 $\mathrm{mg} / \mathrm{ml}$, Mersal Laboratorios S.A., Barcelona, Spain) and xylazine $(10 \mathrm{mg} / \mathrm{kg}$ ) (Xylazine hydrochloride $\geq 99 \%$, Sigma-Aldrich, Madrid, Spain) (IP), and placed in a Kopf stereotaxic apparatus for the surgery. We use a stainless-steel guide cannula $(10 \mathrm{~mm}$ length; $23-$ gauge external diameter) for the intracranial infusion of quinolinic acid (QA) (90 $\mathrm{nmol} / \mu \mathrm{l}$ ) (2,3-Pyridinedicarboxylic acid, Sigma-Aldrich, Madrid, Spain) dissolved in phosphate buffered saline (PBS). The coordinates for the dorsal region of lobe VIII in the vermis were AP: -14.5; ML: 0; DV: -4.5 (Paxinos and Watson, 1998) (Fig. 1). QA infusion ( $0.5 \mu$ l volume; infusion ratio of $0.2 \mu \mathrm{l} / \mathrm{min}$ ) was released through a removable stainlesssteel injector (length, $11 \mathrm{~mm}$; external diameter, 30-gauge) inserted into the previously implanted guide cannula. After infusion, the cannula remained in place for 3 min to allow for diffusion. The same procedure was implemented in the sham group infusing PBS. After the surgery, all the animals received analgesic treatment with meloxicam (Metacam $5 \mathrm{mg} / \mathrm{ml}$, Boehringer Ingelheim, Barcelona, Spain) for 24 hours for three days. Cannula locations were verified by Nissl immunostaining and camera lucid. More detailed information can be found in (Gil-Miravet et al, 2018).

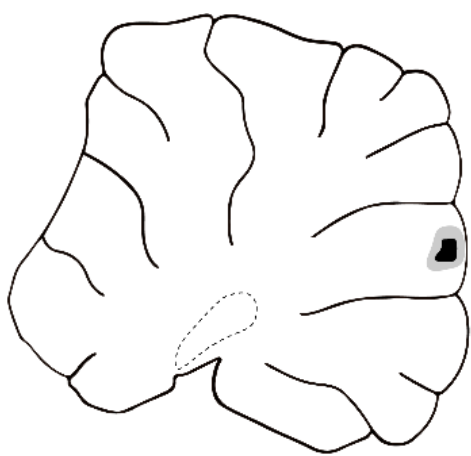

Lat: 0.90

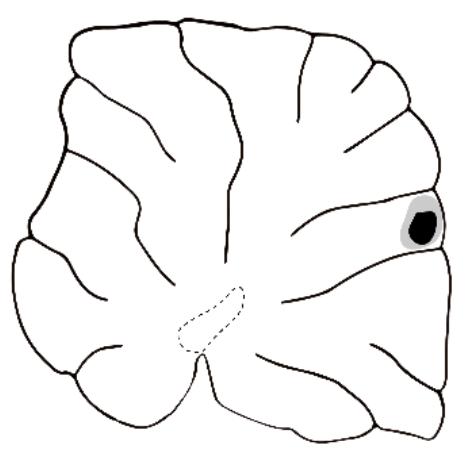

Lat: 0.40

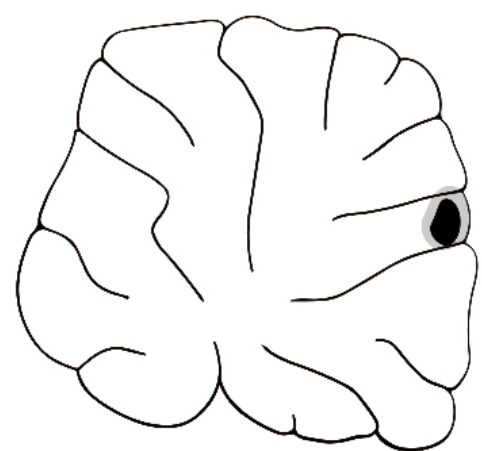

Lat: -0.10

Figure 1. Diagrams of the cannula locations. Schematic diagrams depicting the largest (grey) and smallest (black) diffusion areas in the apical region of lobule VIII in the vermis. The extent of the diffusion areas was assessed using light microscopy and camera lucida drawings. 
For the tracing study, retrograde and anterograde tracers were infused in different regions of the brain. As a retrograde tracer, we used FluoroGold with DAPI (FG) (Hydroxystilbamidine, Biotium, Barcelona, Spain). The anterograde tracing was accomplished using Dextran Biotin (BDA) (10,000 MW, Lysine Fixable, Thermo Fisher Scientific, Barcelona, Spain). The following coordinates were used: IL (AP: +3.2; ML: + 0.6/-0.6; DV: -5); VTA (AP: -5.2; ML: + 0.9/-0.9; DV: -8.3); lateral nucleus (Lat) (AP: 11.4; ML: + 3.6/-3.6; DV: -6.2); interpositus nucleus, anterior part (IntA) (AP: -11.3; ML:

+ 2.5/-2.5; DV: -5.8); interpositus nucleus, posterior part (IntP) (AP: -11.7; ML: + 2.5/-2.5; DV: -6.2); medial nucleus (Med) (AP: -11.4; ML: + 1/-1; DV: -6.2); and the apical area of lobe VIII in the vermis (AP: -14.5; ML: 0; DV: -4.5) (Paxinos and Watson, 1998). FG or BDA infusion volumes were $0.5 \mu \mathrm{l}$ in the IL and $0.3 \mu \mathrm{l}$ in the rest of regions with an infusion ratio of $0.2 \mu \mathrm{l} / \mathrm{min}$. After infusions, the rats remained undisturbed for ten days before perfusion.

\section{Cocaine-induced preference conditioning procedure}

The cocaine-induced conditioning procedure has been published previously (Gil-Miravet et al, 2018). Briefly, conditioning was conducted using two equally preferred olfactory stimuli located in the walls of a black chamber $(20 \times 20 \times 60 \mathrm{~cm})$ at the opposite arms of a corridor. One of the odours acted as the conditioned stimulus (CS+) and was associated with an IP injection of cocaine hydrochloride $(15 \mathrm{mg} / \mathrm{kg}$, IP) (Alcaliber S.A., Madrid, Spain). On alternate days, rats were exposed to the other scent (CS-) placed at the opposite black chamber in the corridor and received $0.9 \%$ saline injections. During pairing sessions (15 $\mathrm{min}$ ) animals remained confined in the chamber. A total of eight cocaine-paired sessions were conducted. The olfactory cues and locations in the corridor were counterbalanced between animals. Preference for the cocaine-related cue was evaluated $48 \mathrm{~h}$ after the last cocaine administration in a 30 min drug-free test in which CS+ and CS- odours were present simultaneously but in opposite arms of the corridor. The first ten minutes were not considered in order to allow the animal to explore the location of the odours, which was the opposite to the conditioning phase. The preference score was calculated as [TS in CS+ / (TS in CS+ + TS in CS-)] × 100. Additionally, we included a pseudo-conditioning group (the Unp group) that was treated with the same number of cocaine injections, but randomly associated with the olfactory stimuli. 
The Unp group allowed us to test for memory-related effects of our cerebellar deactivations. Animals were perfused transcardially 90 min following the preference test.

\section{Immunohistochemistry and immunofluorescence}

Animals were deeply anaesthetised with sodium pentobarbital $(30 \mathrm{mg} / \mathrm{kg}$ ) (Dolethal 100 ml, Vetoquinol E.V.S.A., Madrid, Spain) and perfused transcardially with saline (0.9\%) and paraformaldehyde (4\%) 90 min following the preference test. After perfusion, the brain and cerebellum were quickly dissected and placed in a container with the same fixative for $24 \mathrm{~h}$ at $4 \stackrel{\circ}{ } \mathrm{C}$. Then, the tissue was immersed in sucrose solution (30\%). The brain tissue was frozen with liquid nitrogen, and sections were performed at $40 \mu \mathrm{m}$ with a cryostat microtome (Microm HM560, Thermo Fisher Scientific, Barcelona, Spain). Eight series of tissue sections were collected and stored at $-80^{\circ} \mathrm{C}$ in cryoprotectant solution. Lesion sites were localized and represented using light microscopy and camera lucida drawings. Animals with cannula misplacement were not included in the statistical analysis.

Immunolabelling was performed on free-floating sections. For cFos peroxidative immunostaining, tissue peroxidases were eliminated and the brain tissue was incubated for $48 \mathrm{~h}$ with a polyclonal primary antibody, rabbit anti-cFos (1:1000; Synaptic Systems, Goettingen, Germany) and then, for $120 \mathrm{~min}$ with an affinity purified secondary biotinylated antibody, goat anti-rabbit (1:400; Jackson InmunoResearch Laboratories, Inc., West Grove, PA, USA). For magnification, we used preassembled biotin-avidin peroxidase complex according to the Vector Labs recommendations ( $A B C$ Elite; Vector Laboratories, Bulingame, Ca, USA). Sections were exposed to DAB solution with nickel. Then the tissue was rinsed and mounted in Eukitt (Sigma-Aldrich, Madrid, Spain).

For the double fluorescence immunolabelling of PNNs and cFos, brain tissue was incubated with lectin from Wisteria Floribunda Agglutinin (WFA) (1:200; Sigma-Aldrich, Madrid, Spain) and the polyclonal primary antibody, rabbit anti-cFos (1:1000; Synaptic Systems, Goettingen, Germany) at $4{ }^{\circ} \mathrm{C}$ for 48 hours in PBS $0.1 \mathrm{M}$ triton X-100. In a second step, brain samples were exposed to FICT-Streptavidin (1:50; Jackson InmunoResearch 
Laboratories, Inc., West Grove, PA, USA) and goat anti-rabbit Cy5 (1:200, Synaptic systems, Goettingen, Germany).

The anterograde tracer BDA was revealed using Cy3-conjugated Streptavidin (1:300; Jackson InmunoResearch Laboratories, Inc., West Grove, PA, USA). Different transporters were marked at the same time as the tracers. In a first step, brain tissue was incubated with rabbit anti-Calbindin 28k (1:1500; Swant, Marly, Switzerland) and guinea pig Anti-VGAT cytoplasmic (1:100; Synaptic Systems, Goettingen, Germany), and then exposed to donkey anti-rabbit Alexa 488 (1:500; Thermo Fisher Scientific, Geel, Belgium) and goat anti-guinea pig Alexa 647 (1:500; Thermo Fisher Scientific, Geel, Belgium). Different sections were incubated with rabbit anti-Tyrosine Hydroxylase (1:500; Millipore Merck KGaA, Darmstadt, Germany) and mouse anti-Synapsin1 (1:500; Synaptic Systems, Goettingen, Germany), and then exposed to goat anti-rabbit FITC (1:200; Vector Laboratories, Bulingame, Ca, USA) and donkey anti-mouse Alexa 647 (1:500; Jackson InmunoResearch Laboratories, Inc., West Grove, PA, USA). In the last fluorescence immunostaining, tissue was incubated with rabbit anti-Tyrosine Hydroxylase (1:500; Millipore Merck KGaA, Darmstadt, Germany) and guinea pig antivGluT2 (1:500; Synaptic Systems, Goettingen, Germany), and then exposed to goat antirabbit FITC (1:200; Vector Laboratories, Bulingame, Ca, USA) and goat anti-guinea pig Alexa 647 (1:500; Thermo Fisher Scientific, Geel, Belgium). All the sections were mounted with Mowiol.

\section{Image acquisition and analysis}

Images of immunoperoxidase cFos expression were acquired using an optic microscope (Nikon E-800, Izasa Werfen Group, Valencia, Spain) with 20x lenses and a resolution of 1,360 x 1,024 dpi. Three photos were taken by structure (IL, PL, dorsomedial striatum (DMS), dorsolateral striatum (DLS), ventrolateral striatum (VLS), nucleus accumbens core (NAcC) and shell (NAcSh)), and hemisphere in coronal sections. We included bregma coordinates between $3.20 \mathrm{~mm}$ to $2.20 \mathrm{~mm}$ for PL and IL, and for the striatum and NAc between $1.60 \mathrm{~mm}$ to $0.70 \mathrm{~mm}$.

Fluorescence images of cFos and PNNs were captured in a confocal microscope (Leica DMi8, Leica Microsystems CMS GmbH, Wetzlar, Germany) with 20x lenses and 
resolution $1,024 \times 1,024 \mathrm{dpi}$. Laser intensity (1\%), gain (600) and offset (-4) were maintained constant in each acquisition. Each image was formed by a stack of ten images. Image stacks were pre-processed applying a maximal projection process with Leica Application Suite LAS X (Leica Microsystems CMS GmbH, Wetzlar, Germany). Three image stacks in coronal sections for brain regions and in sagittal sections for deep cerebellar nuclei were acquired by structure and hemisphere.

FIJI free software (Schindelin et al, 2012) was used for all quantifications. The cFos expression was evaluated using the cell-counter plugin of FIJI software. Additionally, PNN expression was estimated using a densitometry assessment of WFA intensity (brightness range 0-255) in all the PNNs that were found in three sections of each ROI (Carbo-Gas et al, 2017; Vazquez-Sanroman et al, 2015a, 2015b). Unmodified images were used for all the analyses.

Fluorescent images of the infusion locations, tracer diffusion and transporters were acquired as eight confocal stacked images using the tile-scan tool in order to obtain complete coronal sections in which ROls were presented.

\section{Experimental design and statistics}

All statistical analyses were performed using GraphPad Prism 7 software (GraphPad Software Inc., La Jolla, CA, USA). One-way ANOVAs and Student t-tests for independent samples were carried out to analyse preference scores and immunohistochemistry data. Data were presented as mean \pm SEM. Post hoc comparisons were performed using Tukey's HSD tests. The statistical level of significance was set at $\mathrm{P}<0.05$.

\section{RESULTS}

In chapter 1 (Gil-Miravet et al, 2018), we showed that an excitotoxic lesion in the apical region (dorsal) of lobule VIII before conditioning facilitated cocaine-induced conditioned preference. Indeed, the whole sample of lesioned animals developed a preference for the cocaine-related cue, as compared to only one third of the sham group. In the present study, we confirmed the results with the smaller sample of rats $(N=18)$ in which immunohistochemistry studies were conducted (the sham group $(n=6)$, the lesioned 
group (QA) $(n=6)$, and the pseudo-conditioned group (Unp) $(n=6))$. As expected, the cerebellar lesion promoted the acquisition of cocaine-induced conditioned preference $(F(2,15)=5.337, P=0.0178)$. Post hoc analysis revealed that the QA group showed significant higher preference that the sham $(P=0.0463)$ and Unp $(P=0.0235)$ groups. No difference was found between the sham and Unp groups $(P=0.9350)$. Remarkably, the neurotoxic lesion was ineffective in affecting the behaviour of the Unp group, indicating that any effect of the lesion was learning-related (Fig. 2).

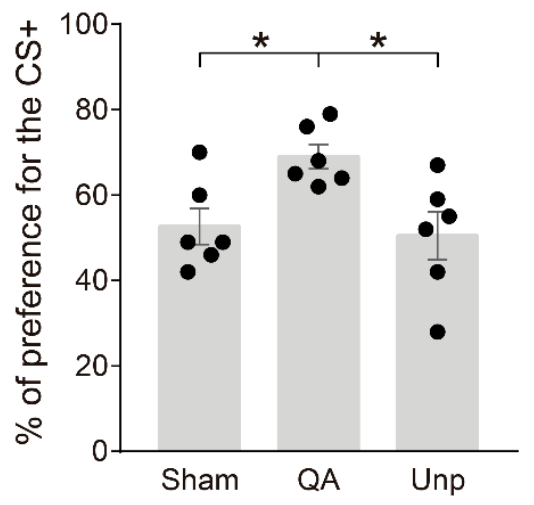

Figure 2. Effect of $A Q$ lesion before each training session on cocaine-induced conditioned preference. The QA lesion increased the proportion of rats that expressed cocaine-induced conditioned preference. Scatterplots of the preference scores for the CS+ on the test day in the sham $(n=6)$, QA lesion $(Q A)(n=6)$ and unpaired (Unp) $(n=6)$ groups. Data are shown as the mean \pm SEM and individual preference scores $\left({ }^{*} p<0.05\right)$.

\section{A lesion of lobule VIII in the vermis increases neural activity and PNN expression in the medial prefrontal cortex}

Then, we explored the impact of the cerebellar lesion on neural activity (cFos) and PNN expression (WFA) in the medial prefrontal cortex of these rats. As shown in figure 3 , the cerebellar lesion increased cFos expression in both the PL $(F(2,15)=13.4, P=0.0005)$ and the $\operatorname{IL}(F(2,15)=12.23, P=0.0007)$ subdivisions of the mPFC. In the IL cortex, Tukey tests showed higher number of cFos+ neurons in the lesion group as compared to the sham $(P=0.004)$ and unpaired $(P=0.0009)$ groups. The number of cFos+ neurons also increased significantly in the PL region of the lesioned rats as compared to the sham ( $P$ $=0.0018)$ and unpaired $(P=0.0008)$ groups.

Moreover, the lesion in lobule VIII enhanced WFA intensity in PNNs around GABAergic interneurons either in the $\operatorname{PL}(F(2,15)=7.262, P=0.0062)$ or $\operatorname{IL}(F(2,15)=18.03, P=$ 0.0001 ) cortex. Post hoc tests indicated that prefrontal PNNs were stronger after the 

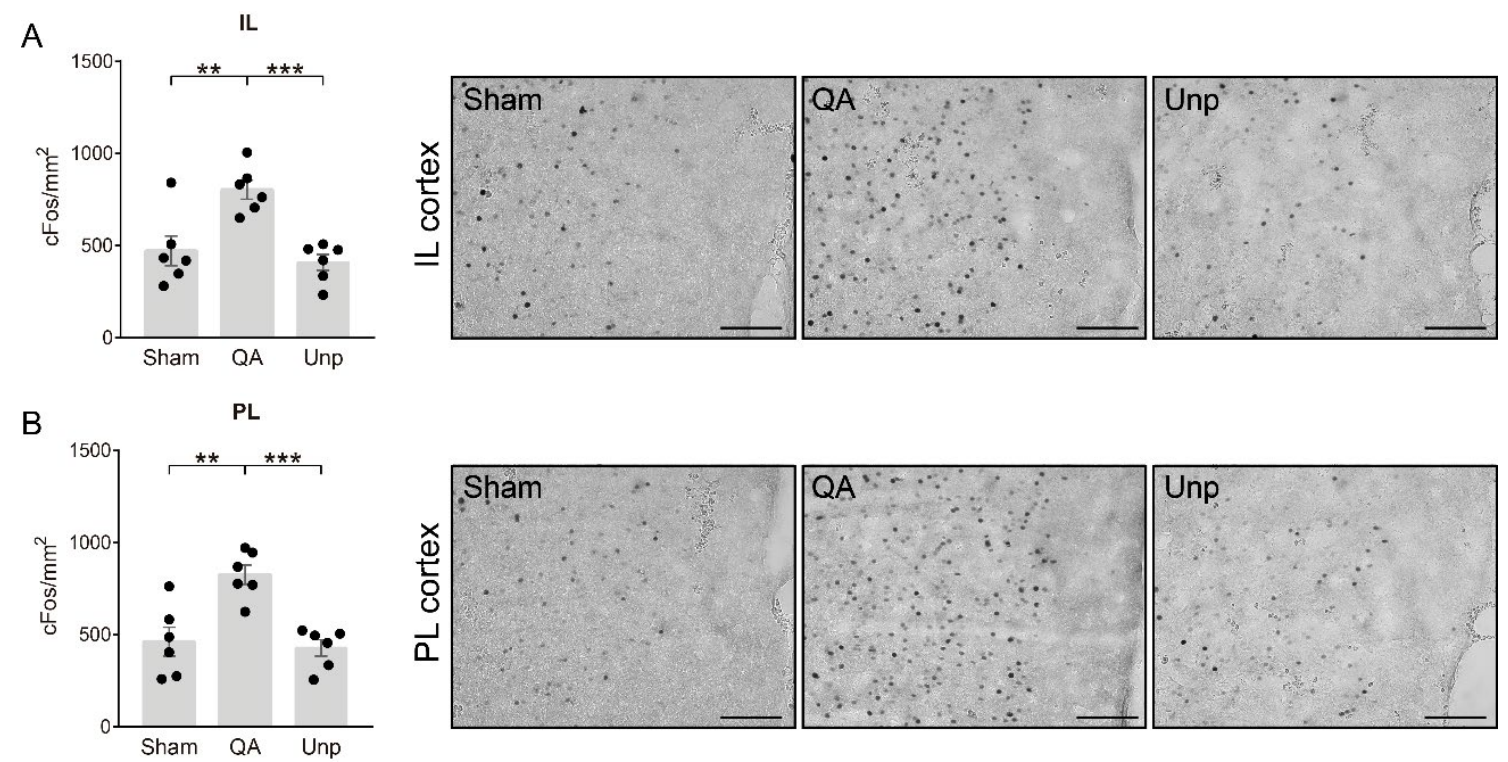

Figure 3. Effect of an excitotoxic lesion in lobule VIII of the vermis on cFos activity in the mPFC. The cerebellar lesion was made before cocaine-induced conditioning training. cFos expression in PL and IL cortices for Sham $(n=6), Q A(n=6)$ and Unp $(n=6)$ groups. (A) the PL and $(B)$ IL cortices for Sham $(n=6)$, $Q A(n=6)$ and Unp $(n=6)$ groups. The cerebellar lesion increased cFos expression in both subdivisions of the MPFC. The lesion was ineffective in producing both effects when cocaine was randomly associated with the odour cues (Unp). Data are shown as the mean \pm SEM and individual scores $\left({ }^{* *} P<0.01 ;{ }^{* * *} P<\right.$ $0.001)$. All images on the right panels were taken at 20x magnification. Scale bar $100 \mu \mathrm{m}$.

A

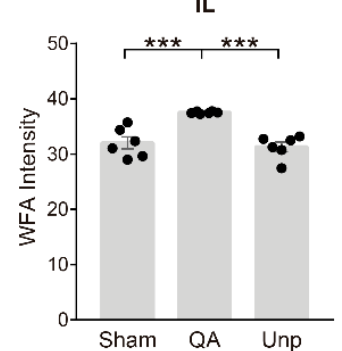

B

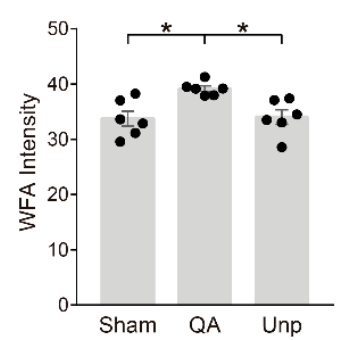

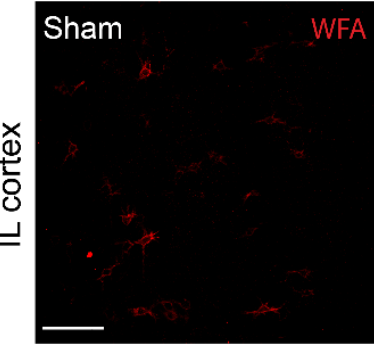

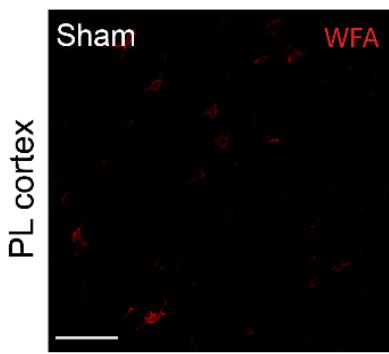

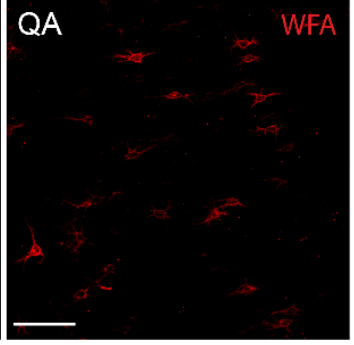
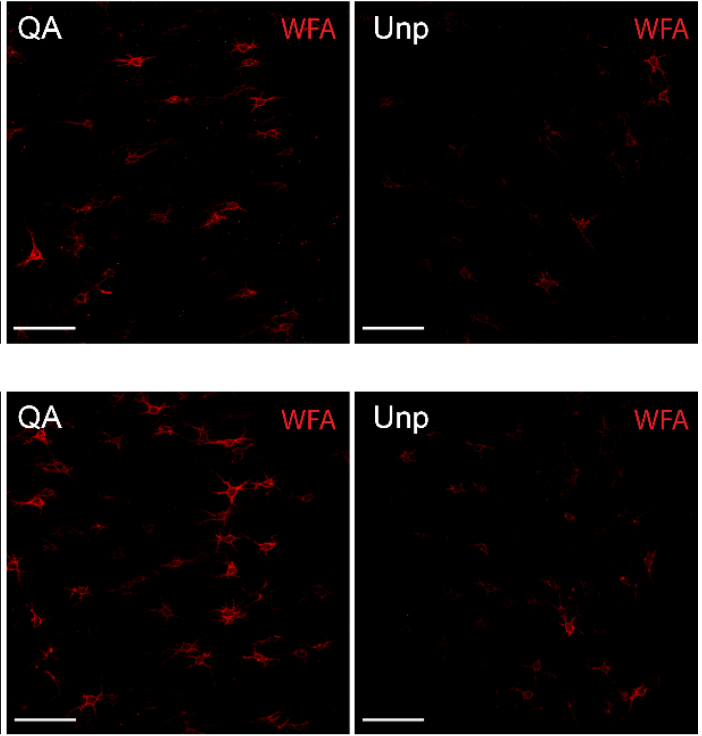

Figure 4. Effect of an excitotoxic lesion in lobule VIII of the vermis on PNNs expression in the mPFC. WFA intensity in PNNs in the PL and IL cortices for the Sham, QA and Unp groups ( $n=6)$. The cerebellar lesion increased WFA intensity in both subdivisions of the MPFC. The lesion was ineffective in producing both effects when cocaine was randomly associated with the odor cues. Data are shown as the mean \pm SEM and individual scores ( $\left.{ }^{*} P<0.05 ; * * *<0.001\right)$. Right, representative microphotographs of PNNs in the IL and PL cortex stained with Wisteria Floribunda Agglutinin (WFA) (red). The confocal images were acquired at 20x. Scale bar of $100 \mu \mathrm{m}$. 
cerebellar lesion as compared to the sham (PL $(P=0.0108) ; \mathrm{IL}(P=0.0006))$ and Unp (PL $(P=0.0151) ; \mathrm{IL}(P=0.0002))$ groups (Fig. 4).

\section{Neural activity in the NAC and striatum increased after the lesion of lobule VIII}

Additionally, we wonder whether cerebellar impairment during the acquisition of cocaine-induced conditioned memory might affect striatal activity. Student's t-test for independent samples showed that the cerebellar lesion raised cFos expression in the NAc and the majority of subdivisions of the striatum as compared to the sham group (NAcC (t $(10)=3.479, P=0.0059) ; \operatorname{NAcSh}(t)(10)=5.062, \mathrm{P}=0.0005) ; \mathrm{DMS}(\mathrm{t}(10)=2.676$, $\mathrm{P}=0.0233)$; and $\mathrm{DLS}(\mathrm{t}(10)=4.761, \mathrm{P}=0.0008))$. The only striatal region unaffected by the cerebellar lesion was the VLS $(T(10)=1.526, P=0.158)$ (Fig. 5).
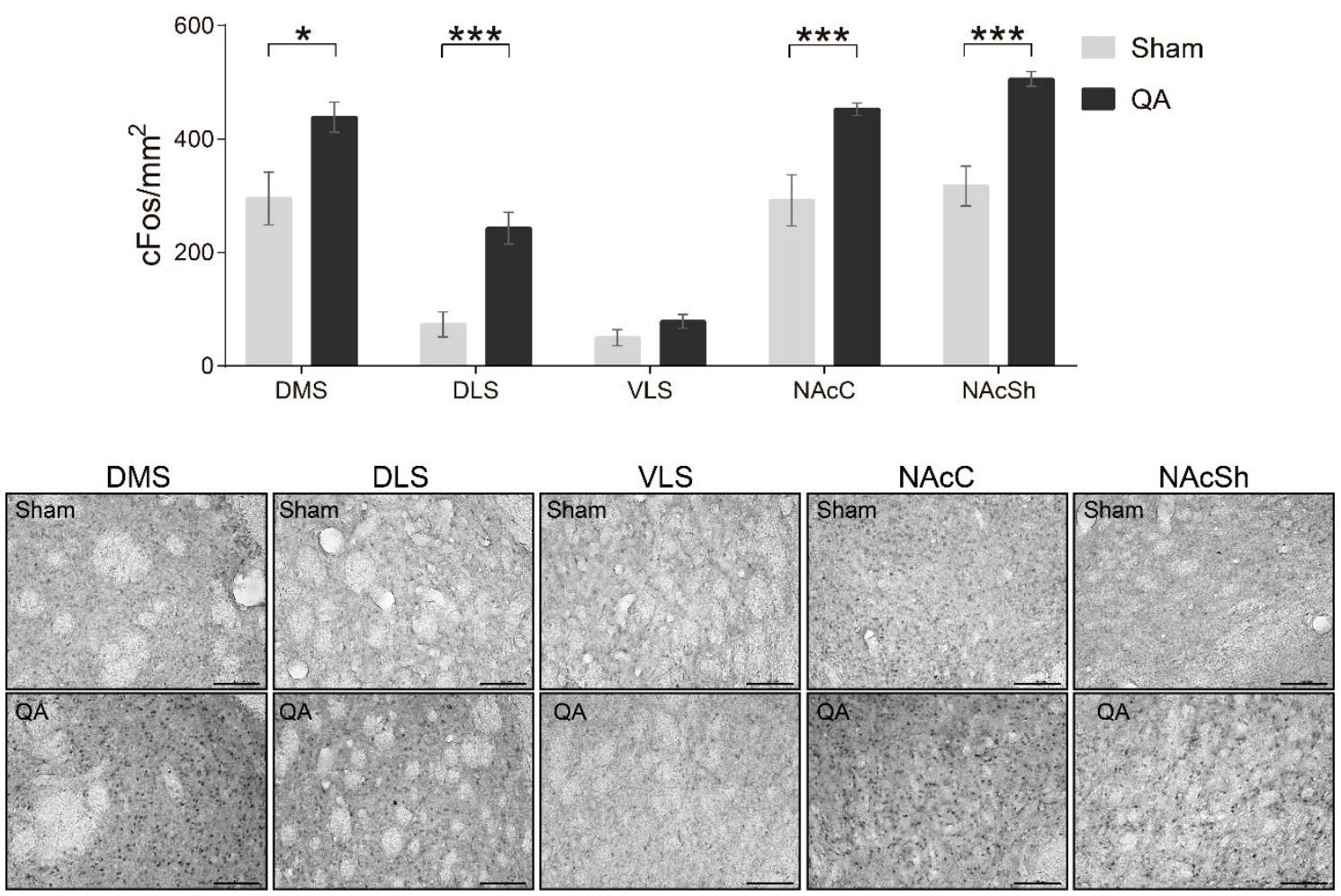

Figure 5. Effect of an excitotoxic lesion in lobule VIII on cFos expression in several striatal regions and the nucleus accumbens. DMS, DLS, VLS, NACC and NACSh in the sham and QA groups (n=6). Data are shown as the mean \pm SEM. The cerebellar lesion increased cFos expression in DMS, DLS, NAcC and NAcSh but not in the $V L S\left({ }^{*} p<0.05 ;{ }^{* *} p<0.001\right)$. All images were taken at $20 x$ magnification. Scale bar is equal to $100 \mu \mathrm{m}$. 


\section{A lesion in lobule VIII increases neural activity and PNNs expression in the lateral nucleus}

Student's t-tests revealed that the lesion of the vermis did not significantly change the cFos expression in the interpositus nucleus, either anterior $(\operatorname{Int} A)(t(10)=1.48, P=$ $0.1697)$ or posterior (IntP) $(T(10)=2.037, P=0.069)$ (Fig. 6). Neither PNNs in the IntA ( $P$ $=0.3384)$ nor in the IntP $(P=0.5832)$ were affected by the lesion (see also Fig. 7).

However, it is remarkable that the lesion in the posterior vermis increased cFos $(t)(10)=$ 2.597, $P=0.0266$ ) in the lateral nucleus (Lat) (Fig. 6). Moreover, WFA intensity was stronger in PNNs of this nucleus after the lesion of the posterior vermis $(\mathrm{t}(10)=2.614$, $P=0.0259)$ (Fig. 7).
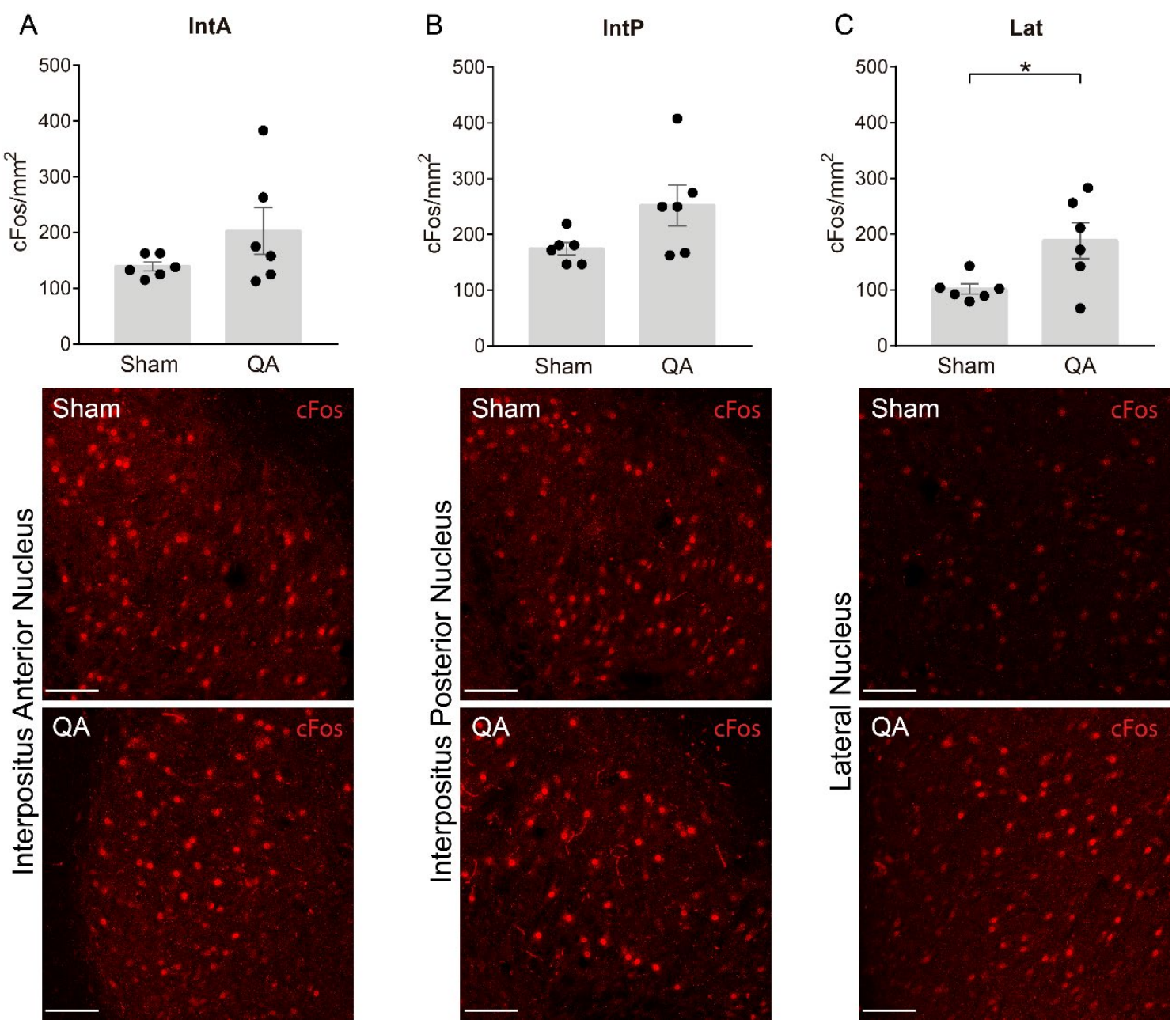

Figure 6. Effect of a lesion of lobule VIII in the vermis on cFos activity in the DCN. cFos expression rose in the Lat nucleus as an effect of the lesion of the posterior vermis $(n=6)$. Bottom panels depict representative pictures of cFos staining (red) for each group. All images were taken at 20x magnification. Scale bar 100 $\mu m$. Data are shown as the mean $\pm S E M$. Scatterplots were overlapped for each group $\left({ }^{*} P<0.05\right)$. 

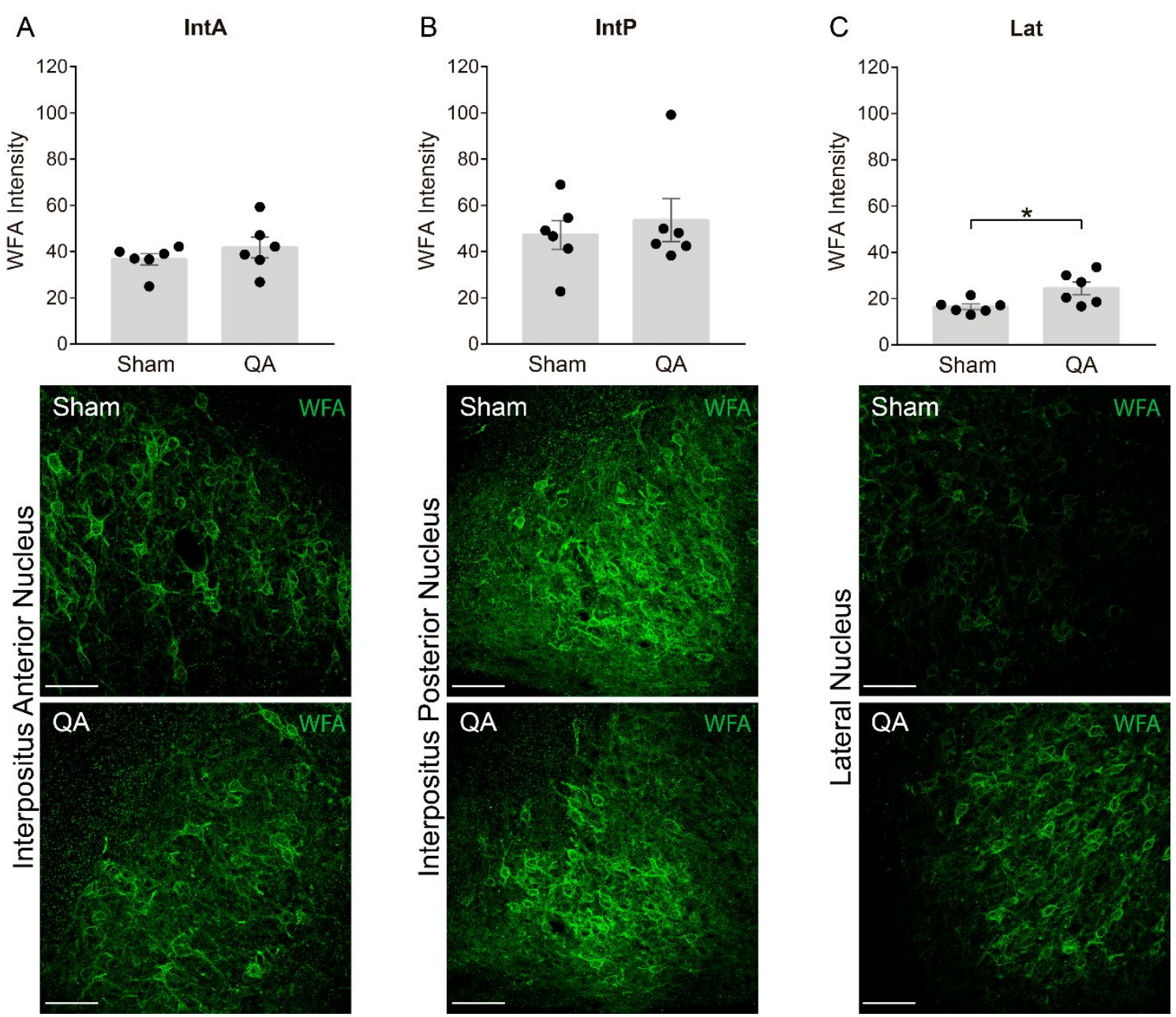

Figure 7. Effect of a lesion of lobule VIII in the vermis on WFA intensity in the lateral nucleus. PNN expression increased in the Lat nucleus as an effect of the lesion in the posterior vermis $(n=6)$. Bottom: representative microphotographs of PNNs stained with Wisteria Floribunda Agglutinin (WFA) (green). The confocal images were acquired at 20x. Scale bar $100 \mu \mathrm{m}$. Data are shown as the mean \pm SEM. Scatterplots were overlapped for each group $(* P<0.05)$.

\section{A working neuroanatomical model for the cerebellar regulation of cocaine-induced conditioned preference}

To propose a working neuroanatomical model to explain the effects of the lesion in the posterior vermis, we carried out a tracing study using anterograde (BDA/red) and retrograde (FG/blue) tracers. BDA was infused into the apical region of lobule VIII in the vermis (the same location as the excitotoxic lesion) and the lateral nucleus. In turn, we infused FG into the VTA and IL cortex. We searched for colocalization between BDA and FG within the VTA and lateral nucleus ( $n=3$ ) (Figs 8-9).

Despite the fact that the infusion point was restricted to lobule VIII in the vermis (Fig. 8), BDA-labelled projections were found throughout the whole vermis, but also the hemispheres reaching Crus I. However, the molecular layer was devoid of BDA labelling. 

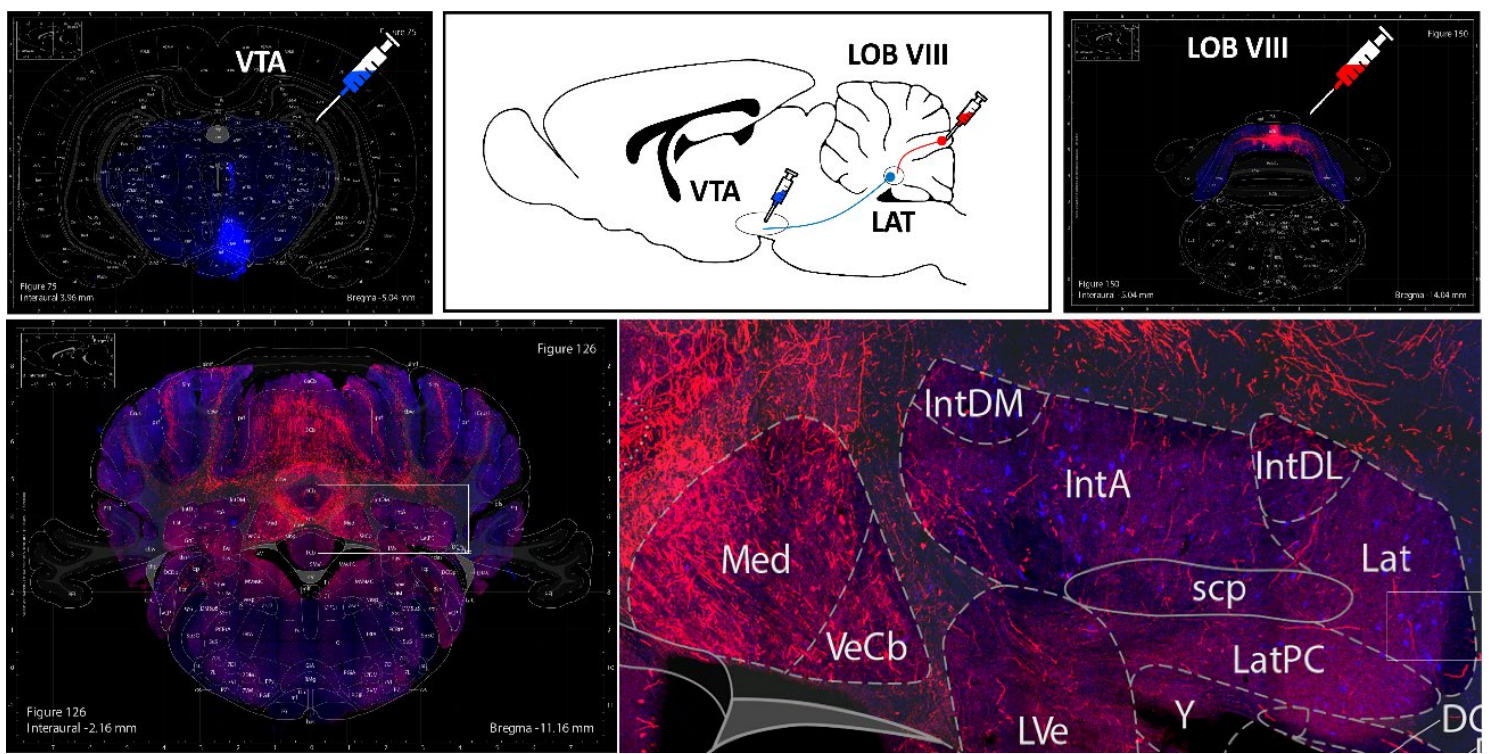

Figure 8. Infusions of FG (blue) in the VTA and BDA (red) in the dorsal region of lobule VIII in the vermis. Upper left, the infusion point for FG in the VTA. Upper right, the infusion point for BDA in lobule VIII of the vermis. BDA diffusion was found not only in the vermis but also throughout the hemispheres. Colocalization was only found within the IntA and Lat nuclei.

BDA is an anterograde tracer that identifies only a neural projection from their source to their point of termination. Hence, one plausible explanation for these results is that Purkinje-to-Purkinje collaterals would have spread the tracer laterally from the middle line along the cerebellar cortex. Purkinje collateral branches originate within the parasagittal plane and their maximum length can reach $2 \mathrm{~mm}$ towards the apex and the base of the lobule in adult rodents (Witter et al, 2016). Importantly, these findings suggest that lobule VIII is interconnected with the whole cerebellum. As expected, we
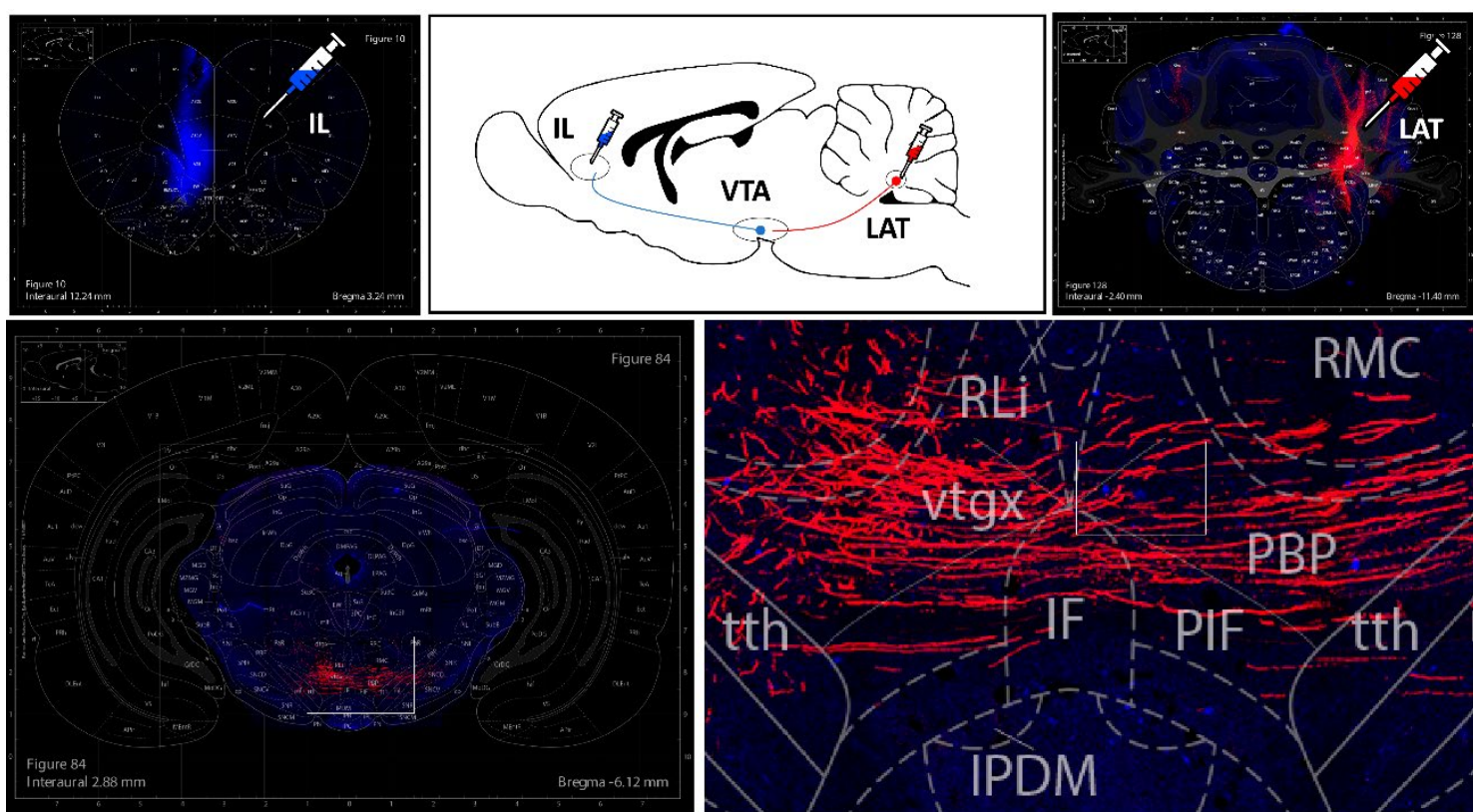

Figure 9. Infusions of FG (blue) in the IL cortex and BDA (red) in the lateral nucleus. Colocalization was observed within the contralateral parabrachial pigmented nucleus of the VTA (PBP). 


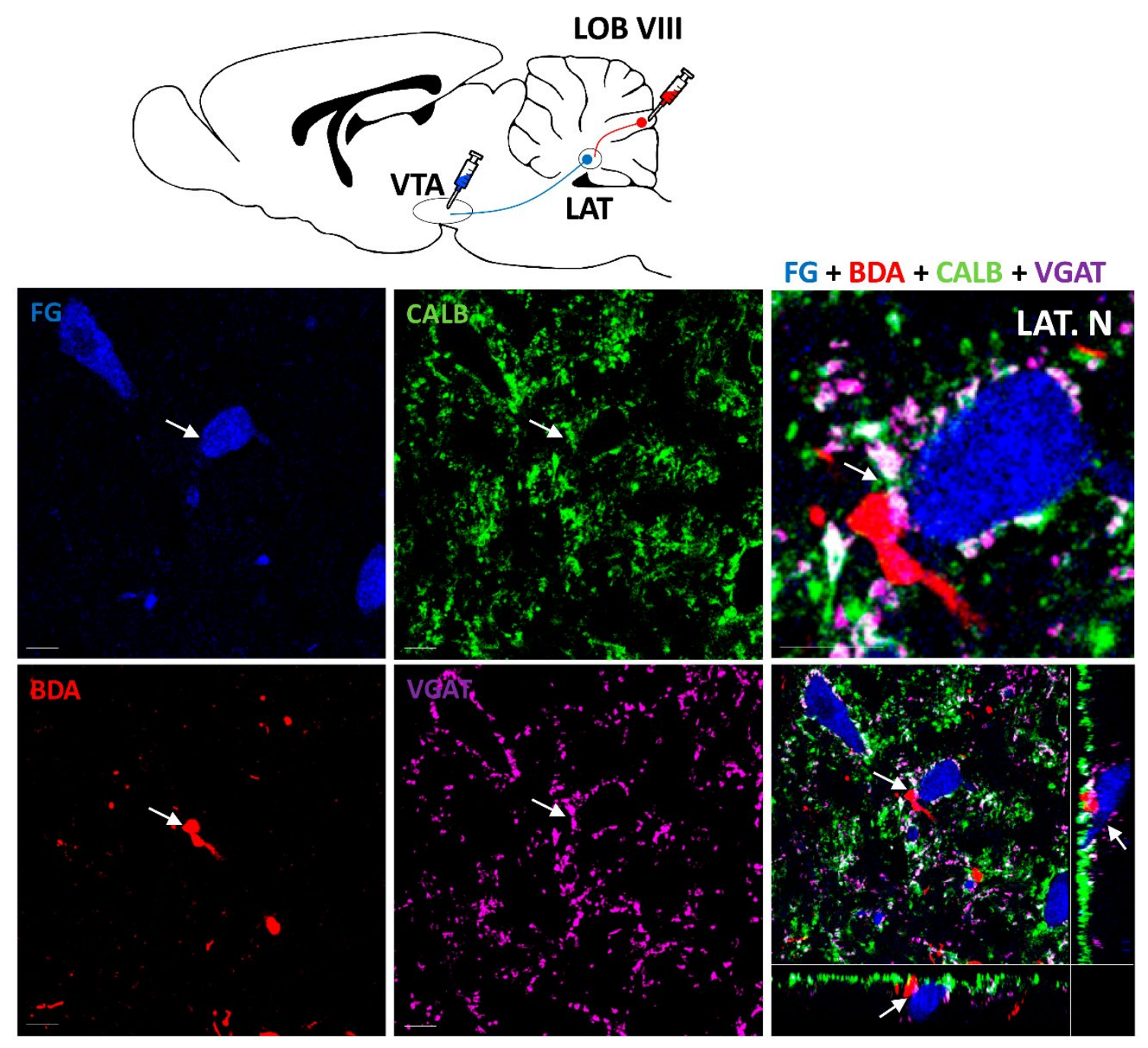

Figure 10. A coronal section of the lateral nucleus. Infusion of BDA (red) into the apical region of the vermis and FG (blue) in the VTA. The arrows indicate an example of a synaptic contact between a Purkinje terminal from lobule VIII of the vermis (BDA+) and one output neuron in the Lat (FG+). Purkinje puncta was identified by calbindin (CALB/green) and the GABA vesicular transporter (vGAT/purple). All confocal images were taken at 40x with 2.5x zoom. Scale bar $10 \mu \mathrm{m}$. Right top panel: Digital amplification of 300x.

observed a great number of BDA-labelled terminals within the medial nucleus, but also in the interpositus (Int) and lateral (Lat) nuclei (Fig. 8).

When FG was infused into the VTA, FG-labelled somas were found within the contralateral IntA, IntP, and Lat nuclei, but not in the Med nucleus (Fig. 8). This cerebellum-VTA projection was confirmed by infusing BDA into the Lat nucleus (Fig. 9). Unilateral BDA infusions in the Lat nucleus reached ipsilateral cerebellar hemisphere, lobule VIII and IX of the vermis, and contralateral parabrachial pigmented nucleus of the VTA (PBP), the most caudal part of the VTA (Fig. 9). We also observed FG+ somas in mPFC and NAcSh, among other structures, replicating the results observed in different studies (Geisler and Zahm, 2005; Kasanetz et al, 2008). A magnification image within the Lat 


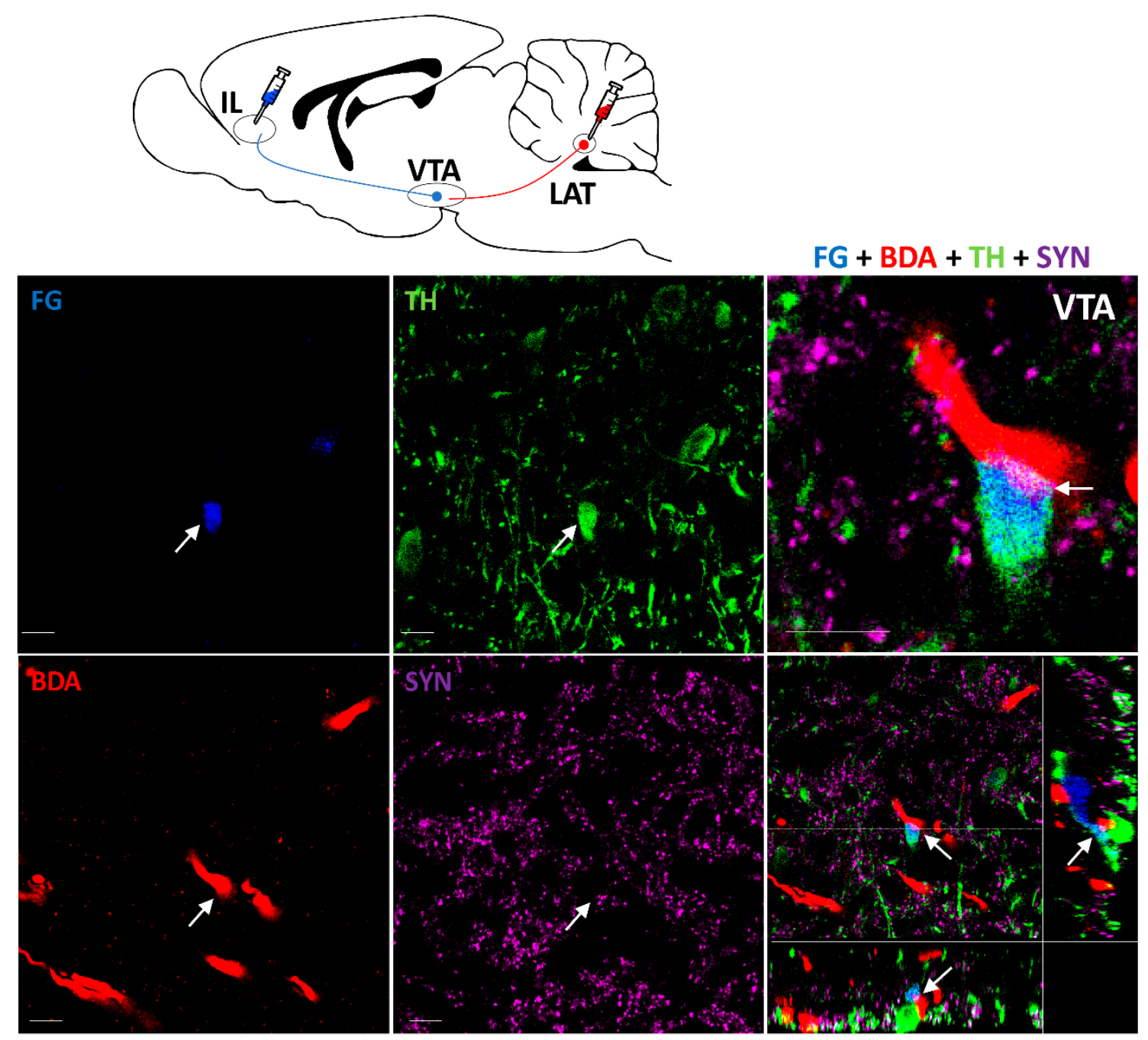

Figure 11. Coronal section of the VTA. Infusions of BDA (red) in the lateral nucleus and FG (blue) in the IL cortex. The arrows indicate an example of a synaptic contact between the terminal of a projection neuron from Lat nucleus (BDA+) and one $\mathrm{TH}+$ neuron in the VTA (FG+). Dopamine neuron was identified as expressing tyrosine hydroxylase (TH/green). Synapsin is a presynaptic protein (SYN/purple). All the pictures are taken a $40 x$ and $2.5 x$ zoom in a confocal. Scale bar of $10 \mu \mathrm{m}$. Digital co-labelling amplification of 300x. nucleus showed an example of a synaptic contact between a Purkinje BDA+ terminal from lobule VIII and FG+ cerebellar-VTA projection (Fig 10).

Additionally, FG was infused in the IL cortex and BDA in the Lat $(n=2)$. We observed FG and BDA co-labelling within the PBP (Fig. 9), supporting the mentioned observations and pointing to the caudal VTA as an interface through which the cerebellum would regulate prefrontal activity and striatal function. Projections from the lateral nucleus seemed to be glutamatergic and contacted both $\mathrm{TH}+$ and $\mathrm{TH}$ - cells (Fig. 11, 12, 13). 


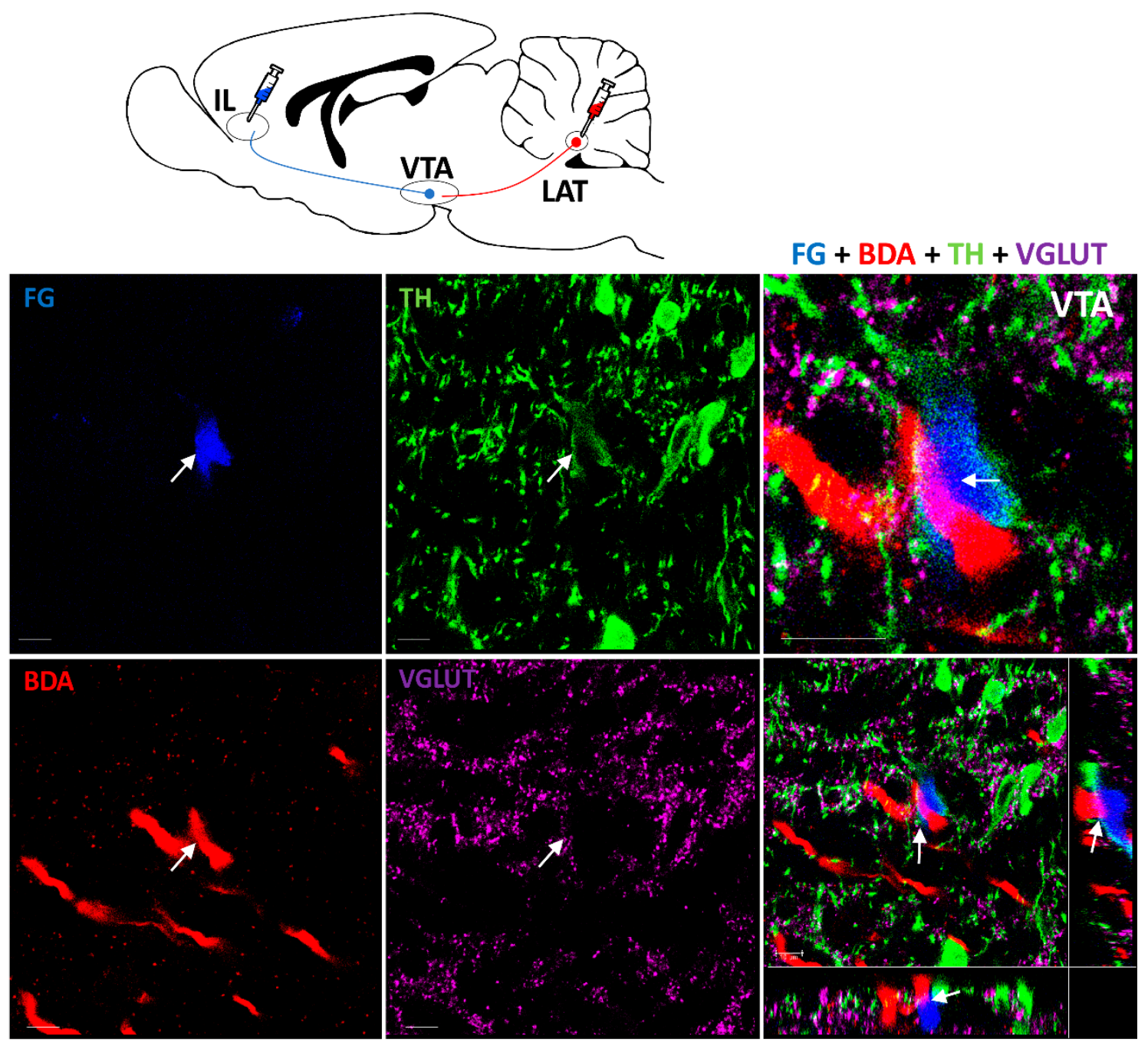

Figure 12. Coronal section of the VTA. Infusions of BDA (red) in the lateral nucleus and FG (blue) in the IL cortex. The arrows indicate an example of a synaptic contact between the terminal of a projection neuron from Lat nucleus (BDA+) and one TH+ neuron in the VTA (FG+). The dopamine neuron was identified as expressing tyrosine hydroxylase (TH/green). Vesicular glutamate transporter (vGluT2/purple). All confocal images were taken at $40 x$ with $2.5 x$ zoom. Scale bar of $10 \mu \mathrm{m}$. Right top panel: Digital amplification of $300 x$.

\section{DISCUSSION}

The present research is aimed at providing an explanatory model for the facilitative effect of a lesion in the apical region of lobule VIII on cocaine-induced conditioned memory. First, our findings showed that damage in this region of the vermis induced a general disinhibition in the MPFC and striatal subdivisions that receive dopaminergic projections mainly from the VTA. Second, they also showed that impairment of the posterior vermis induced an upregulation of PNN expression in the mPFC. Finally, we found a direct projection from the lateral nucleus to the VTA that also receives Purkinje 


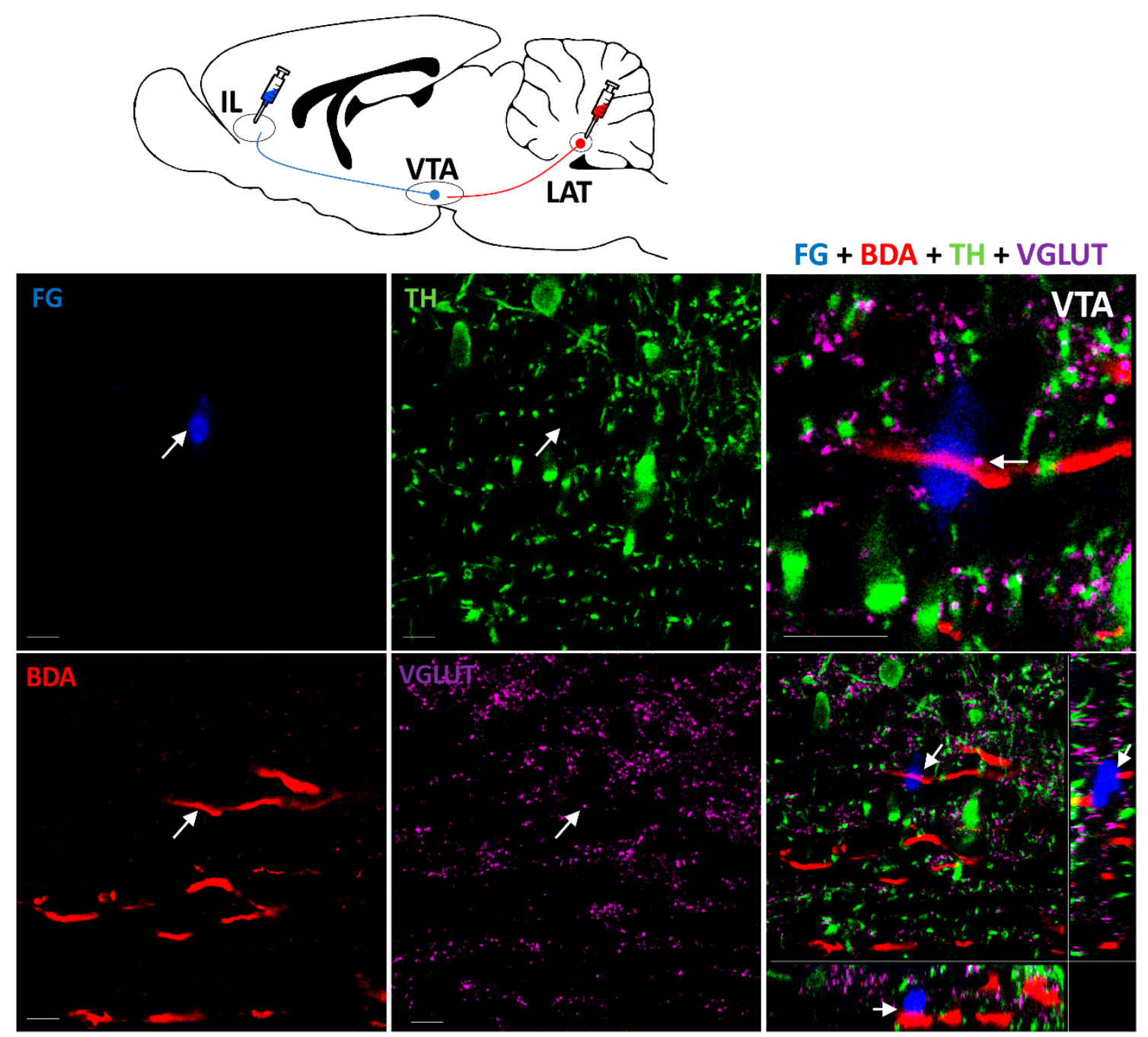

Figure 13. Coronal section of the VTA. Infusions of BDA (red) in the lateral nucleus and FG (blue) in the IL cortex. The arrows indicate an example of a synaptic contact between the terminal of a projection neuron from Lat nucleus (BDA+) and one TH+ neuron in the VTA $(F G+)$. Dopamine neuron was identified as expressing tyrosine hydroxylase (TH/green). Vesicular glutamate transporter (vGluT2/purple). All the pictures are taken a $40 x$ and $2.5 x$ zoom in a confocal. Scale bar of $10 \mu \mathrm{m}$. Digital co-labelling amplification of 300x.

axons from lobule VIII in the vermis. Hypothetically, this pathway might control activity and plasticity of the cortico-striatal circuitry through an increase in dopaminergic activity. The working hypothetical model that emerged from the present findings predicts that the impairment of the posterior cerebellar cortex, by increasing activity in the lateral nucleus would heighten glutamate release within the VTA and facilitate the release of DA in the $\mathrm{MPFC}$ and striatal regions.

The idea of a cerebellar modulation of the VTA is not new. It has been grounded in a few previous findings which described indirect cerebellar-VTA pathways (Forster and Blaha, 2003; Rogers et al, 2011), but also a direct control of the cerebellum onto the VTA (Carta 
et al, 2019; Watabe-Uchida et al, 2012). Indeed, the cerebellum could reach the VTA through the reticulotegmental and pedunculopontine nuclei (Forster and Blaha, 2003) and the mediodorsal and ventrolateral thalamus (Rogers et al, 2011). More importantly, there is a direct cerebellar-VTA pathway (Watabe-Uchida et al, 2012), whose functional properties have been recently delineated in an elegant study (Carta et al, 2019). Supporting the present working model, the optogenetic stimulation of the cerebellarVTA pathway increased firing in one third of VTA neurons in vivo, elicited excitatory synaptic currents, and induced strong place preference for the location in which mice received the optogenetic stimulation of the cerebellar projection (Carta et al, 2019). Moreover, the optogenetic stimulation of the cerebellar axons was as rewarding as the direct optogenetic activation of dopaminergic neurons in the VTA (Carta et al, 2019).

The fact that a small lesion in the posterior vermis was able to induce an upregulation of PNN expression in the MPFC was remarkable. PNNs have been proposed as one of the mechanisms for the stabilization of drug-induced memories (Sorg et al, 2016). Interestingly, previous findings demonstrated that drug-induced conditioned preference and drug self-administration increase PNN expression in the cerebellum (Carbo-Gas et al, 2017) and different prefrontal areas (Blacktop et al, 2017; VazquezSanroman et al, 2017). Furthermore, PNN digestion within the prelimbic cortex and the anterior lateral hypothalamus prevented the expression of cocaine-induced conditioned place preference (Slaker et al, 2015) and cocaine self-administration (Blacktop et al, 2017). The present data suggest that the cerebellar lesion by increasing synaptic stabilization mechanisms in the MPFC could facilitate the acquisition of cocaine-cue associations and strengthen drug-induced memories.

In our opinion, the major finding of the present research is to provide an explanatory model for the function of the posterior cerebellar vermis on drug-related memory. In this model (Figure 14), damage of the posterior vermis would release striatum-cortical networks from the inhibitory tonic control exerted by the cerebellum over the VTA, thereby promoting drug effects. The present findings may help to explain why patients with lesions or diseases affecting the posterior cerebellum presented difficulties in controlling their behaviour and emotions (Kim et al, 2013; Schmahmann and Sherman, 1998; Silveri et al, 1994; Tessier et al, 2015). Moreover, our model predicts that the 
stimulation of the posterior cerebellar vermis would reduce drug-related effects and improve behavioural inhibition.

In summary, the present results indicate that: (1) the posterior cerebellar cortex may exert an inhibitory control over the striatum and mPFC; $(2)$ the lateral nucleus seems to be the most plausible exit route for the cerebellar cortex to modulate activity and plasticity in the prefrontal-striatal network; and (3) the VTA could be a candidate to mediate cerebellar influences on activity and plasticity in prefrontal-striatal loops that in turn can regulate cocaine-related behaviour.
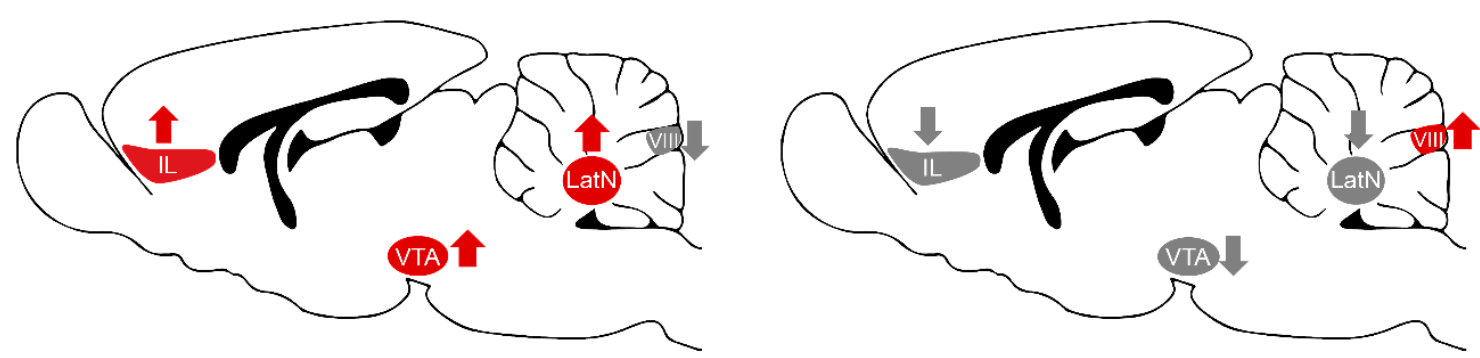

Figure 14. Working neuroanatomical model for the function of lobule VIII on drug-related memory. Left panel: The lesion of lobule VIII by increasing activity in the lateral nucleus can heighten glutamate release within the VTA and facilitate the release of DA in the MPFC and striatal regions. The lesion would release striatum-cortical networks from the inhibitory tonic control exerted by the cerebellum over the VTA, thereby promoting drug effects. Right panel: The model predicts that the stimulation of the posterior cerebellar vermis would reduce drug-related effects and improve behavioural inhibition.

\section{ACKNOWLEDGMENTS}

This research was supported by the following grants and fellowships: Ministerio de Educación Cultura y Deporte (FPU12/04059); Universitat Jaume I (PREDOC2014/11); UJI (14I307.01/1); Ministerio de Economía y Competitividad (MINECO) (PSI2015-68600-P); and Plan Nacional de Drogas 2017 (PND-132400).

\section{AUTHOR DISCLOSURE}

All authors declare no conflicts of interest. 


\section{AUTHORSHIP}

All authors made a notable contribution to the manuscript, and they were involved in critically revising the present version. Isis Gil-Miravet performed the stereotaxic surgeries and behavioural experiments; Isis Gil-Miravet and Francisco Olucha-Bordonau performed the tracing study; Isis Gil-Miravet, Edgar Arias de Saavedra-Sandoval, and Lizbeth Vásquez-Celaya were involved in image and data analysis. Finally, Marta Miquel designed the study, supervised the surgeries and behavioural experiments, was involved in data analysis, and drafted the manuscript. All authors approved the present version of the manuscript.

\section{DATA ACCESSIBILITY}

Raw data are available from the corresponding author upon request. 


\section{REFERENCES}

Anderson CM, Maas LC, Frederick B, Bendor JT, Spencer TJ, Livni E, et al (2006). Cerebellar Vermis Involvement in Cocaine-Related Behaviors. Neuropsychopharmacology 31: 1318-1326.

Ball GG, Micco DJ, Berntson GG (1974). Cerebellar stimulation in the rat: complex stimulation-bound oral behaviors and self-stimulation. Physiol Behav 13: 123-7.

Blacktop JM, Todd RP, Sorg BA (2017). Role of perineuronal nets in the anterior dorsal lateral hypothalamic area in the acquisition of cocaine-induced conditioned place preference and self-administration. Neuropharmacology 118: 124-136.

Bonson KR, Grant SJ, Contoreggi CS, Links JM, Metcalfe J, Weyl HL, et al (2002). Neural Systems and Cue-Induced Cocaine Craving. Neuropsychopharmacology 26: 376386.

Bostan AC, Dum RP, Strick PL (2018). Functional Anatomy of Basal Ganglia Circuits with the Cerebral Cortex and the Cerebellum. Prog Neurol Surg 33: 50-61.

Buckner RL, Krienen FM, Castellanos A, Diaz JC, Yeo BTT (2011). The organization of the human cerebellum estimated by intrinsic functional connectivity. J Neurophysiol 106: 2322-2345.

Callu D, Puget S, Faure A, Guegan M, Massioui N El (2007). Habit learning dissociation in rats with lesions to the vermis and the interpositus of the cerebellum. Neurobiol Dis 27: 228-237.

Carbo-Gas M, Moreno-Rius J, Guarque-Chabrera J, Vazquez-Sanroman D, Gil-Miravet I, Carulli $D$, et al (2017). Cerebellar perineuronal nets in cocaine-induced pavlovian memory: Site matters. Neuropharmacology 125: 166-180.

Carbo-Gas M, Vazquez-Sanroman D, Aguirre-Manzo L, Coria-Avila GA, Manzo J, SanchisSegura C, et al (2014a). Involving the cerebellum in cocaine-induced memory: Pattern of cFos expression in mice trained to acquire conditioned preference for cocaine. Addict Biol 19: 61-76.

Carbo-Gas M, Vazquez-Sanroman D, Gil-Miravet I, las Heras-Chanes J De, Coria-Avila GA, Manzo J, et al (2014b). Cerebellar hallmarks of conditioned preference for cocaine. Physiol Behav 132: 24-35.

Carta I, Chen CH, Schott AL, Dorizan S, Khodakhah K (2019). Cerebellar modulation of the reward circuitry and social behavior. Science (80- ) 364: .

Chen CH, Fremont R, Arteaga-Bracho EE, Khodakhah K (2014). Short latency cerebellar modulation of the basal ganglia. Nat Neurosci 17: 1767-1775.

Corbett D, Fox E, Milner PM (1982). Fiber pathways associated with cerebellar selfstimulation in the rat: a retrograde and anterograde tracing study. Behav Brain Res 6: 167-84.

Forster GL, Blaha CD (2003). Pedunculopontine tegmental stimulation evokes striatal 
dopamine efflux by activation of acetylcholine and glutamate receptors in the midbrain and pons of the rat. Eur J Neurosci 17: 751-762.

Fuentes P, Barrós-Loscertales A, Bustamante JC, Rosell P, Costumero V, Ávila C (2012). Individual differences in the Behavioral Inhibition System are associated with orbitofrontal cortex and precuneus gray matter volume. Cogn Affect Behav Neurosci 12: 491-8.

Geisler S, Zahm DS (2005). Afferents of the ventral tegmental area in the rat-anatomical substratum for integrative functions. J Comp Neurol 490: 270-294.

Gil-Miravet I, Guarque-Chabrera J, Carbo-Gas M, Olucha-Bordonau F, Miquel M (2018). The role of the cerebellum in drug-cue associative memory: functional interactions with the medial prefrontal cortex. Eur J Neurosci 1-10doi:10.1111/ejn.14187.

Grant S, London ED, Newlin DB, Villemagne VL, Liu X, Contoreggi C, et al (1996). Activation of memory circuits during cue-elicited cocaine craving. Proc Natl Acad Sci U S A 93: 12040-5.

Hoshi E, Tremblay L, Féger J, Carras PL, Strick PL (2005). The cerebellum communicates with the basal ganglia. Nat Neurosci 8: 1491-1493.

Ichinohe N, Mori F, Shoumura K (2000). A di-synaptic projection from the lateral cerebellar nucleus to the laterodorsal part of the striatum via the central lateral nucleus of the thalamus in the rat. Brain Res 880: 191-197.

Ikai Y, Takada M, Shinonaga Y, Mizuno N (1992). Dopaminergic and non-dopaminergic neurons in the ventral tegmental area of the rat project, respectively, to the cerebellar cortex and deep cerebellar nuclei. Neuroscience 51: 719-728.

Kasanetz F, Mato S, Sepers M, Inserm OJM, Cedex B (2008). Addiction and synaptic plasticity in the nucleus accumbens. Area 661: 269-296.

Kim JH, Kim TH, Choi YC, Chung S-C, Moon SW (2013). Impulsive behavior and recurrent major depression associated with dandy-walker variant. Psychiatry Investig 10: 303-5.

LaLumiere RT, Niehoff KE, Kalivas PW (2010). The infralimbic cortex regulates the consolidation of extinction after cocaine self-administration. Learn Mem 17: 168175.

Lalumiere RT, Smith KC, Kalivas PW (2012). Neural circuit competition in cocaineseeking: Roles of the infralimbic cortex and nucleus accumbens shell. Eur J Neurosci 35: 614-622.

Middleton FA, Strick PL (2000). Basal ganglia and cerebellar loops: Motor and cognitive circuits. Brain Res Rev 31: 236-250.

Middleton FA, Strick PL (2001). Cerebellar Projections to the Prefrontal Cortex of the Primate. J Neurosci 21: 700-712.

Miquel M, Toledo R, Garcia L, Coria-Avila G, Manzo J (2009). Why Should We Keep the Cerebellum in Mind When Thinking About Addiction? Curr Drug Abus Rev 2: 26-40. 
Miquel M, Vazquez-Sanroman D, Carbo-Gas M, Gil-Miravet I, Sanchis-Segura C, Carulli $D$, et al (2016). Have we been ignoring the elephant in the room? Seven arguments for considering the cerebellum as part of addiction circuitry. Neurosci Biobehav Rev 60: 1-11.

Moulton EA, Elman I, Becerra LR, Goldstein RZ, Borsook D (2014). The cerebellum and addiction: Insights gained from neuroimaging research. Addict Biol 19: 317-331.

Panagopoulos NT, Papadopoulos GC, Matsokis NA (1991). Dopaminergic innervation and binding in the rat cerebellum. Neurosci Lett 130: 208-212.

Paxinos G, Watson C (1998). The Rat Brain in Stereotaxic Coordinates. Acad Press 1474doi:10.1007/s13398-014-0173-7.2.

Rogers TD, Dickson PE, Heck DH, Goldowitz D, Mittleman G, Blaha CD (2011). Connecting the dots of the cerebro-cerebellar role in cognitive function: Neuronal pathways for cerebellar modulation of dopamine release in the prefrontal cortex. Synapse 65: 1204-1212.

Sacchetti B, Baldi E, Lorenzini CA, Bucherelli C (2002). Differential contribution of some cortical sites to the formation of memory traces supporting fear conditioning. Exp Brain Res 146: 223-232.

Sang L, Qin W, Liu Y, Han W, Zhang Y, Jiang T, et al (2012). Resting-state functional connectivity of the vermal and hemispheric subregions of the cerebellum with both the cerebral cortical networks and subcortical structures. Neuroimage 61: 12131225.

Schindelin J, Arganda-Carreras I, Frise E, Kaynig V, Longair M, Pietzsch T, et al (2012). Fiji: an open-source platform for biological-image analysis. Nat Methods 9: 676-82.

Schmahmann JD, Pandya DN (1997). Anatomic organization of the basilar pontine projections from prefrontal cortices in rhesus monkey. J Neurosci 17: 438-458.

Schmahmann JD, Sherman JC (1998). The cerebellar cognitive affective syndrome. Brain 121: 561-579.

Schneider F, Habel U, Wagner M, Franke P, Salloum JB, Shah NJ, et al (2001). Subcortical correlates of craving in recently abstinent alcoholic patients. Am J Psychiatry 158: 1075-1083.

Silveri MC, Leggio MG, Molinari M (1994). The cerebellum contributes to linguistic production: a case of agrammatic speech following a right cerebellar lesion. Neurology 44: 2047-50.

Slaker M, Churchill L, Todd RP, Blacktop JM, Zuloaga DG, Raber J, et al (2015). Removal of Perineuronal Nets in the Medial Prefrontal Cortex Impairs the Acquisition and Reconsolidation of a Cocaine-Induced Conditioned Place Preference Memory. J Neurosci 35: 4190-4202.

Sorg BA, Berretta S, Blacktop JM, Fawcett JW, Kitagawa H, Kwok JCF, et al (2016). Casting a Wide Net: Role of Perineuronal Nets in Neural Plasticity. J Neurosci 36: 1145911468. 
Stanton GB (1980). Topographical organization of ascending cerebellar projections from the dentate and interposed nuclei in Macaca mulatta: An anterograde degeneration study. J Comp Neurol 190: 699-731.

Tessier A, Cosin C, Mayo W, Pfeuty M, Misdrahi D, Sibon I (2015). Impulsive aggressive obsessions following cerebellar strokes: a case study. J Neurol 262: 1775-1776.

Turner BM, Paradiso S, Marvel CL, Pierson R, Boles Ponto LL, Hichwa RD, et al (2007). The cerebellum and emotional experience. Neuropsychologia 45: 1331-1341.

Vazquez-Sanroman D, Carbo-Gas M, Leto K, Cerezo-Garcia M, Gil-Miravet I, SanchisSegura C, et al (2015a). Cocaine-induced plasticity in the cerebellum of sensitised mice. Psychopharmacology (Berl) 232: 4455-4467.

Vazquez-Sanroman D, Leto K, Cerezo-Garcia M, Carbo-Gas M, Sanchis-Segura C, Carulli $D$, et al (2015b). The cerebellum on cocaine: Plasticity and metaplasticity. Addict Biol 20: 941-955.

Vazquez-Sanroman DB, Monje RD, Bardo MT (2017). Nicotine self-administration remodels perineuronal nets in ventral tegmental area and orbitofrontal cortex in adult male rats. Addict Biol 22: 1743-1755.

Wagner MJ, Kim TH, Savall J, Schnitzer MJ, Luo L (2017). Cerebellar granule cells encode the expectation of reward. Nature 544: 96-100.

Watabe-Uchida M, Zhu L, Ogawa SK, Vamanrao A, Uchida N (2012). Whole-Brain Mapping of Direct Inputs to Midbrain Dopamine Neurons. Neuron 74: 858-873.

Watson TC, Becker N, Apps R, Jones MW (2014). Back to front: cerebellar connections and interactions with the prefrontal cortex. Front Syst Neurosci 8: 4.

Witter L, Rudolph S, Pressler RT, Lahlaf SI, Regehr WG (2016). Purkinje Cell Collaterals Enable Output Signals from the Cerebellar Cortex to Feed Back to Purkinje Cells and Interneurons. Neuron 91: 312-319.

Xiao L, Bornmann C, Hatstatt-Burklé L, Scheiffele P (2018). Regulation of striatal cells and goal-directed behavior by cerebellar outputs. Nat Commun 9: .

Yalachkov Y, Kaiser J, Naumer MJ (2010). Sensory and motor aspects of addiction. Behav Brain Res 207: 215-222.

Zhu L, Sacco T, Strata P, Sacchetti B (2011). Basolateral amygdala inactivation impairs learning-induced long-term potentiation in the cerebellar cortex. PLoS One 6: e16673. 
GENERAL DISCUSSION 



\section{GENERAL DISCUSSION}

The present research focused on providing an explanatory model of the cerebellum's role in cocaine-induced conditioned memory. As we have already said, addiction is a neuroplasticity disorder that involves mechanisms similar to those proposed for learning and memory (Hyman et al, 2006; Miles et al, 2003; Milton and Everitt, 2012). It is well established that the MPFC is involved in reward learning and addiction (Tzschentke and Schmidt, 2000). Several studies have observed that excitotoxic lesions of mPFC enhance cocaine-seeking and facilitate cocaine self-administration by increasing sensitivity to effects of cocaine reinforcement (Schenk et al, 1991; Weissenborn et al, 1997), which may be due to a behavioural disinhibition after mPFC impairment. On the contrary, the implication of the cerebellum in addiction is very recent. A large number of studies have demonstrated the involvement of the cerebellum in memory, decision-making, emotional processing, and executive functions (Sacchetti et al, 2002b; Schmahmann and Pandya, 1997; Turner et al, 2007; Watson et al, 2014; Zhu et al, 2011), all of them affected in addicted subjects. Moreover, reciprocal loops between the cerebellum and the prefrontal-striatal-limbic circuity have been clearly demonstrated (Bostan et al, 2018; Buckner et al, 2011; Chen et al, 2014; Hoshi et al, 2005; Ichinohe et al, 2000; Ikai et al, 1992; Middleton and Strick, 2000, 2001; Panagopoulos et al, 1991; Sang et al, 2012; Stanton, 1980; Xiao et al, 2018). Taken together, the background information underpin the hypothesis of a significant cerebellar role in learning, memory, and addiction.

The present thesis proposes a functional relationship between the IL and the dorsal area of lobe VIII. Impairment of either of these regions increases the percentage of animals that developed a preference for the cues associated with cocaine. In both cases, the deactivation affected the number of animals expressing cocaine-induced conditioned preference, but not the magnitude of the conditioned response that was very similar to the sham individuals expressing conditioned preference. Behavioural similarities were also found when we deactivated the PL cortex or the ventral region of lobule VIII. However, the functional relationship between these areas could not be explored in depth and thereby we have not been able to establish any hypothesis about their functions and relationships, beyond the behavioural effects described in the first chapter. 
To propose a hypothetical working model that can explain the effects of brain and cerebellar deactivations, we then explored changes in neuronal activity and plasticity as an effect of deactivations. The results of mPFC deactivations were consistent with those already published in the previous literature. As previous studies have shown, IL and PL have opposite functions in drug-seeking (Ball and Slane, 2012; Capriles et al, 2003; McFarland and Kalivas, 2001; Ongür and Price, 2000; Peters et al, 2009; Pfarr et al, 2015; Sierra-Mercado et al, 2011). In the present thesis, we observed that the inactivation of the IL cortex, in addition to promoting cocaine-induced memory, also increased activity in the posterior cerebellum and NAcSh. Similar results were observed with respect to the intensity of PNNs, which were more intense and stronger in lobule VIII and IX from all animals expressing preference, regardless of the IL deactivation. These findings suggest that the cerebellar activity and plasticity modifications are not directly related to the IL impairment but the development of preference. Thus, the increase in cerebellar activity would be linked to the reactivation of previous acquired drug-cue association in order to select between CS+ and CS-. In fact, when the behavioural selection was prevented as occurs when the animal is confined in the CS+ compartment, cerebellar activation was not seen (Carbo-Gas et al, 2017). Moreover, stronger PNNs around Golgi interneurons in the posterior vermis might promote a stabilization of the new connections representing cocaine-related memories. Contrary to what was observed after the IL deactivation, PL impairment during conditioning induced a similar expression of cFos and PNNs to that of the groups that did not show preference. Importantly, mPFC deactivations did not have effects when rats were trained under a random association between the cue and drug. Consequently, these animals did not develop cocaineinduced conditioned preference, and their cFos and PNN expression did not differ from the no preference groups. These data argue in favour of memory-related effects on cerebellar activity and plasticity after mPFC deactivations.

Worthy of mention, the expression of cocaine-induced preference was associated with stronger PNNs around Golgi cells in the dorsal cerebellar cortex, supporting previous studies in mice (Carbo-Gas et al, 2017). Golgi cells are inhibitory interneurons (Eccles et al, 1964) that crucially control the temporal dynamics and the spatial distribution of information through the cerebellar cortex (D'Angelo, 2009). Golgi cells regulate the 
synchronization of granule cell activity, as well as spike timing and burst transmission, determining the sign, intensity, and duration of long-term synaptic plasticity at the mossy fibre-granule cell relay (D’Angelo, 2009; Eccles et al, 1964). Palay and Chan-Palay (1974) showed that Golgi cells receive glutamatergic inputs from granule cells and mossy fibres within the cerebellar glomerulus (Palay and Chan-Palay, 1974). Conversely, Golgi cells receive GABAergic and glycinergic inhibitory signals from stellate, basket, and Lugaro cells (Sotelo and Llinás, 1972). Thus, Golgi cells are essential in the control of synaptic plasticity at the level of the granular layer. Here, we described that cerebellardependent mechanisms of cocaine associative memory are involved in the upregulation of PNNs around Golgi cells. The majority of Golgi neurons that express stronger PNNs are active (Carbo-Gas et al, 2017). It has been shown that blocking inhibition of Golgi cells turns the balance in favour of long-term potentiation in the cerebellar cortex (Mapelli and D'Angelo, 2007). On the contrary, when inhibition is higher than excitation, long-term depression may dominate ( $D^{\prime}$ Angelo and De Zeeuw, 2009). Therefore, one can speculate that the establishment of cocaine memory may encourage the feedforward inhibitory control by Golgi cells.

A neurotoxic lesion of the dorsal cerebellar cortex in lobule VIII generated a global disinhibition in prefrontal and striatal regions, as well as stronger PNNs around GABAergic interneurons of the mPFC. Previous research demonstrated that the degradation of PNNs in the PL cortex blocked conditioned place preference (Slaker et al, 2015) and prevented reinstatement to drug-seeking (Slaker et al, 2018). Our findings also suggest that the lateral nucleus is the most likely cerebellar output to mediate the present effects. Both activity and PNN expression increased specifically in this DCN after the lesion. No significant lesion effects were seen on the other DCN. Remarkably, as the tracing study demonstrated, the dorsal region of lobule VIII in the vermis sends projections to all of the DCN, including the most lateral portions. However, when infusing the retrograde tracer into the VTA, we only were able to find colocalization in the interpositus and lateral nuclei, but not in the medial nucleus. More importantly, the most caudal region of the contralateral VTA (the parabrachial pigmented nucleus/PBP) receives direct projections from the lateral nucleus that seem to be glutamatergic. Retrograde tracing from the IL cortex indicated that cerebellum-VTA projections make 
synaptic contacts with dopaminergic neurons that reach the IL cortex. Altogether, the present findings strongly claim in favour of a key role of the cerebellum in the establishment of drug-cue associations (Miquel et al, 2009, 2016) and point to the caudal VTA as the interface through which the cerebellum would regulate the activity and plasticity of the prefrontal-striatal loops. Our results support previous studies that also found direct projections from the DCN to VTA (Carta et al, 2019; Watabe-Uchida et $a l, 2012)$. The role of the VTA and dopaminergic projections to the prefrontal cortex in reward and drug addiction are well established (Chao and Nestler, 2004; Geisler and Zahm, 2005). However, only very recently, it has been shown that optogenetic stimulation of cerebellar projections activate VTA neurons and induce powerful rewarding effects (Carta et al, 2019).

The resulting hypothetical neuroanatomical model indicates that the posterior cerebellar cortex may exert a direct inhibitory modulation of the prefrontal-striatal circuitry controlling dopaminergic activity of the VTA neurons. Under impairment of the posterior cerebellar cortex, the lateral nucleus, by increasing glutamatergic activity release within the VTA, encourages activity of dopaminergic neurons within the MPFC and striatal regions.

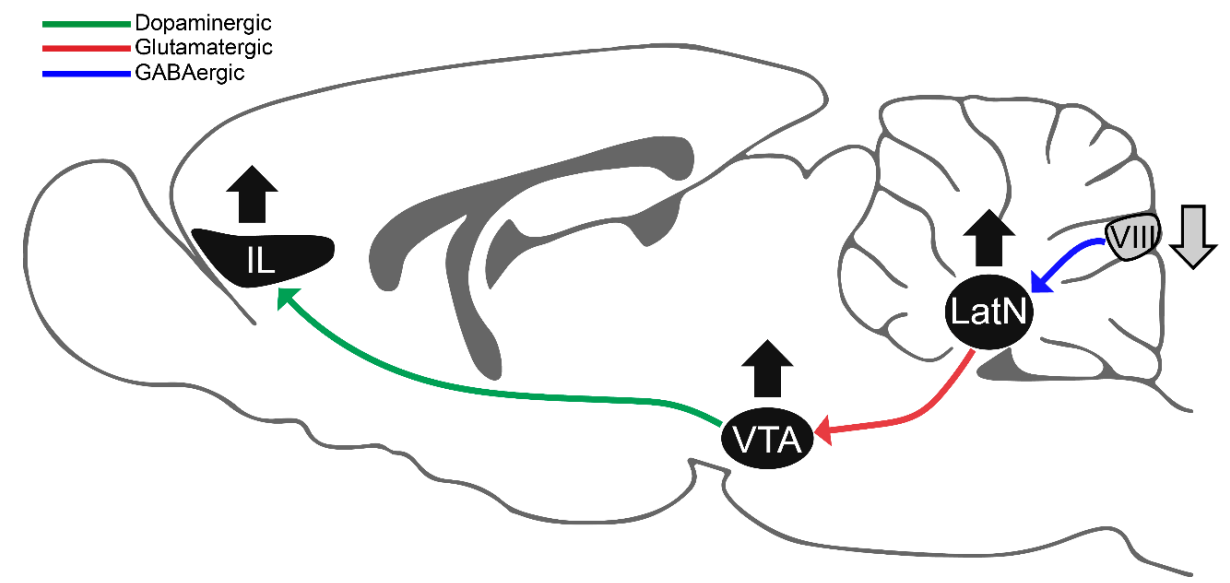

In our view, this hypothetical model has a high predictive power. Specifically, the cerebellum may be crucial for restraining ongoing actions when environmental conditions change by adjusting prefrontal activity in response to the new external and internal stimuli, thereby promoting flexible behavioural control. Beyond the regulation of cocaine-induced preference conditioning, our model may explain why cerebellar lesions have been shown to generate behavioural disinhibition, impulsivity, and compulsivity (Miquel et al., in press). 
STRENGTHS AND PITFALLS 



\section{STRENGTHS AND PITFALLS}

\section{Strengths}

- The main contribution of this thesis is to propose a working neuroanatomical model for the role of the cerebellum in drug addiction with a high predictive power. Moreover, this work represents the first experimental approach to explain the modulatory function of the posterior cerebellum on the prefrontal-striatal circuitry for the establishment of cocaine-associated memories.

- Another significant contribution is the demonstration of a direct cerebellar control over the VTA.

- This thesis also provides support for a functional relationship between the cerebellum and the medial prefrontal cortex in drug-related memory.

\section{Pitfalls, weaknesses, and future directions}

- The difficulties to maintain cannulas in the cerebellum forced us to perform permanent injuries in this region. This approach generated a difference between the animals with mPFC deactivations and the animals with lesions in the cerebellum on the test day. In future research, both regions should be studied under the same conditions during the acquisition and expression phases.

- The tracer study shows connection networks between the cerebellum and the prefrontal cortex. It would be interesting to study further the outputs of neurons from the cerebellum, as well as the targeted neurons in the VTA.

- The direct pathway Lateral nucleus-VTA should be blocked and stimulated to confirm the model.

- It would also be interesting to use DREADDs in future research to activate and deactivate all these regions and be able to alter the entire circuit at certain times.

- Finally, beyond the establishment of cocaine-induced Pavlovian memory, it would be interesting to observe how our deactivations affect other paradigms of selfadministration, as well as models of extinction and reconsolidation. 
REFERENCES 



\section{REFERENCES}

Adamaszek M, D’Agata F, Ferrucci R, Habas C, Keulen S, Kirkby KC, et al (2017). Consensus Paper: Cerebellum and Emotion. Cerebellum 16: 552-576.

Ahmed SH (2012). The science of making drug-addicted animals. Neuroscience 211: 107125.

Albus JS (1971). A theory of cerebellar function. Math Biosci 10: 25-61.

Andersen BB, Korbo L, Pakkenberg B (1992). A quantitative study of the human cerebellum with unbiased stereological techniques. J Comp Neurol 326: 549-560.

Anderson CM, Maas LC, Frederick B, Bendor JT, Spencer TJ, Livni E, et al (2006). Cerebellar Vermis Involvement in Cocaine-Related Behaviors. Neuropsychopharmacology 31: 1318-1326.

Apps R, Garwicz M (2005). Anatomical and physiological foundations of cerebellar information processing. Nat Rev Neurosci 6: 297-311.

Ball KT, Slane M (2012). Differential involvement of prelimbic and infralimbic medial prefrontal cortex in discrete cue-induced reinstatement of 3,4methylenedioxymethamphetamine (MDMA; ecstasy) seeking in rats. Psychopharmacology (Berl) 224: 377-85.

Barbour B, Häusser M (1997). Intersynaptic diffusion of neurotransmitter. Trends Neurosci 20: 377-384.

Barros-Loscertales A, Garavan H, Bustamante JC, Ventura-Campos N, Llopis JJ, Belloch $V$, et al (2011). Reduced striatal volume in cocaine-dependent patients. Neuroimage 56: 1021-1026.

Belin D, Balado E, Piazza PV, Deroche-Gamonet V (2009). Pattern of Intake and Drug Craving Predict the Development of Cocaine Addiction-like Behavior in Rats. Biol Psychiatry 65: 863-868.

Belin D, Everitt BJ (2008). Cocaine seeking habits depend upon dopamine-dependent serial connectivity linking the ventral with the dorsal striatum. Neuron 57: 432-441.

Blacktop JM, Todd RP, Sorg BA (2017). Role of perineuronal nets in the anterior dorsal lateral hypothalamic area in the acquisition of cocaine-induced conditioned place preference and self-administration. Neuropharmacology 118: 124-136.

Blackwood N, Ffytche D, Simmons A, Bentall R, Murray R, Howard R (2004). The cerebellum and decision making under uncertainty. Cogn Brain Res 20: 46-53.

Boele HJ, Koekkoek SKE, Zeeuw Cl De (2010). Cerebellar and extracerebellar involvement in mouse eyeblink conditioning: the ACDC model. Front Cell Neurosci 3: 19.

Bonson KR, Grant SJ, Contoreggi CS, Links JM, Metcalfe J, Weyl HL, et al (2002). Neural Systems and Cue-Induced Cocaine Craving. Neuropsychopharmacology 26: 376386. 
Bossert JM, Stern a L, Theberge FR, Cifani C, Koya E, Hope BT, et al (2011). Ventral medial prefrontal cortex neuronal ensembles mediate context-induced relapse to heroin. Nat Neurosci 14: 420-422.

Bostan AC, Dum RP, Strick PL (2010). The basal ganglia communicate with the cerebellum. Proc Natl Acad Sci 107: 8452-8456.

Bostan AC, Dum RP, Strick PL (2013). Cerebellar networks with the cerebral cortex and basal ganglia. Trends Cogn Sci 17: 241-254.

Bostan AC, Dum RP, Strick PL (2018). Functional Anatomy of Basal Ganglia Circuits with the Cerebral Cortex and the Cerebellum. Prog Neurol Surg 33: 50-61.

Bostan AC, Strick PL (2010). The cerebellum and basal ganglia are interconnected. Neuropsychol Rev 20: 261-270.

Bostan AC, Strick PL (2018). The basal ganglia and the cerebellum: nodes in an integrated network. Nat Rev Neurosci 19: 338-350.

Broche-Pérez Y, Herrera Jiménez LF, Omar-Martínez E (2016). Bases neurales de la toma de decisiones. Neurologia 31: 319-325.

Brodal P (Oxford University Press: New York, 2016). The Central Nervous System. doi:10.1093/med/9780190228958.001.0001.

Brückner G, Brauer K, Härtig W, Wolff JR, Rickmann MJ, Derouiche A, et al (1993). Perineuronal nets provide a polyanionic, glia-associated form of microenvironment around certain neurons in many parts of the rat brain. Glia 8: 183-200.

Brunel N, Hakim V, Isope P, Nadal JP, Barbour B (2004). Optimal information storage and the distribution of synaptic weights: Perceptron versus Purkinje cell. Neuron 43: 745-757.

Buckner RL, Krienen FM, Castellanos A, Diaz JC, Yeo BTT (2011). The organization of the human cerebellum estimated by intrinsic functional connectivity. J Neurophysiol 106: 2322-2345.

Capriles N, Rodaros D, Sorge RE, Stewart J (2003). A role for the prefrontal cortex in stress- and cocaine-induced reinstatement of cocaine seeking in rats. Psychopharmacology (Berl) 168: 66-74.

Carballo-Márquez A, Vale-Martínez A, Guillazo-Blanch G, Martí-Nicolovius M (2009). Muscarinic receptor blockade in ventral hippocampus and prelimbic cortex impairs memory for socially transmitted food preference. Hippocampus 19: 446-455.

Carbo-Gas M, Moreno-Rius J, Guarque-Chabrera J, Vazquez-Sanroman D, Gil-Miravet I, Carulli D, et al (2017). Cerebellar perineuronal nets in cocaine-induced pavlovian memory: Site matters. Neuropharmacology 125: 166-180.

Carbo-Gas M, Vazquez-Sanroman D, Aguirre-Manzo L, Coria-Avila GA, Manzo J, SanchisSegura C, et al (2014a). Involving the cerebellum in cocaine-induced memory: Pattern of cFos expression in mice trained to acquire conditioned preference for cocaine. Addict Biol 19: 61-76. 
Carbo-Gas M, Vazquez-Sanroman D, Gil-Miravet I, las Heras-Chanes J De, Coria-Avila GA, Manzo J, et al (2014b). Cerebellar hallmarks of conditioned preference for cocaine. Physiol Behav 132: 24-35.

Cardinal RN, Parkinson JA, Hall J, Everitt BJ (2002). Emotion and motivation: the role of the amygdala, ventral striatum, and prefrontal cortex. Neurosci Biobehav Rev 26: 321-52.

Carta I, Chen CH, Schott AL, Dorizan S, Khodakhah K (2019). Cerebellar modulation of the reward circuitry and social behavior. Science (80- ) 364: .

Carulli D, Rhodes KE, Brown DJ, Bonnert TP, Pollack SJ, Oliver K, et al (2006). Composition of perineuronal nets in the adult rat cerebellum and the cellular origin of their components. J Comp Neurol 494: 559-577.

Chao J, Nestler EJ (2004). Molecular neurobiology of drug addiction. Annu Rev Med 55: 113-132.

Chen $\mathrm{CH}$, Fremont R, Arteaga-Bracho EE, Khodakhah K (2014). Short latency cerebellar modulation of the basal ganglia. Nat Neurosci 17: 1767-1775.

Courchesne E, Allen $\mathrm{G}$ (1997). Prediction and preparation, fundamental functions of the cerebellum. Learn Mem 4: 1-35.

Crombag HS, Shaham Y (2002). Renewal of drug seeking by contextual cues after prolonged extinction in rats. Behav Neurosci 116: 169-73.

D'Angelo E (2009). The critical role of Golgi cells in regulating spatio-temporal integration and plasticity at the cerebellum input stage. Front Neurosci 2: 35-46.

D’Angelo E, Zeeuw Cl De (2009). Timing and plasticity in the cerebellum: focus on the granular layer. Trends Neurosci 32: 30-40.

Doya K (2000). Complementary roles of basal ganglia and cerebellum in learning and motor control. Curr Opin Neurobiol 10: 732-739.

Eccles J, Llinás R, Sasaki K (1964). Golgi Cell Inhibition in the Cerebellar Cortex. Nature 204: 1265-1266.

Everitt BJ, Robbins TW (2005). Neural systems of reinforcement for drug addiction: from actions to habits to compulsion. Nat Neurosci 8: 1481-1489.

Forster GL, Blaha CD (2003). Pedunculopontine tegmental stimulation evokes striatal dopamine efflux by activation of acetylcholine and glutamate receptors in the midbrain and pons of the rat. Eur J Neurosci 17: 751-762.

Foscarin S, Ponchione D, Pajaj E, Leto K, Gawlak M, Wilczynski GM, et al (2011). Experience-dependent plasticity and modulation of growth regulatory molecules at central synapses. PLoS One 6: e16666.

Fuchs RA, Evans KA, Ledford CC, Parker MP, Case JM, Mehta RH, et al (2005). The role of the dorsomedial prefrontal cortex, basolateral amygdala, and dorsal hippocampus in contextual reinstatement of cocaine seeking in rats. Neuropsychopharmacology 30: 296-309. 
Gallinat J, Meisenzahl E, Jacobsen LK, Kalus P, Bierbrauer J, Kienast T, et al (2006). Smoking and structural brain deficits: A volumetric MR investigation. Eur J Neurosci 24: 1744-1750.

Gao Z, Proietti-Onori M, Lin Z, Brinke MM ten, Boele HJ, Potters JW, et al (2016). Excitatory Cerebellar Nucleocortical Circuit Provides Internal Amplification during Associative Conditioning. Neuron 89: 645-657.

Geisler S, Zahm DS (2005). Afferents of the ventral tegmental area in the rat-anatomical substratum for integrative functions. J Comp Neurol 490: 270-294.

Gilbert PFC, Thach WT (1977). Purkinje cell activity during motor learning. Brain Res 128: 309-328.

Gogolla N, Caroni P, Lüthi A, Herry C (2009). Perineuronal nets protect fear memories from erasure. Science (80- ) 325: 1258-1261.

Goldstein RZ, Volkow ND (2002). Drug addiction and its underlying neurobiological basis: neuroimaging evidnce for the involvement of the frontal cortex. Am J Psychiatry 159: 1642-1652.

Goldstein RZ, Volkow ND (2011). Dysfunction of the prefrontal cortex in addiction: Neuroimaging findings and clinical implications. Nat Rev Neurosci 12: 652-669.

Goto Y, Grace AA (2005). Dopaminergic modulation of limbic and cortical drive of nucleus accumbens in goal-directed behavior. Nat Neurosci 8: 805-12.

Grant S, London ED, Newlin DB, Villemagne VL, Liu X, Contoreggi C, et al (1996). Activation of memory circuits during cue-elicited cocaine craving. Proc Natl Acad Sci U S A 93: 12040-5.

Habas C, Kamdar N, Nguyen D, Prater K, Beckmann CF, Menon V, et al (2009). Distinct cerebellar contributions to intrinsic connectivity networks. J Neurosci 29: 85868594.

Haber SN, Fudge JL, McFarland NR (2000). Striatonigrostriatal Pathways in Primates Form an Ascending Spiral from the Shell to the Dorsolateral Striatum. J Neurosci 20: 2369-2382.

Haines DE, Dietrichs E (2011). The cerebellum - structure and connections. Handb Clin Neurol 103: 3-36.

Hitchcott PK, Phillips GD (1997). Amygdala and hippocampus control dissociable aspects of drug-associated conditioned rewards. Psychopharmacology (Berl) 131: 187-195.

Hoshi E, Tremblay L, Féger J, Carras PL, Strick PL (2005). The cerebellum communicates with the basal ganglia. Nat Neurosci 8: 1491-1493.

Hyman SE, Malenka RC, Nestler EJ (2006). Neural mechanisms of addiction: the role of reward-related learning and memory. Annu Rev Neurosci 29: 565-98.

Ichinohe N, Mori F, Shoumura K (2000). A di-synaptic projection from the lateral cerebellar nucleus to the laterodorsal part of the striatum via the central lateral nucleus of the thalamus in the rat. Brain Res 880: 191-197. 
Ikai $\mathrm{Y}$, Takada $\mathrm{M}$, Mizuno $\mathrm{N}$ (1994). Single neurons in the ventral tegmental area that project to both the cerebral and cerebellar cortical areas by way of axon collaterals. Neuroscience 61: 925-934.

Ikai Y, Takada M, Shinonaga Y, Mizuno N (1992). Dopaminergic and non-dopaminergic neurons in the ventral tegmental area of the rat project, respectively, to the cerebellar cortex and deep cerebellar nuclei. Neuroscience 51: 719-728.

Ito M (1984). The modifiable neuronal network of the cerebellum. Jpn J Physiol 34: 78192.

Jiménez-Rivera CA, Segarra O, Jiménez Z, Waterhouse BD (2000). Effects of intravenous cocaine administration on cerebellar Purkinje cell activity. Eur J Pharmacol 407: 91100.

Kalivas P (2004). Glutamate systems in cocaine addiction. Pharmacology 4: 23-29.

Kalivas PW, McFarland K (2003). Brain circuitry and the reinstatement of cocaineseeking behavior. Psychopharmacology (Berl) 168: 44-56.

Kalivas PW, Volkow ND (2005). The neural basis of addiction: A pathology of motivation and choice. Am J Psychiatry 162: 1403-1413.

Klitenick MA, Tham C-S, Fibiger HC (1995). Cocaine and d-amphetamine increase c-fos expression in the rat cerebellum. Synapse 19: 29-36.

Li Q, Li W, Wang H, Wang Y, Zhang Y, Zhu J, et al (2015). Predicting subsequent relapse by drug-related cue-induced brain activation in heroin addiction: An event-related functional magnetic resonance imaging study. Addict Biol 20: 968-978.

Lin WC, Chou KH, Chen HL, Huang CC, Lu CH, Li SH, et al (2012). Structural deficits in the emotion circuit and cerebellum are associated with depression, anxiety and cognitive dysfunction in methadone maintenance patients: A voxel-based morphometric study. Psychiatry Res - Neuroimaging 201: 89-97.

Mapelli J, D'Angelo E (2007). The Spatial Organization of Long-Term Synaptic Plasticity at the Input Stage of Cerebellum. J Neurosci 27: 1285-1296.

Mariën $\mathrm{P}$, Ackermann $\mathrm{H}$, Adamaszek $\mathrm{M}$, Barwood CHS, Beaton A, Desmond J, et al (2014). Consensus paper: Language and the cerebellum: An ongoing enigma. Cerebellum 13: 386-410.

Marr D (1969). A theory of cerebellar cortex. J Physiol 202: 437-70.

Matus-Amat P, Higgins EA, Sprunger D, Wright-Hardesty K, Rudy JW (2007). The Role of Dorsal Hippocampus and Basolateral Amygdala NMDA Receptors in the Acquisition and Retrieval of Context and Contextual Fear Memories. Behav Neurosci 121: 721731.

McFarland K, Kalivas PW (2001). The circuitry mediating cocaine-induced reinstatement of drug-seeking behavior. J Neurosci 21: 8655-8663.

McFarland K, Lapish CC, Kalivas PW (2003). Prefrontal Glutamate Release into the Core ofthe Nucleus Accumbens Mediates Cocaine-Induced Reinstatement of Drug- 
Seeking Behavior. J Neurosci 23: 3531-3537.

Middleton FA, Strick PL (2000). Basal ganglia and cerebellar loops: Motor and cognitive circuits. Brain Res Rev 31: 236-250.

Middleton FA, Strick PL (2001). Cerebellar Projections to the Prefrontal Cortex of the Primate. J Neurosci 21: 700-712.

Miles FJ, Everitt BJ, Dickinson A (2003). Oral cocaine seeking by rats: action or habit? Behav Neurosci 117: 927-938.

Milton AL, Everitt BJ (2012). The persistence of maladaptive memory: Addiction, drug memories and anti-relapse treatments. Neurosci Biobehav Rev 36: 1119-1139.

Miquel M, Toledo R, Garcia L, Coria-Avila G, Manzo J (2009). Why Should We Keep the Cerebellum in Mind When Thinking About Addiction? Curr Drug Abus Rev 2: 26-40.

Miquel M, Vazquez-Sanroman D, Carbo-Gas M, Gil-Miravet I, Sanchis-Segura C, Carulli $D$, et al (2016). Have we been ignoring the elephant in the room? Seven arguments for considering the cerebellum as part of addiction circuitry. Neurosci Biobehav Rev 60: 1-11.

Moers-Hornikx VMP, Sesia T, Basar K, Lim LW, Hoogland G, Steinbusch HWM, et al (2009). Cerebellar nuclei are involved in impulsive behaviour. Behav Brain Res 203: 256-263.

Morales AM, Lee B, Hellemann G, O'Neill J, London ED (2012). Gray-matter volume in methamphetamine dependence: Cigarette smoking and changes with abstinence from methamphetamine. Drug Alcohol Depend 125: 230-238.

Moulton EA, Elman I, Becerra LR, Goldstein RZ, Borsook D (2014). The cerebellum and addiction: Insights gained from neuroimaging research. Addict Biol 19: 317-331.

O'Doherty JP, Dayan P, Friston K, Critchley H, Dolan RJ (2003). Temporal difference models and reward-related learning in the human brain. Neuron 38: 329-37.

Oever MC Van den, Spijker S, Smit AB, Vries TJ De (2010). Prefrontal cortex plasticity mechanisms in drug seeking and relapse. Neurosci Biobehav Rev 35: 276-284.

Ongür D, Price JL (2000). The organization of networks within the orbital and medial prefrontal cortex of rats, monkeys and humans. Cereb cortex 10: 206-219.

Ostlund SB, Balleine BW (2005). Lesions of Medial Prefrontal Cortex Disrupt the Acquisition But Not the Expression of Goal-Directed Learning. J Neurosci 25: 77637770.

Palay SL, Chan-Palay V (1974). Cerebellar Cortex: Cytology and Organization. New York, NY Springer-Verlag doi:10.1016/0022-510X(76)90245-8.

Panagopoulos NT, Papadopoulos GC, Matsokis NA (1991). Dopaminergic innervation and binding in the rat cerebellum. Neurosci Lett 130: 208-212.

Pelloux Y, Everitt BJ, Dickinson A (2007). Compulsive drug seeking by rats under punishment: Effects of drug taking history. Psychopharmacology (Berl) 194: 127137. 
Pelloux Y, Murray JE, Everitt BJ (2013). Differential roles of the prefrontal cortical subregions and basolateral amygdala in compulsive cocaine seeking and relapse after voluntary abstinence in rats. Eur J Neurosci 38: 3018-3026.

Peters J, Kalivas PW, Quirk GJ (2009). Extinction circuits for fear and addiction overlap in prefrontal cortex. Learn Mem 16: 279-288.

Pfarr S, Meinhardt MW, Klee ML, Hansson AC, Vengeliene V, Schonig K, et al (2015). Losing Control: Excessive Alcohol Seeking after Selective Inactivation of CueResponsive Neurons in the Infralimbic Cortex. J Neurosci 35: 10750-10761.

Phillips RG, LeDoux JE (1992). Differential Contribution of Amygdala and Hippocampus to Cued and Contextual Fear Conditioning. Behav Neurosci 106: 274-285.

Robinson TE, Berridge KC (2003). Addiction. Annu Rev Psychol 54: 25-53.

Rogers TD, Dickson PE, Heck DH, Goldowitz D, Mittleman G, Blaha CD (2011). Connecting the dots of the cerebro-cerebellar role in cognitive function: Neuronal pathways for cerebellar modulation of dopamine release in the prefrontal cortex. Synapse 65: 1204-1212.

Romberg C, Yang S, Melani R, Andrews MR, Horner AE, Spillantini MG, et al (2013). Depletion of Perineuronal Nets Enhances Recognition Memory and Long-Term Depression in the Perirhinal Cortex. J Neurosci 33: 7057-7065.

Sacchetti B, Baldi E, Lorenzini CA, Bucherelli C (2002a). Cerebellar role in fearconditioning consolidation. Proc Natl Acad Sci U S A 99: 8406-8411.

Sacchetti B, Baldi E, Lorenzini CA, Bucherelli C (2002b). Differential contribution of some cortical sites to the formation of memory traces supporting fear conditioning. Exp Brain Res 146: 223-232.

Sacchetti B, Scelfo B, Strata P (2005). The cerebellum: Synaptic changes and fear conditioning. Neuroscientist 11: 217-227.

Sacchetti B, Scelfo B, Tempia F, Strata P (2004). Long-term synaptic changes induced in the cerebellar cortex by fear conditioning. Neuron 42: 973-982.

Sang L, Qin W, Liu Y, Han W, Zhang Y, Jiang T, et al (2012). Resting-state functional connectivity of the vermal and hemispheric subregions of the cerebellum with both the cerebral cortical networks and subcortical structures. Neuroimage 61: 12131225.

Schenk S, Horger BA, Peltier R, Shelton K (1991). Supersensitivity to the reinforcing effects of cocaine following 6-hydroxydopamine lesions to the medial prefrontal cortex in rats. Brain Res 543: 227-35.

Schmahmann JD (1991). An Emerging Concept: The Cerebellar Contribution to Higher Function. Arch Neurol 48: 1178-1187.

Schmahmann JD, Pandya DN (1997). The cerebrocerebellar system. IntRevNeurobiol 41: 31-60.

Schmahmann JD, Sherman JC (1998). The cerebellar cognitive affective syndrome. Brain 
121: $561-579$.

Schneider F, Habel U, Wagner M, Franke P, Salloum JB, Shah NJ, et al (2001). Subcortical correlates of craving in recently abstinent alcoholic patients. Am J Psychiatry 158: 1075-1083.

Schoenbaum G, Setlow B, Saddoris MP, Gallagher M (2003). Encoding predicted outcome and acquired value in orbitofrontal cortex during cue sampling depends upon input from basolateral amygdala. Neuron 39: 855-67.

Schweighofer N, Doya K, Kuroda S (2004). Cerebellar aminergic neuromodulation: towards a functional understanding. Brain Res Brain Res Rev 44: 103-16.

See RE, Kruzich PJ, Grimm JW (2001). Dopamine, but not glutamate, receptor blockade in the basolateral amygdala attenuates conditioned reward in a rat model of relapse to cocaine-seeking behavior. Psychopharmacology (Berl) 154: 301-10.

Shaham Y, Shalev U, Lu L, Wit H de, Stewart J (2003). The reinstatement model of drug relapse: history, methodology and major findings. Psychopharmacology (Berl) 168: 3-20.

Shear PK, Sullivan E V, Lane B, Pfefferbaum A (1996). Mammillary body and cerebellar shrinkage in chronic alcoholics with and without amnesia. Alcohol Clin Exp Res 20: 1489-1495.

Shinoda Y, Sugihara I, Wu HS, Sugiuchi Y (2000). The entire trajectory of single climbing and mossy fibers in the cerebellar nuclei and cortex. Prog Brain Res 124: 173-86.

Sierra-Mercado D, Padilla-Coreano N, Quirk GJ (2011). Dissociable roles of prelimbic and infralimbic cortices, ventral hippocampus, and basolateral amygdala in the expression and extinction of conditioned fear. Neuropsychopharmacology 36: 529538.

Sim ME, Lyoo IK, Streeter CC, Covell J, Sarid-Segal O, Ciraulo DA, et al (2007). Cerebellar Gray Matter Volume Correlates with Duration of Cocaine Use in CocaineDependent Subjects. Neuropsychopharmacology 32: 2229-2237.

Slaker M, Blacktop JM, Sorg BA (2016). Caught in the net: Perineuronal nets and addiction. Neural Plast 2016: .

Slaker M, Churchill L, Todd RP, Blacktop JM, Zuloaga DG, Raber J, et al (2015). Removal of Perineuronal Nets in the Medial Prefrontal Cortex Impairs the Acquisition and Reconsolidation of a Cocaine-Induced Conditioned Place Preference Memory. J Neurosci 35: 4190-4202.

Slaker ML, Jorgensen ET, Hegarty DM, Liu X, Kong Y, Zhang F, et al (2018). Cocaine Exposure Modulates Perineuronal Nets and Synaptic Excitability of Fast-Spiking Interneurons in the Medial Prefrontal Cortex. Eneuro 5: .

Smith KS, Graybiel AM (2013). A dual operator view of habitual behavior reflecting cortical and striatal dynamics. Neuron 79: 361-374.

Sorg BA, Berretta S, Blacktop JM, Fawcett JW, Kitagawa H, Kwok JCF, et al (2016). Casting a Wide Net: Role of Perineuronal Nets in Neural Plasticity. J Neurosci 36: 11459- 
11468.

Sotelo C, Llinás R (1972). Specialized membrane junctions between neurons in the vertebrate cerebellar cortex. J Cell Biol 53: 271-289.

Stanton GB (1980). Topographical organization of ascending cerebellar projections from the dentate and interposed nuclei in Macaca mulatta: An anterograde degeneration study. J Comp Neurol 190: 699-731.

Stefanik MT, Kalivas PW (2013). Optogenetic dissection of basolateral amygdala projections during cue-induced reinstatement of cocaine seeking. Front Behav Neurosci 7: 213.

Strata P, Scelfo B, Sacchetti B (2011). Involvement of cerebellum in emotional behavior. Physiol Res 60: S39-S48.

Thompson RF, Steinmetz JE (2009). The role of the cerebellum in classical conditioning of discrete behavioral responses. Neuroscience 162: 732-755.

Timmann D, Drepper J, Frings M, Maschke M, Richter S, Gerwig M, et al (2010). The human cerebellum contributes to motor, emotional and cognitive associative learning. A review. Cortex 46: 845-857.

Tsien RY (2013). Very long-term memories may be stored in the pattern of holes in the perineuronal net. Proc Natl Acad Sci 110: 12456-12461.

Turner BM, Paradiso S, Marvel CL, Pierson R, Boles Ponto LL, Hichwa RD, et al (2007). The cerebellum and emotional experience. Neuropsychologia 45: 1331-1341.

Tzschentke TM, Schmidt WJ (2000). Differential effects of discrete subarea-specific lesions of the rat medial prefrontal cortex on amphetamine- and cocaine-induced behavioural sensitization. Cereb cortex 10: 488-498.

Upadhyay J, Maleki N, Potter J, Elman I, Rudrauf D, Knudsen J, et al (2010). Alterations in brain structure and functional connectivity in prescription opioid-dependent patients. Brain 133: 2098-2114.

Vazquez-Sanroman D, Carbo-Gas M, Leto K, Cerezo-Garcia M, Gil-Miravet I, SanchisSegura C, et al (2015a). Cocaine-induced plasticity in the cerebellum of sensitised mice. Psychopharmacology (Berl) 232: 4455-4467.

Vazquez-Sanroman D, Leto K, Cerezo-Garcia M, Carbo-Gas M, Sanchis-Segura C, Carulli $D$, et al (2015b). The cerebellum on cocaine: Plasticity and metaplasticity. Addict Biol 20: 941-955.

Volkow ND, Wang GJ, Fowler JS, Tomasi D, Telang F, Baler R (2010). Addiction: Decreased reward sensitivity and increased expectation sensitivity conspire to overwhelm the brain's control circuit. BioEssays 32: 748-755.

Volkow ND, Wang GJ, Tomasi D, Baler RD (2013). Unbalanced neuronal circuits in addiction. Curr Opin Neurobiol 23: 639-648.

Voogd J, Glickstein M (1998). The anatomy of the cerebellum. Trends Neurosci 21: 370375. 
Wagner MJ, Kim TH, Savall J, Schnitzer MJ, Luo L (2017). Cerebellar granule cells encode the expectation of reward. Nature 544: 96-100.

Watabe-Uchida M, Zhu L, Ogawa SK, Vamanrao A, Uchida N (2012). Whole-Brain Mapping of Direct Inputs to Midbrain Dopamine Neurons. Neuron 74: 858-873.

Watson TC, Becker N, Apps R, Jones MW (2014). Back to front: cerebellar connections and interactions with the prefrontal cortex. Front Syst Neurosci 8: 4.

Weissenborn R, Robbins TW, Everitt BJ (1997). Effects of medial prefrontal or anterior cingulate cortex lesions on responding for cocaine under fixed-ratio and secondorder schedules of reinforcement in rats. Psychopharmacology (Berl) 134: 242-257.

White JJ, Sillitoe R V. (2013). Development of the cerebellum: From gene expression patterns to circuit maps. Wiley Interdiscip Rev Dev Biol 2: 149-164.

Wise RA (2009). Roles for nigrostriatal--not just mesocorticolimbic--dopamine in reward and addiction. Trends Neurosci 32: 517-24.

Xiao L, Bornmann C, Hatstatt-Burklé L, Scheiffele P (2018). Regulation of striatal cells and goal-directed behavior by cerebellar outputs. Nat Commun 9: .

Xue Y-X, Xue L-F, Liu J-F, He J, Deng J-H, Sun S-C, et al (2014). Depletion of Perineuronal Nets in the Amygdala to Enhance the Erasure of Drug Memories. J Neurosci 34: 6647-6658.

Yalachkov Y, Kaiser J, Naumer MJ (2010). Sensory and motor aspects of addiction. Behav Brain Res 207: 215-222.

Yamaguchi M, Suzuki T, Abe S, Hori T, Kurita H, Asada T, et al (2002). Repeated cocaine administration differentially affects NMDA receptor subunit (NR1, NR2A-C) mRNAs in rat brain. Synapse 46: 157-169.

Yin HH, Ostlund SB, Knowlton BJ, Balleine BW (2005). The role of the dorsomedial striatum in instrumental conditioning. Eur J Neurosci 22: 513-523.

Yoon B, Kim JS, Lee KS, Kim BS, Chung SR, Kim YI (2006). Early pathological changes in the cerebellum of patients with pure cerebellar syndrome demonstrated by diffusion-tensor imaging. Eur Neurol 56: 166-171.

Zhu L, Sacco T, Strata P, Sacchetti B (2011). Basolateral amygdala inactivation impairs learning-induced long-term potentiation in the cerebellar cortex. PLoS One 6: e16673. 
ACKNOWLEDGEMENTS 



\section{ACKNOWLEDGEMENTS}

Suena "Tie a Yellow Ribbon" de Brotherhood of Man mientras me siento frente al ordenador. La verdad es que me vienen a la cabeza miles de momentos e historias que me sacan una buena sonrisa. En estos 8 años han pasado por mi vida personas maravillosas que han hecho posible que esta tesis sea una realidad. Por esta razón hay muchas personas a las que tengo que agradecer este resultado.

Las primeras personas a las que tengo que agradecerles que todo esto haya sido posible es a mis padres. Sin ellos esos más de 3 años sin financiación hubieran sido irrealizables. Ellos me han dado la oportunidad de poder estar escribiendo ahora mismo estas palabras. Gracias por haberme enseñado a no rendirme ante las dificultades, a trabajar el doble para alcanzar los objetivos, a ser fuerte y pelear por mis sueños, y a levantarme siempre, aunque todo parezca hundirse. Gracias a mis hermanas por haber sido un pilar fundamental en mi vida, por haber llegado a este mundo para darle luz a mis momentos oscuros, por enseñarme a reírme de las dificultades, y que en la vida los problemas tienen únicamente el valor que tú quieras darle. Gracias a mis iaios por enseñarme los verdaderos valores de la vida, que luchar no es una opción sino un deber y que con trabajo duro se puede alcanzar todo, y es que poder seguir aprendiendo de ellos es el mejor regalo del mundo.

Aida gracias por los ánimos, el apoyo y por haber estado a mi lado durante todo este tiempo. Fuiste mi principal apoyo durante los primeros años, y aunque nunca llegaste a entender cómo podía trabajar por amor al arte, siempre has estado y sigues estando ahí cuando te necesito.

Majo gracias por estar ahí en los problemas y las alegrías, por los consejos, por las ganas de vivir, por no dejarme nunca tirar la toalla, por hacer de mi estancia algo más llevadero y haberme sacado sonrisas cuando nadie más lo conseguía, y sobre todo, por hacerme ver constantemente que yo podía con esto y con todo.

Gracias Silvia por estar siempre ahí y escucharme atentamente, aunque muchas veces no entendieras nada de lo que te decía. Siempre me has dado buenos consejos. 
Gracias a mi "gente guay" que siempre me dieron fuerzas para continuar y a todas esas quedadas, conversaciones y risas que me llevaron siempre arriba.

Gracias Ana por ser la sonrisa eterna con la que compartir esta lucha. Para mi eres un referente de trabajo y esfuerzo. Gracias por todos tus consejos acompañados siempre de una buena copa de vino. Tu arte me inspira.

Además de familia y amigos, también han ido y venido unas cuantas personas por el laboratorio que me han enseñado y aportado infinitas experiencias. Algunas de estas personas han terminado formando parte de mi vida y siendo mucho más que simples compañeros de trabajo. A todos ellos tengo que agradecerles gran parte del resultado.

A Marta por haber confiado en mí durante estos años y haberme ofrecido su apoyo en momentos difíciles. A parte de jefa, también has sido confidente y medio madre (eso me decías en los primeros congresos), y aunque somos dos personas de carácter fuerte que han chocado en múltiples ocasiones, siempre lo hemos solucionado y hemos vivido momentos únicos llenos de emoción, como cuando por fin recibí la beca, y compartido grandes experiencias. La sinceridad creo que ha sido el pilar central que ha sustentado siempre esta relación, y aunque el final ha sido crítico y duro para mí, la experiencia de estos 8 años ha sido maravillosa y enriquecedora. Gracias por haberme enseñado tanto.

A Paco por haberme enseñado tanto $y$ haber hecho del final de esta tesis algo maravilloso. Siempre has conseguido contagiarme tu alegría y tu ilusión, y no puedes hacerte a la idea de cuantas veces me has hecho sonreír. Gracias por los ánimos, los consejos y todo el cariño que me has dado.

A Mercè por toda su ayuda durante este proceso y haber tenido siempre unas palabras de ánimo o un buen consejo que ofrecer en los momentos oportunos. A Laura Font por todo su apoyo, sus consejos y todas las recetas que hemos compartido, y por supuesto, por ser mi follower no 1. A Raúl por sus ánimos y compartir sus experiencias pasadas. A Carla Sanchis por tener siempre un buen consejo que ofrecer y una forma distinta de ver la vida. A Ali y Sandra por los ánimos, las risas y toda la ayuda que siempre me han ofrecido en el animalario y fuera de él.

A Laura López por ser amiga y compañera. Confidente de lo bueno y de lo malo. Por todos los consejos y las veces que me transmitió calma. Por sus imitaciones que me 
hicieron reír hasta caer al suelo, y sobre todo, gracias por este empujón final que tanto necesitaba.

A Maria Carbó por enseñarme tantas cosas que han sido fundamentales para esta tesis y todos esos grandes momentos repletos de risas, como aquel primer congreso de Oviedo que nunca olvidaré, y es que al final los buenos momentos son los que se quedan.

A Noemí por todo su apoyo durante los primeros años y demostrarme que no es necesario seguir la corriente para labrarse un futuro, que subir una gran cuesta no significa tener que mantener el camino.

A Carla por todas sus refranes y frases sin sentido, porque reírse siempre es la mejor forma de empezar o terminar el día. Gracias por los ánimos, la energía y los conejos que siempre hemos compartido.

A Regu por su valentía y enseñarme el valor de ser uno mismo, de perseguir tus sueños y nunca conformarse con menos.

A Julián por el apoyo y el soporte que me ofreció en tantos momentos.

A Aitor por ser la persona más noble y pura que he conocido nunca. Amigo, hacen falta más personas como tú en este mundo. Gracias por enseñarme la bondad de tus actos y por todo tu ánimo, tu apoyo incondicional, tu fuerza y tu energía. Tú me has hecho ser mejor persona.

A Patri por enseñarme que enfrentarse a la vida a veces es complicado, pero siempre se puede resurgir con más fuerza y energía para alcanzar nuevos objetivos.

A Andrea por compartir tantas veces mi filosofía de vida, esas conversaciones de balcón infinitas y fluir a mi lado. Reírse de los problemas a tu lado siempre ha sido fácil. Trape, botella y fandango siempre serán una super pandi.

A María Palau por su buen rollo y saber transmitir siempre alegría y calma en todas las situaciones de la vida.

A Olga por toda su energía y entusiasmo, y es que cuando las fuerzas flaquean a veces necesitas personas que te contagien toda su energía positiva. 
A Nacho por sus ánimos, consejos y ser un luchador nato, y es que no importa quien intente ponerte trabas en el camino que tú siempre ves el lado bueno de todo y nadie consigue pararte.

A Edgar por su apoyo y sus locuras que siempre han conseguido sacarme una sonrisa justo en el momento adecuado.

A Liz por su bondad y su gran corazón, y por enseñarme que las buenas amistades pueden estar separadas por miles de kilómetros. Gracias por compartir todas tus experiencias conmigo y por escuchar todas mis tonterías. Tus consejos siempre han sido fortuna.

A Simona simplemente por ser tú. Gracias por estar ahí en estos últimos meses, por aguantar mis subidas y bajadas, por reírte conmigo de lo bueno y de lo malo, porque sin ti esta última etapa no hubiera sido lo mismo y es que a veces la vida nos pone a la persona adecuada en el momento oportuno. Me has llenado de calma y amor cuando más lo necesitaba. Gracias por haber llegado a mi vida.

Gracias por las cenas, los congresos, los rescates gatunos endemoniados, los jueves de láser, los cumpleaños, los abrazos, los besos y todos los buenos momentos. Podría escribir páginas y páginas de momentos inolvidables que me hacen sonreír cada vez que vuelven a mi mente y es que no puedo sentirme más afortunada de este largo viaje.

Gracias a todos.

Finalmente, solo me queda agradecerle a la Isis del pasado, la que no se rindió, la que nunca tiró la toalla. La que vivió una experiencia única en USA e intento sacarle el lado bueno a la situación. La que nunca renunció a sus valores. La que peleó cada una de las batallas e injusticias que se cruzaron en su camino. La que nunca dejo que nadie le pasara por encima. La que rio a carcajadas y disfruto de la gente que tenía a su alrededor. La que ofreció todo lo que sabía y aprendió todo lo que pudo. La que intentó hacer las cosas lo mejor posible, y aunque cometió muchos errores, siempre intentó aprender de ellos. Gracias. 